Aus der Abteilung Unfallchirurgie, Plastische und Wiederherstellungschirurgie

(Prof. Dr. med. K. M. Stürmer)

im Zentrum Chirurgie

der Medizinischen Fakultät der Universität Göttingen

Femorale und tibiale Transplantatfixation der vorderen Kreuzbandplastik mit der neuartigen bioresorbierbaren Interferenzschraube Milagro ${ }^{\oplus}$

Klinische und radiologische Ergebnisse nach einem Jahr

\author{
INAUGURAL - DISSERTATION \\ zur Erlangung des Doktorgrades \\ der Medizinischen Fakultät \\ der Georg-August-Universität zu Göttingen
}

vorgelegt von

Tobias Sawallich

aus

Albstadt-Ebingen

Göttingen 2010 
Dekan: Prof. Dr. med. C. Frömmel

I. Berichterstatter: Prof. Dr.med. K.-H. Frosch

II. Berichterstatter: Priv.-Doz. Dr. med. Baums

III. Berichterstatter/in: Prof. Dr.med., Dr. rer. nat. Crozier

Tag der mündlichen Prüfung: 9.2.2011 


\section{Inhaltsverzeichnis}

1. Einleitung ............................................................. 4

1.1 Biomechanik des humanen Kniegelenks...................................... 4

1.2 Anatomie und Funktion des vorderen Kreuzbandes............................ 5

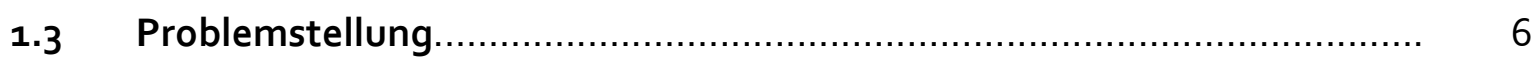

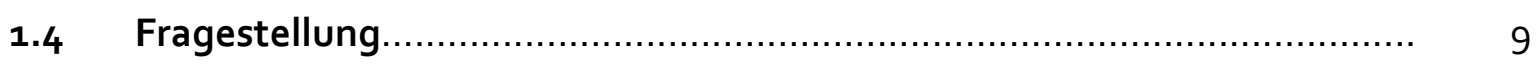

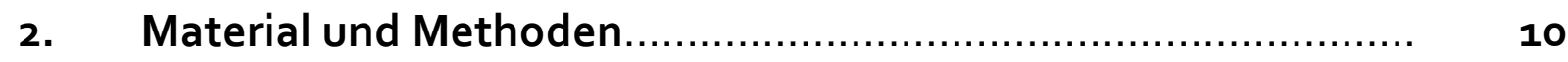

$2.1 \quad$ Patientenkollektiv und Ausschlusskriterien.................................... 10

2.2 Verletzungsursachen, Zeit zwischen Unfall und Operation.................... 10

2.3 Milagro ${ }^{\circledR}$-Interferenzschraube..................................................... 11

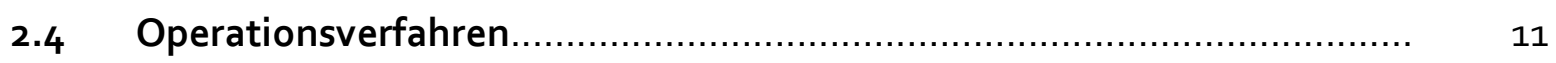

2.4.1 Diagnostische Arthroskopie .................................................. 11

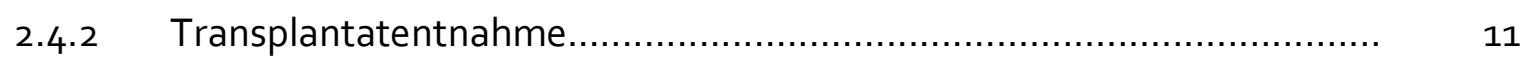

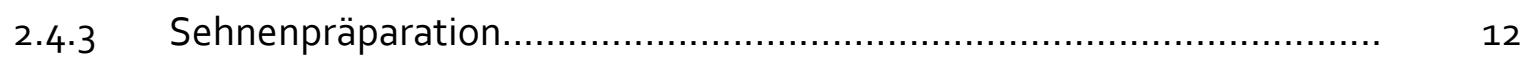

2.4.4 Anlage der Bohrkanäle ...................................................... 12

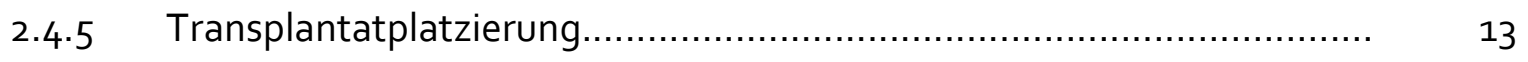

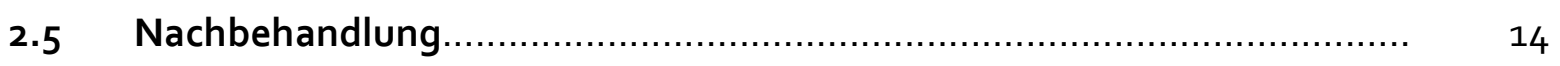

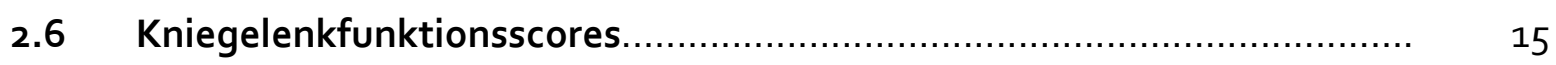

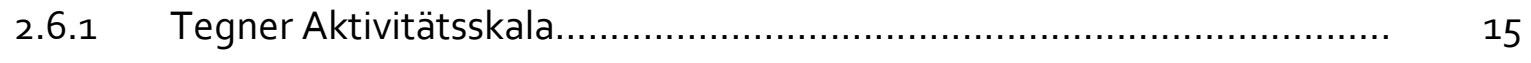

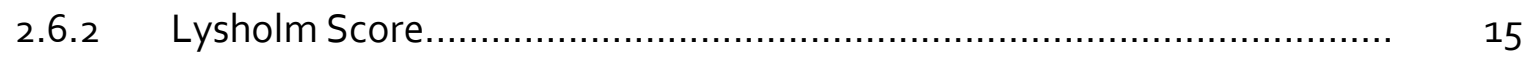

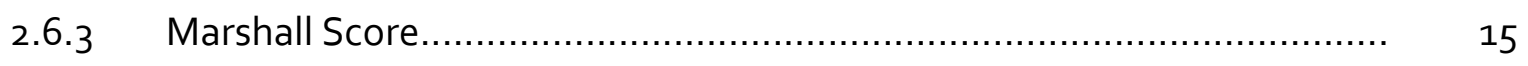

2.6.4 Visuelle Analogskala Schmerz und Funktion ..................................... 16

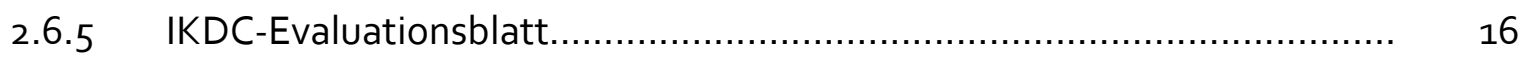

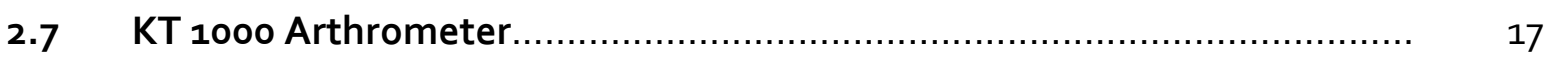

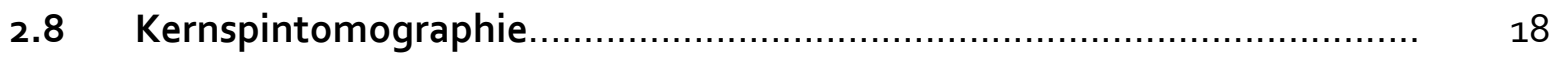

2.8.1 Methodik und Durchführung der Messung...................................... 18

2.8.2 Evaluation der Messgenauigkeit................................................... 19

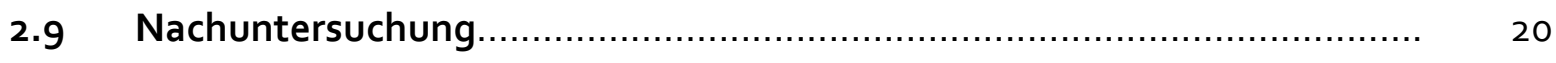

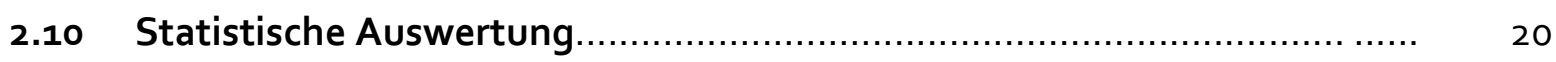




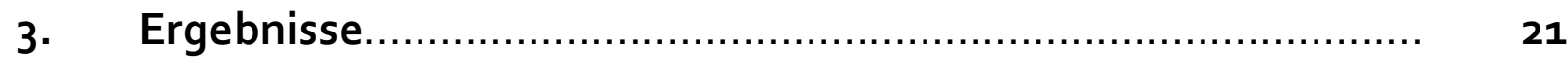

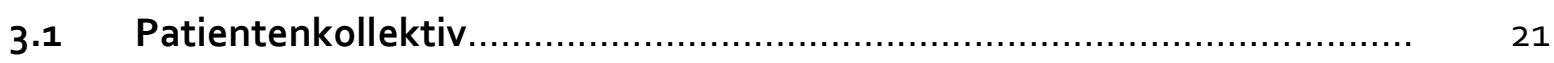

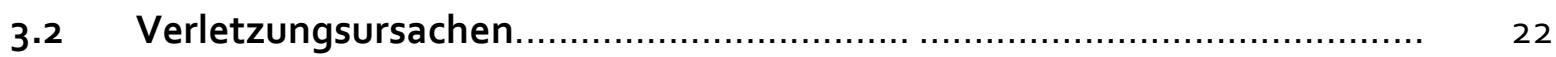

3.3 Klinische Ergebnisse bei Transplantatfixation mit Milagro ${ }^{\circledR}$ -

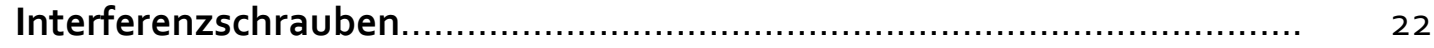

3.3.1 Tegner Aktivitätsskala.......................................................... 22

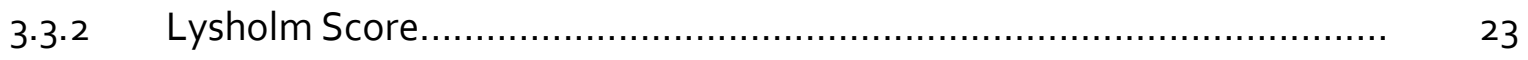

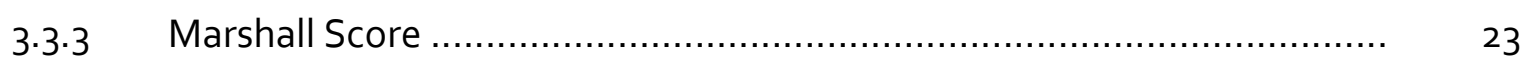

3.3.4 Visuelle Analogskala Schmerz und Funktion...................................... 24

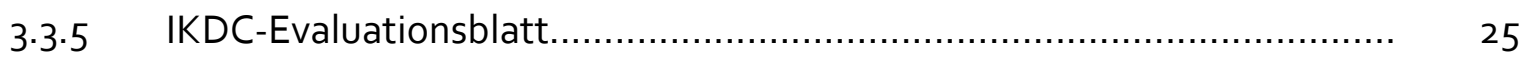

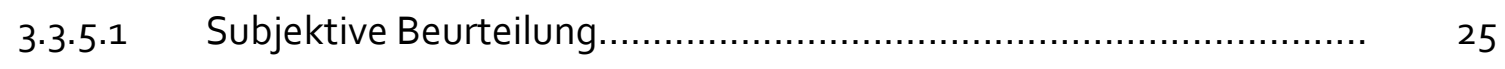

3.3.5.2 Rate optimaler Operationsergebnisse ...................................... 25

3.3.5.3 Objektive Beurteilung........................................................ 25

3.3.5.4 Symptome an der Transplantatentnahmestelle........................... 26

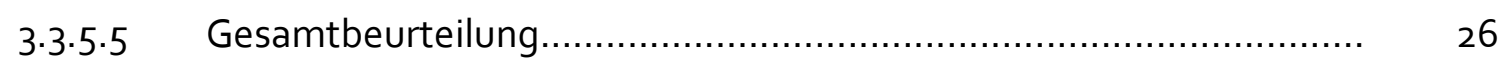

3.4 Vergleich des klinischen Ergebnisses bei VKB-Rekonstruktion mit der

\section{Semitendinosussehne alleine versus Semitendinosus- und}

Gracilissehne als Transplantat............................................... 27

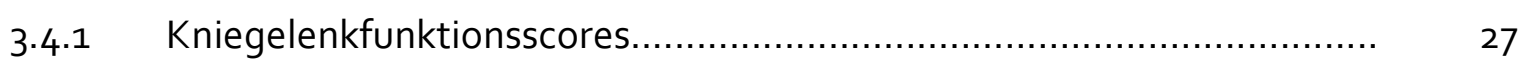

3.4.2 Instrumentelle Stabilitätsprüfung mit dem KT 1000 Arthrometer.......... 28

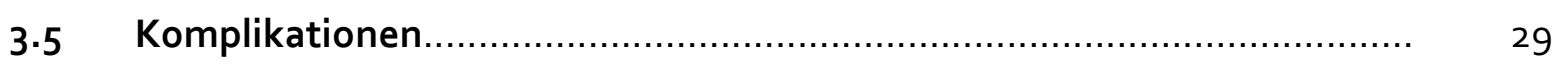

3.6 Milagro ${ }^{\circledR}$-Interferenzschrauben im konventionellen Röntgen................ 29

3.7 Kernspintomographische Ergebnisse .......................................... 30

3.7.1 Messgenauigkeit in der 3D-DESS Sequenz................................... 30

3.7.2 Milagro ${ }^{\circledR}$-Interferenzschraube in der Kernspintomographie.................... 30

3.7.3 Resorption der Milagro ${ }^{\circledR}$-Interferenzschrauben ................................. 31

3.7.3.1 Tibiale Milagro ${ }^{\circledR}$-Interferenzschraube............................................ 32

3.7.3.2 Femorale Milagro ${ }^{\circledR}$-Interferenzschraube....................................... 33

3.7.4 Tunnelenlargement bei Fixation mit Milagro ${ }^{\circledR}$-Interferenzschrauben... $\quad 34$

3.7.4.1 Tunnelenlargement des tibialen Bohrkanals................................ 36

3.7.4.2 Tunnelenlargement des femoralen Bohrkanals............................. 37

3.8 Ödeme, Knochenzysten und Osteolysen.......................................... 
4. Diskussion .................................................................. 39

4.1 Resorption der Milagro ${ }^{\circledR}$-Interferenzschraube .......................................... 39

4.2 Tunnelenlargement bei Fixation mit der Milagro ${ }^{\circledR}$. Interferenzschraube ....................................................................... 41

4.3 Klinische Ergebnisse bei Transplantatfixation mit Milagro ${ }^{\circledR}$ Interferenzschrauben ............................................................ 43

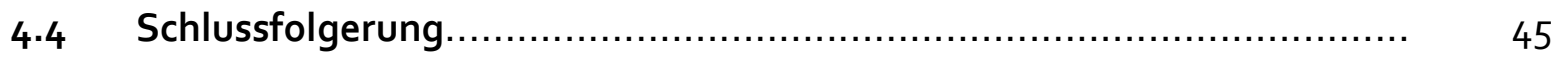

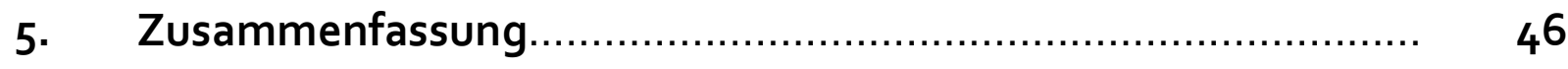

6. Literaturverzeichnis...................................................... $\quad 48$

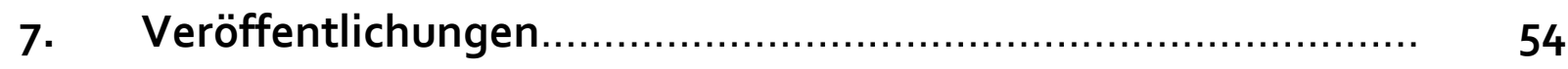

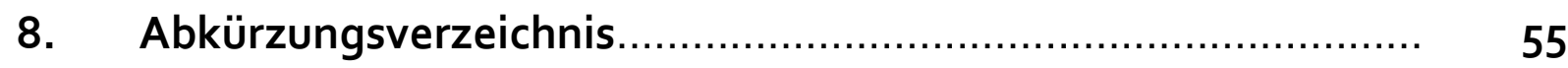

9. Abbildungs- und Tabellenverzeichnis................................. 57

10. Anhang: Scores............................................................ 5 


\section{Einleitung}

\subsection{Biomechanik des humanen Kniegelenks}

Das Kniegelenk besteht aus einem femoropatellaren und einem tibiofemoralen Gelenk (TFG). Im TFG artikulieren die nicht kongruenten Gelenkflächen der Femurkondylen mit dem flachen Tibiaplateau. Schon im Jahre 1836 fanden die Gebrüder Weber aus Göttingen heraus, dass der Bewegungsablauf im TFG durch eine Roll-Gleitbewegung gekennzeichnet ist (Weber 1836). Fischer hat durch Realanalyse der Röntgenserienbilder von Zuppinger präzisiert, dass bei Flexion des Kniegelenks initial die Gelenkflächen abrollen und ab einem Flexionswinkel von etwa 20-30 aufeinander Gleiten (Fischer 1907, Zuppinger 1904).

Auf dem Tibiaplateau legen die Femurkondylen den Großteil ihrer Kontaktstrecke nach dorsal bereits in der Flexion bis etwa $25^{\circ}$ zurück (Pinskerova et al. 2004). Kinematisch heißt das, dass in diesem Bereich die Gelenkflächen überwiegend aufeinander abrollen.

In weiterer Flexion gleitet das Tibiaplateau schließlich lediglich um die Femurkondylen herum, so dass die beiden Gelenkkontakte auf dem Tibiaplateau ortsfest bleiben (Miller et al. 1998, Pandit et al. 2005). Die Roll-Gleitbewegung hindert die Femurkondylen bei der Kniebeugung am Überrollen der Meniskushinterhörner bzw. der Tibiahinterkanten (Strobel und Stedtfeld 1988).

Die Stabilität im TFG kommt bei Belastung durch die Kompression der Gelenkflächen zustande. Kompressiv wirkende Kräfte sind dabei die Schwerkraft und der Muskeltonus. Eine mechanisch stabile Gleichgewichtslage kann dabei jedoch nur durch inkongruente Gelenkflächen erreicht werden (KubeinMeesenburg et al. 1993).

Sagittale Schnitte durch das Tibiaplateau zeigen, dass das mediale Kompartiment eine konkave und das laterale Kompartiment eine konvexe Krümmung aufweist (Nägerl et al. 2008). Durch die Krümmungsmorphologie beider tibialer Gelenkflächen ist eine mechanisch stabile ExtensionsFlexionsbewegung in der sagittalen Hauptfunktionsebene vorgegeben und die Bewegung der Tibia nach dorsal beschränkt (Nägerl et al. 1993).

Die Menisken beeinflussen nicht unmittelbar die Stabilität und die Führung im belasteten Kniegelenk. Die Menisken sind beweglich und passen sich bei Belastung den komprimierten und damit stabilen inkongruenten Gelenkflächen an (Markolf et al. 1981, Nägerl et al. 1993).

Ebenfalls sind die Kreuzbänder nicht direkt an der Stabilität im belasteten Kniegelenk beteiligt. Die Kreuzbänder stabilisieren das Kniegelenk jedoch im unbelastenden Zustand sowie bei einwirkenden Scher- und Rotationskräften auch unter Gewichtsbelastung.

Im Ansatz- und Ursprungbereich des vorderen Kreuzbandes finden sich Ruffini-Körperchen, VaterPaccini-Körperchen und freie Nervenendigungen (Halata und Haus 1989, Zimny et al. 1986). 
Die Kreuzbänder dienen unter anderem auch als Sensorsystem, das über einen polysynaptischen Reflexbogen den Muskeltonus so einstellt, dass durch einen kompressiven Kraftschluss des Kniegelenks eine stabile Gelenkführung erreicht wird. Die propriozeptiven Eigenschaften der Kreuzbänder, lösen bei Dehnung eine Reflexantwort aus, die durch den entsprechenden Spannungszustand der Muskulatur eine Kompression von Tibia und Femur zur Folge haben. Über diesen Mechanismus wirken die Kreuzbänder kniegelenkstabilisierend (Nägerl et al. 1993).

Das Sensorsystem kann bei einwirkendem Stress jedoch auch selbst das VKB schützen, indem bei Dehnung des vorderen Kreuzbandes eine Reflexantwort ausgelöst wird, die zu einer Kontraktion der Mm. biceps femoris und semimembranosus führt (Schultz et al. 1984). Beide Muskeln gehören zur ischiokrualen Muskulatur und wirken agonistisch zum VKB, indem sie die Tibia nach dorsal ziehen. Dieser Reflexbogen, der schützend auf das VKB wirkt, wird als Ligament Cruciatum Anterius (LCA)Reflex bezeichnet (Gruber et al. 1986).

\subsection{Anatomie und Funktion des vorderen Kreuzbandes}

Das vordere Kreuzband entspringt im hinteren Viertel an der Innenfläche des Condylus lateralis femoris und verläuft nach antero-medial durch die Fossa intercondylaris. Es inseriert an der Mitte des Tibiaplateaus im Bereich der Eminentia intercondylaris.

Das vordere Kreuzband wird in ein anteromediales (AM) und in ein posterolaterales (PL) Faserbündel unterteilt. Beide Kreuzbänder sind, da entwicklungsgeschichtlich von dorsal in das Kniegelenk eingewandert, nur ventral von der Membrana synovialis bedeckt. Sie liegen daher intraartikulär aber retrosynovial (Girgis et al. 1975).

Kein Faserbündel des VKB verhält sich isometrisch und ist über den gesamten Bewegungsumfang angespannt. Je nach Stellung des Gelenks haben verschiedene Faserportionen des Bandes eine unterschiedlich starke Spannung (Amis und Dawkins 1991).

Das PL Bündel spannt sich erst bei endgradiger Extension an, das AM Bündel ist eher in Beugung gespannt (Furman et al. 1976).

Das VKB begrenzt die ventrale Translation der Tibia relativ zum Femur (Butler et al. 1980). Die APTranslation wird hauptsächlich vom anteromedialen Bündel beschränkt. Mit dem Lachman-Test kann die AP-Translation im unbelastenden Knie untersucht werden (Zantop et al. 2007).

Biomechanischen Untersuchungen haben gezeigt, dass das PL Bündel an der Beschränkung der Rotation des Kniegelenks mitbeteiligt ist (Gabriel et al. 2004). Das AM Bündel beteiligt sich dagegen kaum an der Rotationssicherung des Kniegelenks (Zantop et al. 2007).

Mit Hilfe des Pivot Shift Test kann eine Rotationsinstabilität des Kniegelenks bei ligamentärer Insuffizienz des VKB nachgewiesen werden. Dabei übt der Untersucher beim liegenden Patienten mit einer Hand einen Innenrotationsstress aus, mit der anderen Hand beugt der Untersucher das Knie und 
setzt es unter Valgusstress. Bei Insuffizienz des VKB, kommt es bei zunehmender Flexion meist ruckartig zur spürbaren Reposition des vorher nach ventral subluxierten lateralen Tibiaplateaus (Galway und MacIntosh 1980). Bei Innenrotation wickeln sich beide Kreuzbänder umeinander und limitieren somit eine übermäßige Einwärtsdrehung (Gruber et al. 1986).

Bei der Außenrotation wickeln sich die beiden Kreuzbänder auseinander. Die hemmende Wirkung der Kreuzbänder auf die Innenrotation ist demnach größer als für die Außenrotation. Bei Extension verläuft das VKB durch das Dach der Fossa intercondylaris und hemmt zusammen mit anderen Kapselbandstrukturen die Überstreckung des Kniegelenks, indem es bei Hyperextension am Dach der Fossa intercondylaris anschlägt (Strobel und Stedtfeld 1988).

\subsection{Problemstellung}

In der BRD ereignen sich pro Jahr ca. 35000 vordere Kreuzbandrupturen, von denen rund 28000 operativ versorgt werden. Über die Entwicklung der Inzidenz in der BRD gibt es leider keine aufschlussreichen Daten (Krudwig 2000), jedoch ist bekannt, dass in den USA der Ersatz des vorderen Kreuzbandes nach VKB-Ruptur zwischen 1991 und 1999 von 63000 Eingriffen pro Jahr auf über 100000 Eingriffe pro Jahr angestiegen ist und die Anzahl der Eingriffe weiterhin zunimmt (Brown und Carson 1999). Mit zunehmender operativer Therapie der vorderen Kreuzbandruptur nimmt daher die Bedeutung der Revisionschirurgie am vorderen Kreuzbandersatz zu.

Die vordere Kreuzbandruptur kann sowohl konservativ als auch operativ behandelt werden. Ein chirurgischer Therapieansatz ist dabei vor allem bei sportlich aktiven Patienten indiziert (Linko et al. 2005).

Sportlich ambitionierte Patienten mit vorderer Kreuzbandersatzplastik, die zu ihrem ursprünglichen sportlichen Aktivitätslevel zurückkehren, haben ein erhöhtes Risiko erneut eine Ruptur des Kreuzbandersatztransplantates zu bekommen. So ist bei 5-10\% der Patienten, die zu ihrem ursprünglichen sportlichen Aktivitätslevel zurückkehren, ein Revisionseingriff nach vorderer Kreuzbandrekonstruktion notwendig (Johnson et al. 1996, Wolf und Lemak 2002).

Von besonderer Bedeutung bei der Revisionschirurgie ist die Erweiterung der angelegten Bohrkanäle, auch Tunnelenlargement genannt, nach primär erfolgtem chirurgischem Eingriff. Mehrere Studien konnten zwar zeigen, dass das Tunnelenlargement keinen signifikanten Einfluss auf das primäre klinische Ergebnis hat (Clatworthy et al. 1999, L'Insalata et al. 1998), jedoch erschweren erweiterte Bohrkanäle den chirurgischen Revisionseingriff (George et al. 2006, Battaglia und Miller 2005). Die genaue Ursache des Tunnelenlargement ist unbekannt (Höher et al. 1998). Diskutiert werden mechanische und biologische Faktoren. Zu den mechanischen Ursachen gehören die frühfunktionelle Rehabilitation nach VKB-Ersatzplastik sowie ein bewegliches Transplantat im Bohrkanal. 
Majewski et al. konnten nachweisen, dass bei VKB-Rekonstruktion mit einem Transplantat aus der Patellarsehne mit zwei Knochenblöcken das tibiale Tunnelenlargement signifikant bei gelenkferner Fixation zunimmt. Die Autoren empfehlen daher eine gelenknahe an der anatomischen Insertion des vorderen Kreuzbandes gelegene Fixation (Majewski et al. 2002). Ist das Kreuzbandtransplantat nicht gelenknah fixiert, kann es zum vermehrten Tunnelenlargement aufgrund von Bewegungen des Transplantates in der Sagittalebene kommen. L'Insalata et al. nannten dies den „windshield wiper effect" (L'Insalata et al. 1998, S. 235). Ebenso wurde bei gelenkfernen Fixationstechniken eine Längenänderung des Transplantates durch dessen Eigenelastizität beobachtet. Dies wird als „bungee effect" bezeichnet (Höher et al. 1998, S. 237). Der „bungee effect" kann zum verzögerten Einheilen des VKB-Ersatztransplantates und zum Tunnelenlargement führen (Uchio et al. 2002).

Buelow et al. zeigten in einer Vergleichsstudie, dass eine gelenknahe Fixation mit Interferenzschrauben zum vermehrtem Tunnelenlargement initial, das heißt direkt postoperativ, im Vergleich zu gelenkfernen Fixationstechniken (Endobutton) führt, da das Eindrehen der Schraube selbst den Bohrkanal aufweitet. Jedoch wird im weiteren postoperativen Verlauf ein Tunnelenlargement bei gelenknahen Fixationstechniken (Interferenzschrauben) im Vergleich zu gelenkferner Fixation (Endobutton) reduziert (Buelow et al. 2002). Durch die Anwendung von Interferenzschrauben ist eine gelenknahe Fixation des Transplantates möglich. Dies verhindert frühe Mikrobewegungen des Transplantates im Bohrkanal, führt zu einem stabilem Kontakt zwischen Transplantat und Bohrkanalwand, dichtet den Bohrkanal gegen das Eindringen von Synovialflüssigkeit ab und vermindert im weiteren postoperativen Verlauf das Tunnelenlargement (Buelow et al. 2002, Höher et al. 1998). Seit Mitte der goer Jahre ist die frühfunktionelle Rehabilitation Standard in der Nachbehandlung der VKB-Rekonstruktion (Shelbourne und Nitz 1990). Das Protokoll beinhaltet Krafttraining, frühe Rückkehr zur sportlichen Aktivität und frühes Erreichen des physiologischen Bewegungsumfanges des Kniegelenks. Neben den vielen Vorteilen der frühfunktionellen Rehabilitation konnte darunter jedoch eine Zunahme des Tunnelenlargements beobachtet werden. Demnach verzögert die frühfunktionelle Rehabilitation möglicherweise ein Einheilen des Transplantates und kann zum Tunnelenlargement führen (Hantes et al. 2004).

Eine weitere Ursache für das Tunnelenlargement kann ein erhöhter Zytokin-Spiegel in der Synovialflüssigkeit des Kniegelenks sein. Zytokine erhöhen sich infolge einer VKB-Ruptur (Cameron et al. 1997, Cameron et al. 1994), können mit der Synovialflüssigkeit in die Bohrkanäle eindringen und dort zu Osteolysen führen (Schamalzried et al. 1997, Friedman et al. 1994).

Zur Befestigung des Kreuzbandersatztransplantates im femoralen und tibialen Bohrkanal nach VKBRuptur können bioabsorbierbare oder metallische Schrauben verwendet werden. In unterschiedlichen Studien konnte gezeigt werden, dass es keine Unterschiede im klinischen Ergebnis bei Verwendung beider Schraubenarten gibt (Barber et al. 1995, Warden et al. 1999). 
Da Interferenzschrauben aus Metall aufgrund ihrer scharfen Kanten zu Transplantatirritationen (Zantop et al. 2006) führen und das Entfernen von Interferenzschrauben aus Metall bei einem möglichen Revisionseingriff problematisch sein kann (Kurzweil et al. 1995), werden vermehrt bioabsorbierbare Fixationsmaterialien in der Kreuzbandchirurgie verwendet.

Es stehen mittlerweile eine Vielzahl verschiedener bioresorbierbarer Schrauben mit unterschiedlicher Materialzusammensetzung zur Verfügung. So sind zum Beispiel Schrauben aus polyglycolic acid (PGA), poly-L lactic acid (PLLA) oder poly-D lactic acid (PDLLA) erhältlich.

Die mittlere Volumenabnahme von poly- $L$ lactid acid (PLLA) Schrauben beträgt nach zwei Jahren $64 \%$ für die femoralen und 63\% für die tibialen Schrauben (Drogset et al. 2006).

Osteolysen werden in 16\% (Drogset et al. 2006), sterile Zysten in 5-10\% der Fälle beschrieben (Macarini et al. 2004). Fremdkörperreaktionen werden nach Implantation von bioresorbierbaren Schrauben ebenfalls beschrieben (Böstman et al. 1990).

Ein Ersatz der Schrauben durch Knochengewebe wurde bisher beim Menschen nur vereinzelt im Langzeitverlauf beobachtet (Lajtai et al. 2001). Demgegenüber zeigen mehrere Studien, dass auch langfristig kein Ersatz der bioresorbierbaren Schrauben durch Knochengewebe nachgewiesen werden konnte (Tecklenburg et al. 2006, Warden et al. 1999, Bach et al. 2002, Barber und Dockery 2006). Aus diesem Grunde werden mittlerweile bioabsorbierbare Materialien verwendet, denen osteokonduktive Substanzen hinzugefügt sind.

Nach Angaben des Herstellers besitzt die bioabsorbierbare Milagro ${ }^{\circledR}$-Interferenzschraube für die Transplantatfixation beim vorderen Kreuzbandersatz ein optimiertes Abbau- und Integrationsverhalten in den Knochen. Die Milagro ${ }^{\circledR}$-Schraube besteht zu 30\% aus ß-TCP (TriCalcium Phosphat) und zu 70\% aus Poly(lactid-Co-) Glycolid. Es ist bekannt das ß-TCP osteokonduktiv wirkt und vollständig durch Knochengewebe ersetzt wird (Bodde et al. 2007). Durch die Mischung von resorbierbaren Polymeren mit ß-TCP konnten in experimentellen Studien die biomechanischen Eigenschaften, das Resorptionsverhalten und die Osteokonduktivität beider Materialien verbessert werden (Montjovent et al. 2007). Dieser Effekt sollte auch für die Milagro ${ }^{\circledR}$-Interferenzschrauben genutzt werden. In vorklinischen Tierstudien konnte nachgewiesen werden, dass die Milagro ${ }^{\circledR}$-Interferenzschraube vollständig bioabsorbierbar ist (http://www.jnjgateway.com/home.jhtml?loc=USENG ). Vergleichbare Studien, die sowohl die klinischen Ergebnisse als auch die Biokompatibilität der Milagro ${ }^{\circledR}$ Interferenzschraube beim Menschen beinhalten, gibt es derzeit nicht.

Die Sehnentransplantatwahl ist eine zentrale Frage in der Kreuzbandchirurgie. Etabliert haben sich die Verwendung des mittleren Drittels der Patellarsehne sowie der Semitendinosussehne (ST) alleine oder in Kombination mit der Gracilissehne (STG). In vorliegender Studie wurde die VKB-Rekonstruktion in arthroskopischer Technik mit der ST oder der STG als Transplantat durchgeführt. Vergleichsstudien, die 
das klinische Ergebnis des VKB-Ersatzes mit alleiniger Semitendinosussehne versus Semitendinosussehne in Kombination mit der Gracilissehne vergleichen, sind rar.

Einzig Gobbi et al. verglichen das klinische Ergebnis der VKB-Rekonstruktion mit der ST versus STG als Transplantat. Die Autoren konnten zeigen, dass es keine signifikanten Unterschiede im klinischen Outcome gibt und empfehlen daher auf die Gracilissehne als Transplantat, falls möglich, zu verzichten (Gobbi et al.2005). Im Unterschied zur vorliegenden Arbeit erfolgte die Transplantatfixation hierbei femoral mit einem EndoButton. Unklar ist, ob die Fixation mit bioresorbierbaren Interferenzschrauben das klinische Ergebnis von ST versus STG als Transplantat beeinflusst. In vorliegender Studie wurde daher eine Patientengruppe, deren Kreuzbandersatztransplantat alleine aus der ST gefertigt wurde, einer Patientengruppe, deren Kreuzbandersatztransplantat aus der STG besteht, gegenübergestellt. Untersucht wurden Patienten, die zwischen 06/2005 bis 10/2006 eine mit Milagro ${ }^{\circledR}$ Interferenzschrauben befestigte vordere Kreuzbandplastik aus ST oder STG erhalten haben.

\section{$1.4 \quad$ Fragestellung}

Im Rahmen dieser Arbeit sollte folgenden Fragestellungen nachgegangen werden:

1.) Wie sind generell die klinischen Ergebnisse bei Transplantatfixation mittels femoraler und tibialer Milagro ${ }^{\circledR}$-Interferenzschraube?

2.) Gibt es Unterschiede im klinischen Ergebnis bei VKB-Rekonstruktion mit der Semitendinosussehne alleine versus Semitendinosus- und Gracilissehne als Transplantat?

3.) Kann bei Transplantatfixation mit bioresorbierbaren Schraubensystemen auf die Gracilissehne verzichtet werden?

4.) Gibt es bei Messung mit dem KT 1000 Arthrometer signifikante Unterschiede in der ventralen Translation der Tibia bei Verwendung der ST versus STG als Kreuzbandtransplantat?

Bei den MRT-Untersuchungen sollte folgenden Fragestellungen nachgegangen werden:

1.) Kommt es zur Biodegradation der Milagro ${ }^{\circledR}$-Interferenzschraube im menschlichen Organismus?

2.) Wie ist der zeitliche Ablauf der Biodegradation der Milagro ${ }^{\circledR}$-Interferenzschrauben?

3.) Kommt es im MRT zu Zeichen der Fremdkörperreaktion, infolge der Biodegradation?

4.) Kommt es zum femoralen und tibialen Tunnelenlargement bei Fixation mit Milagro ${ }^{\circledR}$ Interferenzschrauben?

5.) Wird die sich abbauende Schraube durch Knochengewebe ersetzt und

6.) kann dadurch das Tunnelenlargement reduziert werden? 


\section{Material und Methoden}

\subsection{Patientenkollektiv und Ausschlusskriterien}

Im Zeitraum von Juni 2005 bis Oktober 2006 wurden 37 Patienten mit vorderer Kreuzbandplastik in die Studie aufgenommen. Die Patienten wurden prospektiv nicht randomisiert und erhielten entweder eine Kreuzbandersatzplastik mittels Semitendinosussehne oder mittels Semitendinosus- und Gracilissehne. Die Rekonstruktion des vorderen Kreuzbandes erfolgte bei 16 Patienten mit alleiniger Semitendinosussehne, bei 21 Patienten wurde die Kreuzbandersatzplastik mit der Semitendinosus- und zusätzlicher Gracilissehne durchgeführt.

Ein Patientenalter von über 50 Jahren, gravierende medizinische Grundleiden, Knorpelläsionen (ICRS Grad II.-IV.) mit einem Ausmaß größer als $3 \mathrm{~cm}^{2}$, Kniegelenksluxationen, Patellaluxationen, zusätzliche hintere Kreuzbandruptur, autologe Chondrozytentransplantation, Collagenmeniskusersatz sowie Meniskusteilresektionen größer 2/3 stellten Ausschlusskriterien für die Studie dar.

\subsection{Verletzungsursachen, Zeit zwischen Unfall und Operation}

Die Verletzungsursachen der vorderen Kreuzbandruptur wurden anhand der „Innsbrucker Knie Sportfähigkeitsklassifikation" (Fink et al. 1993) in drei Sportartengruppen unterteilt. Dieses Klassifikationsschema ordnet alle Sportarten hinsichtlich der Gefährdung der Kniestabilität in folgende Sportarten ein:

- High-Risk-Pivoting-Sportarten ${ }^{1}$

- Low-Risk-Pivoting-Sportarten ${ }^{2}$

- Non-Risk-Pivoting-Sportarten ${ }^{3}$.

\footnotetext{
${ }^{1}$ High-Risk-Pivoting-Sportarten: Sportarten, die durch einen hohen Anteil plötzlicher Stopps, schneller Drehungen, unkontrollierten Sprungbewegungen und hoher mechanischer Belastungen für das Kniegelenk charakterisiert sind, z.B. Fussball.
}

${ }^{2}$ Low-Risk-Pivoting-Sportarten: Sportarten, in denen Drehbewegungen vorkommen können, aber nicht von vornherein charakteristisch für diese Sportart sind, z.B. Joggen.

${ }^{3}$ Non-Risk-Pivoting-Sportarten: Sportarten, die durch kontrollierte Bewegungen unter nur teilweiser Belastung des Körpergewichts gekennzeichnet sind, z.B. Schwimmen.

Unter Sonstiges wurden vordere Kreuzbandverletzungen zusammengefasst, die sich während Beruf oder Freizeit ereigneten.

Der Zeitraum vom Unfall bis zur Operation des vorderen Kreuzbandes wurde eingeteilt in akut ( $<2$ Wochen), subakut (2-8 Wochen) und chronisch (> 8 Wochen) (Hefti und Müller 1993). 


\subsection{Milagro ${ }^{\circledR}$-Interferenzschraube}

Die Milagro ${ }^{\circledR}$-Interferenzschraube besteht zu 30\% aus ß-TCP (TriCalcium Phosphat) und zu 70\% aus Poly(lactid-Co-) Glycolid. Sie ist in Größen von 7-10 mm Durchmesser und einer Länge von jeweils 23 oder $30 \mathrm{~mm}$ erhältlich und dient zur Fixation der Kreuzbandplastik im tibialen und femoralen Bohrkanal. Die Schraube ist kanüliert und kann somit exakt über einen Führungsdraht eingebracht werden. Das Eindrehen der Schraube erfolgt mit einem dafür speziell gefertigten Schraubendreher.

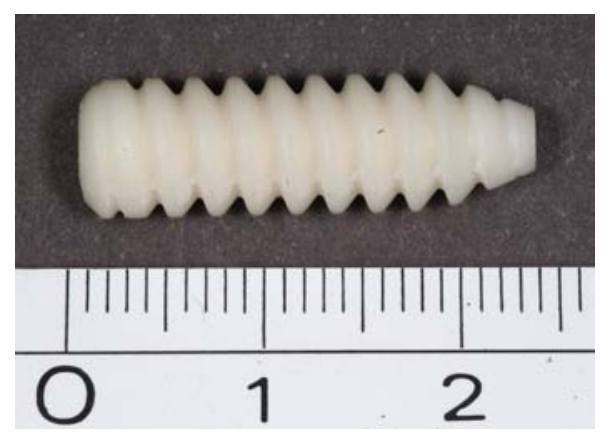

Abbildung 1 aus Frosch et al. (2009, S.74): Milagro ${ }^{\circledR}$-Interferenzschraube (7 x $23 \mathrm{~mm}$ )(Mit freundlicher Genehmigung des Springer Verlages).

\section{$2.4 \quad$ Operationsverfahren}

Der Ersatz des vorderen Kreuzbands wurde von zwei erfahrenen Fachärzten in arthroskopischer Technik durchgeführt. Die Operation erfolgte in Vollnarkose. In Rückenlage wurde ein elektrischer Beinhalter verwendet. Intraoperativ wurde an dem verletzten Bein eine Oberschenkelblutsperre mit einem maximalen Druck von $350 \mathrm{mmHg}$ angelegt.

\subsubsection{Diagnostische Arthroskopie}

Zunächst wird über einen anterolateralen Zugang das Arthroskop in das Kniegelenk eingeführt. Nach Anlage eines anteromedialen Arbeitszuganges erfolgt die diagnostische Arthroskopie, um das Kniegelenk hinsichtlich weiterer Kniebinnenschäden (Meniskusschaden, Knorpelschaden) zu untersuchen. Mit Hilfe eines Shavers werden die Reste des vorderen Kreuzbandes femoral vollständig entfernt. Tibial bleibt ein bis zu 1,5 cm langer Kreuzbandstumpf stehen, um den tibialen Bohrkanal abzudichten und die Propriozeption soweit möglich zu erhalten.

\subsubsection{Transplantatentnahme}

Über einen ca. $3 \mathrm{~cm}$ langen in Längsachse der Sehnen verlaufenden Schnitt über dem Pes anserinus werden die Hamstringsehnen dargestellt. Im amerikanischen Sprachraum werden die Sehnen des Pes anserinus als „Hamstrings" bezeichnet. 
Anschließend folgt nach Darstellen der Sehne die Entnahme derselben als freies Transplantat mit Hilfe eines Sehnenstrippers (Abbildung 2).

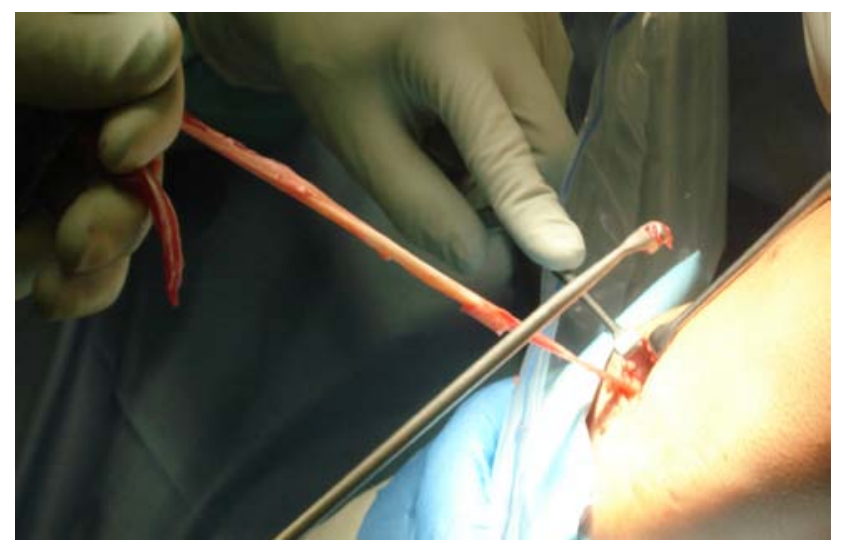

Abbildung 2: Entnahme einer Hamstringsehne

\subsubsection{Sehnenpräparation}

Ein Assistent bereitet die Hamstringsehne zur Transplantation vor. Zunächst wird sie von anhängendem Muskel- und Synovialgewebe befreit (Abbildung 3 a). Anschließend erfolgt die Anordnung des Transplantates zu einer Dreifach- oder Vierfachschlinge. Mit Hilfe von Zugfäden an den Schlaufenenden wird das Transplantat mit 150 Newton auf einem Präparationsbrett vorgespannt (Abbildung 3 b). Anschließend werden die Sehnen jeweils femoral und tibial mit Orthocord ${ }^{\circledR}$ Fäden vernäht.
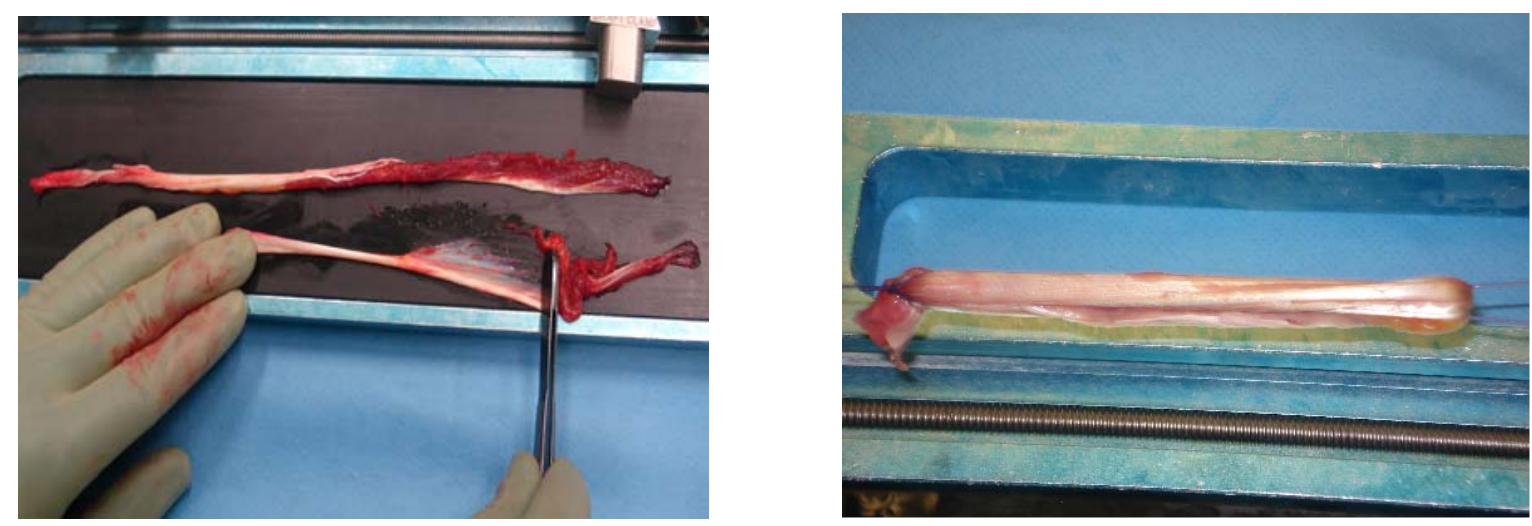

Abbildung 3 a/b: Präparation der Semitendinosus- und Gracilissehne (a) und Vorspannen der Vierfachsehnenschlinge mit 150 Newton (b)

\subsubsection{Anlage der Bohrkanäle}

Der tibiale Bohrkanal wird in $90^{\circ}$ Kniebeugung angelegt. Zunächst wird mit einem tibialen Zielgerät, dessen Spitze vor dem Ansatz des hinteren Kreuzbandes liegt, ein Ösen - Draht vom medialen Tibiakopf in $55^{\circ}$ ansteigenden Winkel in das Kniegelenk eingebracht. Anschließend wird der 
Führungsdraht mit einem kanülierten Bohrer überbohrt (Abbildung 4 a). Der Durchmesser des tibialen und femoralen Bohrkanals wird dabei bis auf o,5 mm exakt der Transplantatstärke angepasst.

Der femorale Kanal wird über den anteromedialen Gelenkzugang mittels Zielgerät in der 10:30 Uhr Position für das rechte oder in der 1:30 Uhr Position für das linke Knie angelegt (Abbildung 4 b).

Der femorale Kanal wird über das anteromediale Portal bei $120^{\circ}$ Kniebeugung bis ca. $2,5 \mathrm{~cm}$ tief eingebohrt

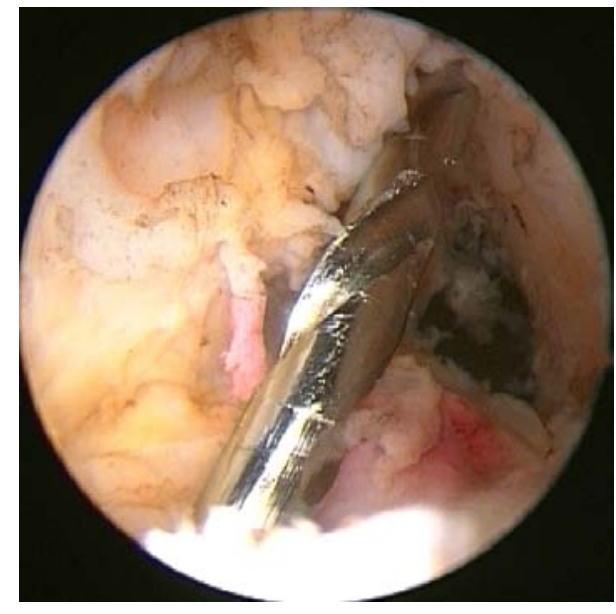

Abbildung 4a: Anlage tibialer Bohrkanal

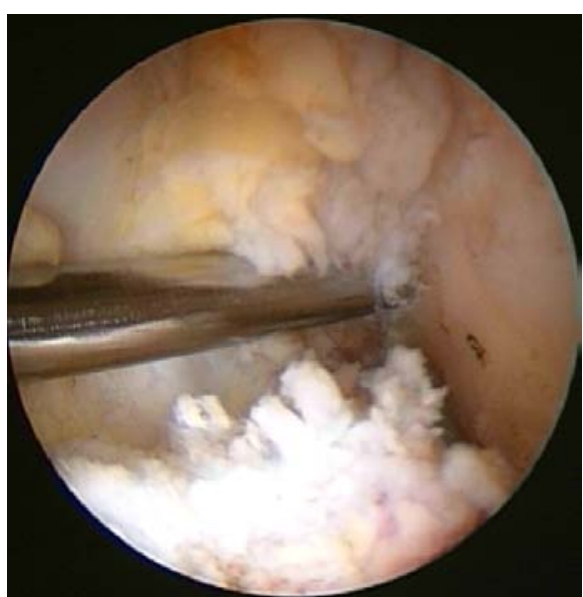

Abbildung 4b: Anlage femoraler Bohrkanal

\subsubsection{Transplantatplatzierung}

Das Kreuzbandersatztransplantat, das mit Fäden durch einen Ösendraht geschlungen wird, wird über den tibialen Bohrkanal eingezogen. Nach femoraler Schraubenfixation wir das Transplantat vorgespannt und das Kniegelenk mehrfach bewegt, bevor die tibiale Schraube möglichst gelenknah platziert wird. Abbildung 5 zeigt die Befestigung des Transplantates im tibialen Bohrkanal mit einer Milagro ${ }^{\circledR}$-Interferenzschraube. Der Durchmesser der Interferenzschrauben wurde gleich dem Bohrkanaldurchmesser oder 0,5 mm dünner gewählt.

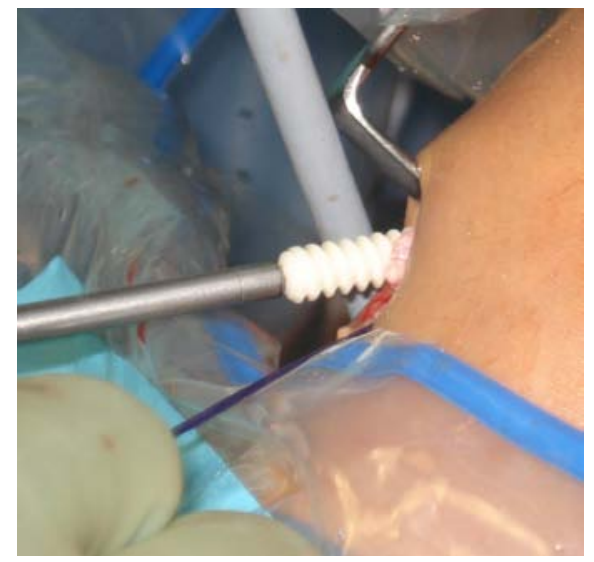

Abbildung 5: Tibiale Transplantatfixation 
Nach Befestigung des Transplantates mit den Interferenzschrauben wird das Kniegelenk mehrmals durchbewegt und arthroskopisch ein Impingement des Transplantates im Gelenk, speziell in der Notch überprüft. In Abbildung 6 ist die korrekte Lage der Kreuzbandersatzplastik zu sehen. Unter arthroskopischer Kontrolle wird abschließend die Stabilität der Kreuzbandersatzplastik mit dem Lachman-Test überprüft.

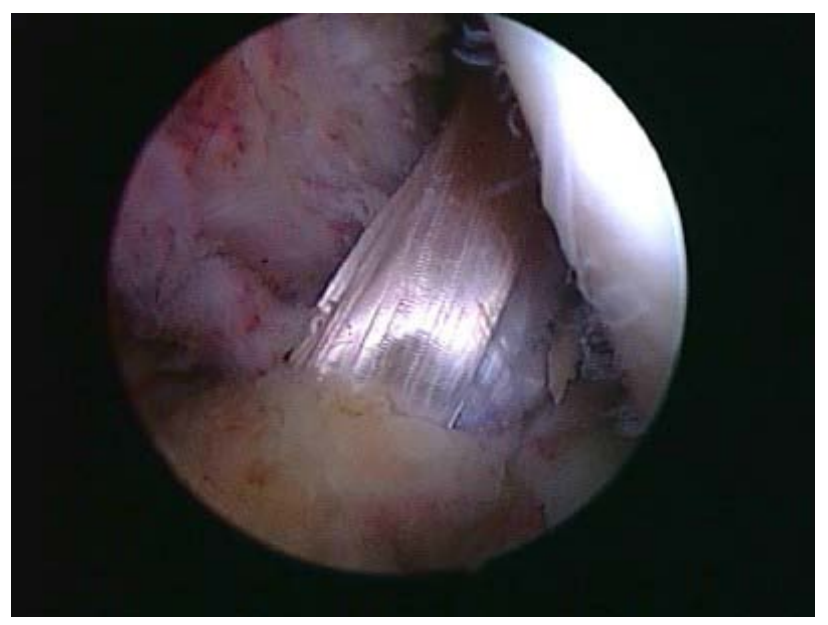

Abbildung 6: VKB-Ersatzplastik bei $90^{\circ}$ Beugung

\subsection{Nachbehandlung}

Alle Patienten wurden postoperativ mit Motorschiene, Krankengymnastik und Kälteanwendungen behandelt. Therapieziel der Frühphase (bis nach der 3.Woche) war die sofortige volle Extension. Die Beugung des Kniegelenks war bis $90^{\circ}$ erlaubt. Seitens der Physiotherapie kamen Patellamobilisation, Gelenkmobilisation, PNF, Lymphdrainage und Kryotherapie zur Anwendung (Frosch et al. 2001). Alle Übungen wurden nur in geschlossener Bewegungskette durchgeführt, die Patienten belasteten mit 10$20 \mathrm{~kg}$ teil. Ab der 5. postoperativen Woche standen die zunehmende Vollbelastung, unidirektionales Koordinationstraining, Standstabilisation, Bewegungsbad, medizinische Trainingstherapie und Muskelaufbautraining in geschlossener Kette im Vordergrund. Die Beweglichkeit wurde zunehmend freigegeben. Ab der 7. Woche erfolgte multidirektionales Koordinationstraining auf labiler Unterlage (z. B. Minitrampolin, Weichbodenmatte) und vorsichtiges Joggen auf ebenem Boden.

Krankengymnastik mit offener Bewegungskette wurde erst ab der 12. Woche erlaubt. Bei zusätzlichen Verletzungen (Meniskus, Seitenbänder) variierte das Nachbehandlungsregime entsprechend der Verletzung. Bei begleitender Seitenbandinstabilität kam eine Knieorthese für 6 Wochen zur Anwendung. 


\subsection{Kniegelenkfunktionsscores}

\subsubsection{Tegner Aktivitätsskala}

Der Tegner Score wurde verwendet, um das Aktivitätslevel des Patienten zu bestimmen. Die Patienten gaben ihr präoperatives Aktivitätsniveau und das Aktivitätsniveau zwölf Monate nach Ersatz des vorderen Kreuzbandes an.

Die Aktivitäten werden vom Patienten in elf Stufen auf einer Skala von "0" bis "10" eingeteilt. Die Skala reicht in aufsteigender Reihenfolge von Aktivitäten des täglichen Lebens bis hin zum Wettkampfsport auf nationalem und internationalem Niveau mit und ohne Gegnerkontakt (Tegner und Lysholm 1985).

\subsubsection{Lysholm Score}

Lysholm und Gillquist stellten 1982 ein Auswertungsschema vor, dessen Gesamtergebnis hauptsächlich durch die subjektive Beurteilung des Patienten zustande kommt. Die maximal erreichbare Punktzahl beträgt 100 .

Dem Patienten wurden Fragen zu den folgenden Problembereichen gestellt, die unterschiedlich mit Punkten gewichtet werden:

1.Hinken 2. Einsatz von Gehhilfen $\quad$ 3.Treppensteigen $\quad$ 4.tiefe Hocke $\quad$ 5.Instabilität 6.Schmerz 7. Schwellung

Die Interpretation der Gesamtpunktzahl wurde wie folgt festgelegt: 95 bis 100 - exzellent, 84 bis 94 - gut, 65 bis 83 - zufriedenstellend, weniger als 65 - schlecht (Lysholm und Gillquist 1982).

\subsubsection{Marshall Score}

Der Marshall-Score setzt sich aus anamnestischen Angaben, funktionellen Aspekten, dem Oberschenkelumfang und der klinischen Untersuchung, die 30 von 50 Punkten einnimmt, zusammen. 20 der 30 Punkte der klinischen Untersuchung kommen der Bandstabilität zu. Jeweils fünf Punkte werden bei stabilem lateralen und medialen Kollateralband sowie stabilem hinterem und vorderem Kreuzband vergeben.

Weitere drei Punkte können durch ein normales Bewegungsausmaß, ebenfalls 3 Punkte bei normaler Muskelkraft erlangt werden. Insgesamt vier Punkte können vergeben werden, wenn kein Druckschmerz, kein Gelenkerguss, keine Weichteilschwellung und keine Krepitation vorliegen (Marshall et al 1977).

Durch die Verteilung der Punkte steht bei diesem Bewertungsscore die Stabilität der Kniestabilisatoren im Vordergrund, die subjektive Einschätzung des Patienten wirkt sich auf das Gesamtergebnis eher gering aus. 
Die Interpretation der Gesamtpunkzahl wurde wie folgt festgelegt:

41 bis 50 Punkte - sehr gut, 36 bis 40 Punkte - gut, 31- 35 Punkte - mittelmäßig, <30 Punkte mangelhaft.

\subsubsection{Visuelle Analogskala Schmerz und Funktion}

Alle Patienten gaben ihre Schmerzintensität auf einer visuellen numerischen Analogskala von 0-10 an. O entsprechen keine Schmerzen bei dem Patienten, 10 entsprechen maximal starke Schmerzen des Patienten.

Ebenfalls gaben alle Patienten ihre subjektiv empfundene Funktionalität des Kniegelenks an. Gibt der Patient eine 10 an, entspricht dies einer nicht eingeschränkten Funktionalität. Gibt der eine o an, entspricht dies einer maximal eingeschränkten Funktionalität vergleichbar mit einem versteiften Kniegelenk.

\subsubsection{IKDC-Evaluationsblatt}

Das „International Knee Documentation Comittee” (IKDC) entwickelte das IKDC-Evaluationsblatt (Hefti und Müller 1993). Das IKCD Blatt ist in acht Ergebnisbereiche untergliedert, wobei lediglich folgende vier Bereiche in das Gesamtergebnis eingehen:

a) Subjektive Beurteilung

b) Fehlende Symptome bei Aktivitätsniveau

c) Bewegungsumfang

d) Bandapparat.

Die Problembereiche a) und b) werden vom Patienten, die Problembereiche c) und d) vom Untersucher bewertet. Die einzelnen Bereiche werden nicht numerisch bewertet. Jeder Bereich wird mit (A) normal, (B) fast normal, (C) abnormal und (D) stark abnormal qualifiziert. Die schlechteste Bewertung aus a)-d) bestimmt das Gesamtergebnis. Ein Patient, der nicht in allen Parametern einer Gruppe ein „normal” (A) erreicht, kann auch im Gesamtergebnis kein „normal” (A) erreichen.

Vier weitere Bereiche werden dokumentiert: kompartimentelle Befunde, Symptome an der Transplantatentnahmestelle, funktionelle Testung und radiologische Befunde (im Rahmen der Nachuntersuchung nicht erfolgt). 


\subsection{KT 1000 Arthrometer}

Das KT 1000 Kniearthrometer (Firma Medmetric / Kalifornien/ USA) ermöglicht eine instrumentelle Messung der ventralen Translation der Tibia relativ zum Femur (Daniel et al. 1985).

Abbildung 7 veranschaulicht die Durchführung der Untersuchung. Die Messungen wurden bei einem Kniebeugewinkel von ca. $20^{\circ}$ durchgeführt, da hierbei die Patella bestmöglich in der femoralen Trochlea stabilisiert ist. Fehlabmessungen, bedingt durch eine erhöhte Mobilität der Patella können somit weitgehend vermieden werden.

Bei der Messung wird die Patella mit einer Hand stabilisiert, während mit der anderen Hand an dem Handgriff gezogen wird, bis ein erster Piepton zu hören ist. Dieser gibt eine Zugkraft von 67 Newton an. Der zweite Piepton ertönt bei 89 Newton. Die maximale passive vordere Schublade wurde durch direkten Zug am Unterschenkel von dorsal bestimmt. Die maximale passive vordere Schublade wird allgemein als MMD (maximal manual drawer) bezeichnet.

Der tibiale Vorschub kann in Millimeter bei einer Zugkraft von 67 Newton, 89 Newton und beim MMD am KT 1000 Arthrometer abgelesen werden.

Jede dieser Messungen wurde auf einer Seite dreimal durchgeführt, daraus das arithmetische Mittel berechnet und mit der Gegenseite verglichen.

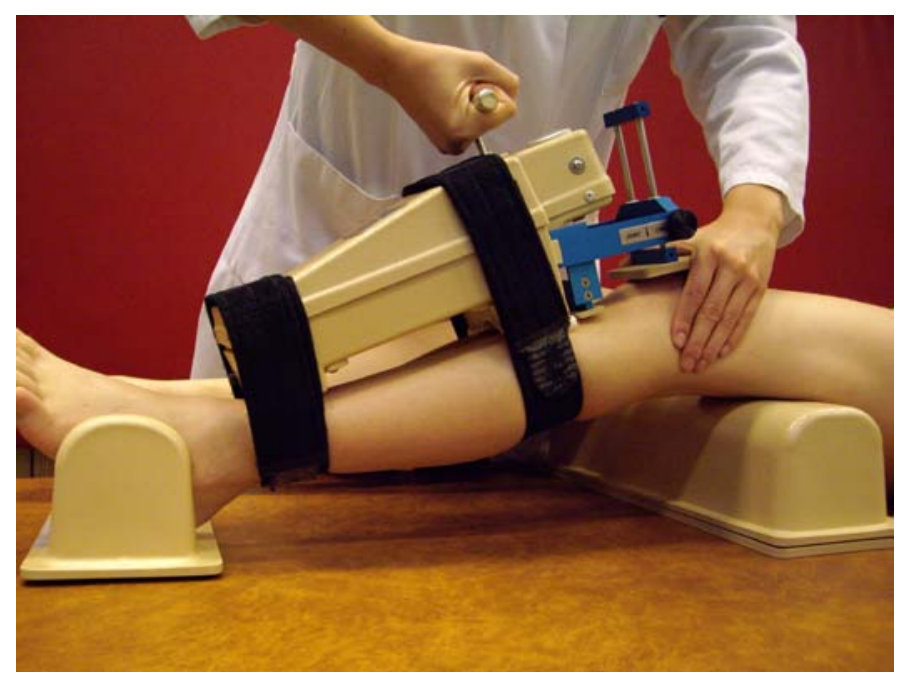

Abbildung 7: KT 1000 Arthrometer 


\subsection{Kernspintomographie}

\subsubsection{Methodik und Durchführung der Messung}

Aus dem Gesamtkollektiv wurden nach dem Zufallsprinzip zwölf Patienten ausgewählt, sieben mit einer Kreuzbandplastik aus Semitendinosus- und Gracilissehne, fünf Patienten mit alleiniger Semitendinosussehne als Transplantat. Nach drei, sechs und zwölf Monaten wurde eine MRTUntersuchung des operierten Kniegelenks durchgeführt.

Als Kernspintomograph wurde ein 1,5 Tesla MRT-Scanner (Magnetom Symphony and Sonata, Siemens Erlangen, Germany) verwendet. Am betroffenen Kniegelenk wurden T1 gewichtete spin echo (SE) Sequenzen in sagittaler Ebene, T2 gewichtete spin echo (SE) Sequenzen in sagittaler Ebene, Protonen gewichtete turbo spin echo Sequenzen (TSE) mit Fettsättigung und eine 3D-DESS Sequenz (double echo steady state) durchgeführt. Die Matrix der Bilder war 512 × 256, das Sichtfeld war in der 3D-DESS Sequenz und der Protonen gewichteten turbo spin echo Sequenz $180 \mathrm{~mm}$, in der T1 und T2 gewichteten Sequenz $220 \mathrm{~mm}$. Die Dicke der Bilder war entweder 1,5 mm oder $3 \mathrm{~mm}$. Es wurde gezielt nach dem Volumenverlust der Schrauben, Tunnelenlargement, Osteolysen, Flüssigkeitssäumen und Ödemen gesucht und evaluiert, ob im Verlauf die Schrauben durch Knochengewebe ersetzt werden.

Die Abbaurate der Interferenzschrauben wurde anhand eines standardisierten Protokolls in der 3DDESS Sequenz bestimmt. Das präoperative Schraubenvolumen ( $\left.\mathrm{V}_{1}\right)$ wurde näherungsweise mittels der Formel: $V_{1}=\pi r^{2} \times I$ kalkuliert, wobei $r$ der Radius und I die Länge der Schraube ist. Das postoperative Schraubenvolumen $\left(V_{2}\right)$ wurde näherungsweise mit der Formel $V_{2}=\pi\left(1 / 2\left(d_{1} / 2+d_{2} / 2\right)\right)^{2} \times \mid$ bestimmt, wobei $d_{1}$ der minimale Schraubendurchmesser, $d_{2}$ der maximale Schraubendurchmesser und I die maximal messbare Länge der Schraube im MRT ist. Der Volumenverlust der Schraube $\Delta V$ war: $\Delta \mathrm{V}=\mathrm{V}_{1}-\mathrm{V}_{2}$. Der Volumenverlust der Schraube in Prozent war: $\%=100 \% \times \Delta \mathrm{V} / \mathrm{V}_{1}$ (Drogset et al. 2006).

Das Tunnelenlargement wurde in der Sagittalebene der 3-D DESS Sequenz senkrecht zur Längsachse des Bohrkanals gemessen. Um den Durchmesser des ursprünglichen Bohrkanals zu bestimmen, wurde der Durchmesser des verwendeten Bohrers (Bohrstärke) verwendet. Zur Berechnung des Tunnelenlargements wurde der tibiale und femorale Bohrkanal in jeweils drei gleich lange Bohrkanalabschnitte unterteilt. Die Bezeichnung der Bohrkanalabschnitte lautete dabei: Gelenknaher, mittlerer und gelenkferner Bohrkanalabschnitt.

Der maximale Durchmesser eines Bohrkanalabschnitts wurde zur Berechnung des Tunnelenlargement eines Bohrkanalabschnittes verwendet. Das Tunnelenlargement eines Bohrkanalabschnitts wurde anhand folgender Formel bestimmt: $\%=100 \%$ x max. Durchmesser des Bohrkanals in einem Bohrkanalabschnitt in cm / Bohrstärke in cm. 
Die Berechung des arithmetischen Mittels aus dem gelenknahen, mittleren und gelenkfernen Tunnelenlargement ergab dann das durchschnittliche tibiale sowie femorale Tunnelenlargement. Die Berechnung des durchschnittlichen tibialen Tunnelenlargements erfolgte anhand folgender Formel: Durchschnittliches tibiales Tunnelenlargement $=1 / 3$ (Tunnelenlargement gelenknaher Bohrkanalabschnitt + Tunnelenlargement mittlerer Bohrkanalabschnitt + Tunnelenlargement gelenkferner Bohrkanalabschnitt).

Da das Tunnelenlargement der gelenknahen Bohrkanalabschnitte von Bedeutung für die Revisionschirurgie ist, wurden diese Bereiche gesondert ausgewertet.

\subsubsection{Evaluation der Messgenauigkeit}

Bei acht Patienten wurden bis zum dritten postoperativen Tag kernspintomographische Aufnahmen zur Bestimmung der Messgenauigkeit in der 3D-DESS Sequenz durchgeführt.

In der 3D-DESS Sequenz wurde die Länge und der Durchmesser der tibialen Interferenzschraube von einem Untersucher gemessen. Mit Hilfe der direkt postoperativ gemessenen Schraubenmaße konnte berechnet werden, inwieweit die gemessenen Werte in der 3D-DESS Sequenz von den tatsächlichen Werten abweichen. Die prozentuale Abweichung der Messung von den tatsächlichen Schraubenmaßen konnte dadurch berechnet werden. Die direkt postperativ durchgeführten Messungen gaben Hinweise über die Messgenauigkeit in der 3D-DESS Sequenz.

In Abbildung 8 ist eine tibiale Interferenzschraube in der 3D-DESS Sequenz drei Tage nach vorderer Kreuzbandplastik dargestellt.

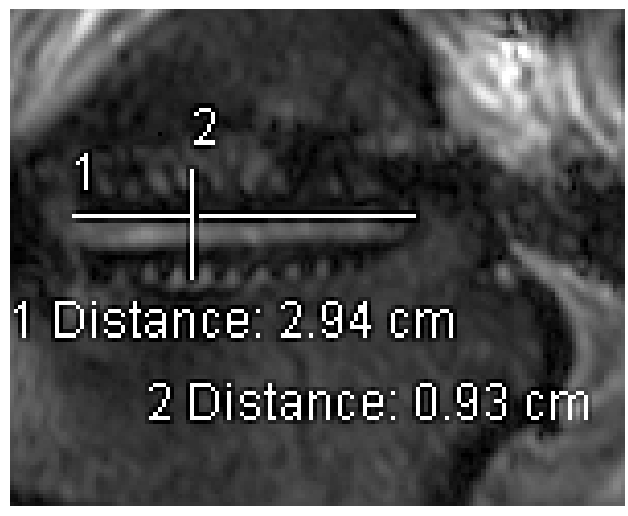

Abbildung 8: Milagro ${ }^{\circledR}$-Interferenzschraube in der 3D-DESS Sequenz

Die tatsächliche Schraubenlänge aus Abbildung 8 ist $\mathrm{I}_{\mathrm{t}}=3,00 \mathrm{~cm}$ und der tatsächliche Schraubendurchmesser $d_{t}=0,90 \mathrm{~cm}$. Die digital gemessenen Werte in der 3D-DESS Sequenz sind für die Länge der Schraube $I_{g}=2,94 \mathrm{~cm}$ und für den Durchmesser der Schraube $d_{g}=0,93 \mathrm{~cm}$. Die prozentuale Abweichung für die Schraubenlänge wurde nach folgender Formel berechnet: 
Prozentuale Abweichung der Schraubenlänge in \%: \% = $100 \% \times \mathrm{I}_{\mathrm{g}} / \mathrm{l}_{\mathrm{t}}$.

Entsprechend wurde die prozentuale Abweichung für den Schraubendurchmesser berechnet.

Für die in oben gezeigte tibiale Interferenzschraube ergibt sich eine prozentuale Abweichung der Messung von gegebener Schraubenlänge von: $\%=100 \% \times 2,94 / 3,00=2,0 \%$.

Entsprechend ist die prozentuale Abweichung der Messung von gegebenem Schraubendurchmesser: $\%=100 \% \times 0,93 / 0,90=3,3 \%$.

\subsection{Nachuntersuchung}

Die Patienten wurden zwölf Monate nach Erhalt der VKB-Plastik anhand der Kniegelenkfunktionsscores untersucht und befragt. Der Tegner Score, der Lysholm Score, der Marshall Score, der IKDC-Score, die VAS Schmerz und Funktion sowie die Ergebnisse aus der Messung mit dem KT 1000 Arthrometer wurden bei allen zur Nachuntersuchung erscheinenden Patienten zwölf Monate nach Erhalt der VKB-Plastik ermittelt.

\subsection{Statistische Auswertung}

Da eine Normalverteilung der Daten nicht angenommen werden konnte, wurde zur Analyse von Unterschieden zwischen der ST-Gruppe und der STG-Gruppe der Mann-Whitney-U-Rangsummentest verwendet. Dieser Test wurde zum Vergleich der Gruppen für folgende nichtparametrische Daten genutzt: Nachuntersuchung in Monaten, Zeitdauer zwischen Trauma und Operation in Wochen, Tegner Score, Lysholm Score, Marshall Score, IKDC-Score, VAS Schmerz, VAS Funktion, KT 1000 Arthrometer.

Für parametrische Daten (Patientenalter, Body-Mass-Index) wurde der T-Test für unverbundene Stichproben verwendet. Der chi-Quadrat-Test wurde zum Vergleich der Geschlechtsverteilung zwischen den Untersuchungsgruppen genutzt. Die Analyse wurde mit Statistica 8.o durchgeführt. Die femorale und tibiale Volumenabnahme der Schrauben wurde mit dem T-Test für verbundene Stichproben verglichen. Die Analyse erfolgt mit Statistica 8.0.

Das Tunnelenlargement wurde mit einer mehrfachen Varianzanalyse für longitudinale Daten analysiert. Im Falle eines signifikanten Ergebnisses erfolgte ein Alpha Abgleich mittels Bonferroni-Holm. Die Analsyse des Tunnelenlargements erfolgte mit SAS 9.1 ( SAS Institute Inc., Cary, NC, USA). Als signifikant wurde ein Ergebnis bezeichnet, wenn die Irrtumswahrscheinlichkeit p kleiner als 0,05 war. 


\section{Ergebnisse}

\subsection{Patientenkollektiv}

Von den ursprünglich 37 Patienten, deren VKB-Ersatzplastik femoral und tibial mit Milagro ${ }^{\circledR}$ Interferenzschrauben fixiert wurde, konnten von 35 Patienten alle erforderlichen Daten erhoben werden.

Die an der vorliegenden Studie beteiligten Patienten waren zwischen 16 und 44 Jahre alt. Das durchschnittliche Alter der Patienten des Gesamtkollektivs betrug 25,4 Jahre (+/- 6,7 Jahre). Die meisten Patienten waren zwischen 21 und 30 Jahre alt.

\begin{tabular}{|c|cr|rr|rrr|}
\hline $\begin{array}{c}\text { Alter } \\
\text { [Jahre] }\end{array}$ & abs. & Fraven & Männer & Gesamt & abs. \\
\hline$<21$ & 2 & 5,7 & 4 & 11,4 & 6 & 17,1 \\
\hline $21-30$ & 10 & 28,5 & 12 & 34,3 & 22 & 62,8 \\
\hline $31-40$ & 3 & 8,6 & 2 & 5,7 & 5 & 14,3 \\
\hline$>40$ & 1 & 2,9 & 1 & 2,9 & 2 & 5,8 \\
\hline
\end{tabular}

Tabelle 1: Altersverteilung des Patientenkollektivs

Der mittlere Body-Mass-Index (BMI) des Gesamtkollektivs lag bei $24,8 \mathrm{~kg} / \mathrm{m}^{2}$. Im Durchschnitt wurden die Patienten 13,0 Monate nach der Operation nachuntersucht.

Kein Patient des Gesamtkollektivs erhielt eine VKB-Ersatzplastik in der akuten Phase. Die Patienten wurden durchschnittlich 15,2 Wochen nach dem Trauma operiert.

\begin{tabular}{|l|c|}
\hline \multicolumn{1}{|c|}{ Gruppe } & Transplantatfixation mit Milagro ${ }^{\circledR}$-Interferenzschrauben \\
\hline BMI (kg/m²) & $24,8(+/-4,5 ; 18-41,5)$ \\
\hline Nachuntersuchung (Monate) & $13,0(+/-1,6 ; 11-18)$ \\
\hline Intervall Trauma - OP (Wochen) & $15,2(+/-34,4 ; 3-200)$ \\
\hline Anzahl akut (\%) & $0(0 \%)$ \\
\hline Anzahl subakut (\%) & $27(77,1 \%)$ \\
\hline Anzahl chronisch (\%) & $8(22,9 \%)$ \\
\hline
\end{tabular}

Tabelle 2: Patientendaten: Mittelwert ( \pm Standardabweichung; Range) 


\subsection{Verletzungsursachen}

Die Sportarten mit azyklischen Bewegungsabläufen und möglichen Fremdeinwirkungen durch Gegenspieler stellten den weitaus größten Anteil des Patientengutes dar.

25 Patienten zogen sich in vorliegender Studie die VKB-Ruptur bei einer High-Risk-Pivoting Sportart zu, davon betrieben alleine 20 Patienten die Sportarten Fussball und Handball. Sechs Patienten zogen sich die VKB-Ruptur bei Low-Risk-Pivoting Sportarten wie Eislaufen oder Inline Skaten zu. An der Nachuntersuchung nahm kein Patient teil, der sich die VKB-Ruptur bei einer Non-Risk-Pivoting Sportart zuzog. Vier Patienten erlitten eine VKB-Ruptur bei Freizeitaktivitäten wie Fahrrad fahren und wurden unter Sonstiges eingeteilt.

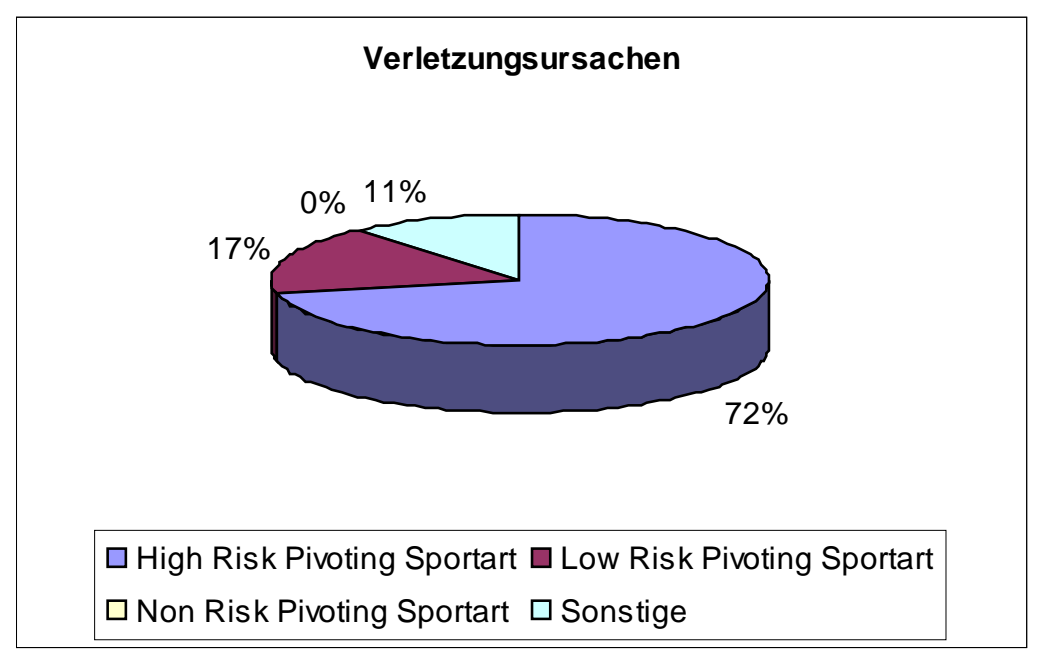

Abbildung 9: Verletzungsursachen für die VKB-Ruptur

\subsection{Klinische Ergebnisse bei Transplantatfixation mit Milagro ${ }^{\circledR}$ Interferenzschrauben}

\subsubsection{Tegner Aktivitätsskala}

Der mittlere präoperative Tegner Score lag bei den 35 zur Nachuntersuchung erschienenen Patienten bei $6,97(+/-1,68 ; 3-9)$. Bei Transplantatfixation der VKB-Plastik mittels femoraler und tibialer Milagro ${ }^{\circledR}{ }_{-}$ Interferenzschraube lag der mittlere postoperative Tegner Score nach einem Jahr bei $6,17(+/-1,98 ; 3-9)$. 21 (60\%) der 35 Patienten erreichten nach einem Jahr den gleichen Aktivitätslevel wie vor der Operation. Fünf Patienten (14,3\%) berichten von einer Aktivitätsabnahme von einem Punkt, sechs Patienten $(17,1 \%)$ von einer Aktivitätsabnahme von zwei Punkten, zwei Patienten $(5,7 \%)$ von einer 
Aktivitätsabnahme von drei Punkten und ein Patient $(2,9 \%)$ von einer Aktivitätsabnahme von fünf Punkten.

\subsubsection{Lysholm Score}

Ein Jahr postoperativ wurde bei den 35 zur Nachuntersuchung erschienenen Patienten ein mittlerer Lysholm Score von 93,4 (+/- 5,4; 80-100) erreicht. Bei 17 Patienten konnte ein Lysholm Score von größer 95 Punkten und damit ein exzellentes Ergebnis erzielt werden. ${ }_{15}$ Patienten erreichten im Lysholm Score ein gutes und drei Patienten ein zufriedenstellendes Ergebnis. Kein Patient erreichte bei Transplantatfixation mit Milagro ${ }^{\circledR}$-Schrauben ein schlechtes Ergebnis im Lysholm Score.

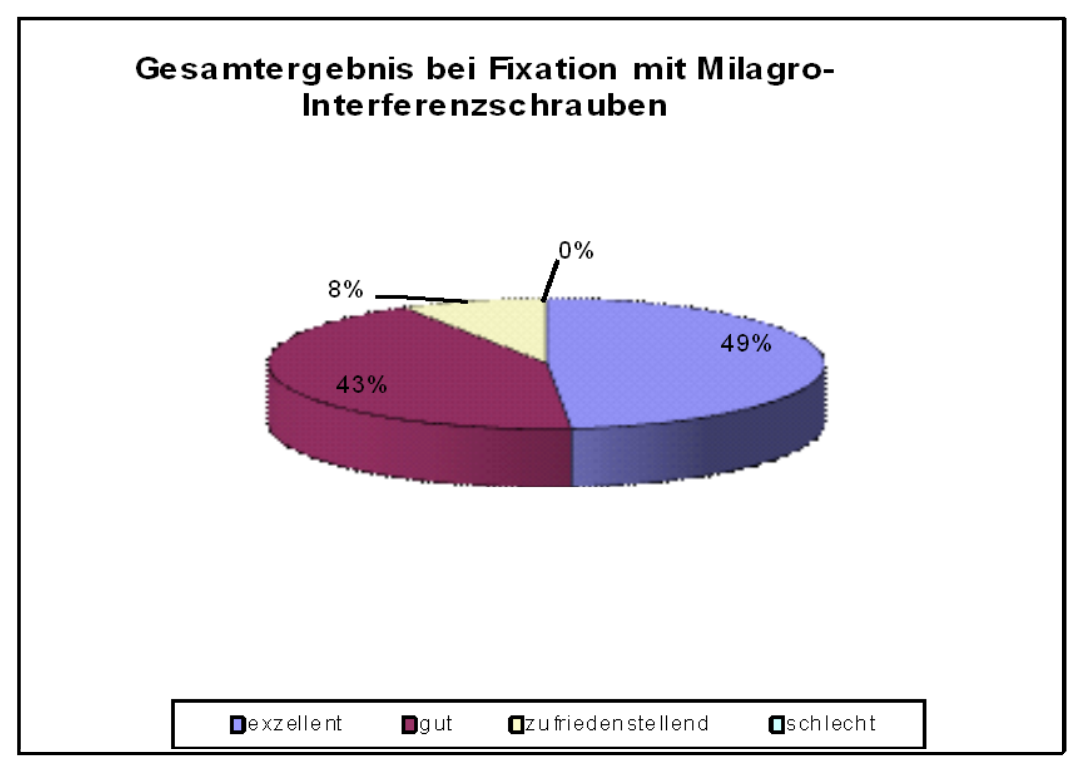

Abbildung 10: Gesamtergebnis des Lysholm Scores

Drei Patienten beurteilten ihre Kniegelenksfunktion als zufriedenstellend. Ein 22 jähriger Patient erreichte 8o Punkte. Er gab Instabilitätsgefühl bei Belastung an, verstärkte Schmerzen bei Extrembelastungen und hatte eine Oberschenkelatrophie von mehr als $2 \mathrm{~cm}$.

Ein 32 jähriger Mann, der 81 Punkte erreichte berichtete über leichte Schmerzen beim Treppensteigen sowie bei Extrembelastung, hatte eine Oberschenkelatrophie von größer als $2 \mathrm{~cm}$ und gab ein Instabilitätsgefühl bei Belastung an. Eine 38 jährige Patientin erreichte 82 Punkte und berichtete über Schmerzen und Schwellneigung bei normaler Belastung.

\subsubsection{Marshall Score}

Bei Transplantatfixation mit Milagro ${ }^{\circledR}$-Schrauben lag der mittlere Marshall Score ein Jahr nach Erhalt des VKB-Ersatzes bei 46,6 Punkten (+/- 2,5; 39-50). Von den 35 an der Nachuntersuchung 
teilnehmenden Patienten konnte bei 34 Patienten ein Marshall Score von größer 41 Punkten und damit ein sehr gutes Ergebnis erzielt werden. Eine Patientin erreichte einen Marshall Score von 39 Punkten und damit ein gutes Ergebnis.

\subsubsection{Visuelle Analogskala Schmerz und Funktion}

Bei Transplantatfixation mit der bioresorbierbaren Interferenzschraube Milagro ${ }^{\circledR}$ gaben die Patienten ein Jahr nach Ersatz des VKB einen mittleren Wert von 1,2 (+/- 1,4; 0-5) auf der VAS Schmerz an. 15 Patienten gaben auf der VAS Schmerz eine $O$ und neun Patienten gaben eine 1 an. Fünf Patienten gaben eine 2, drei Patienten eine 3, zwei Patienten eine 4 und ein Patient gab auf der VAS Schmerz eine 5 an.

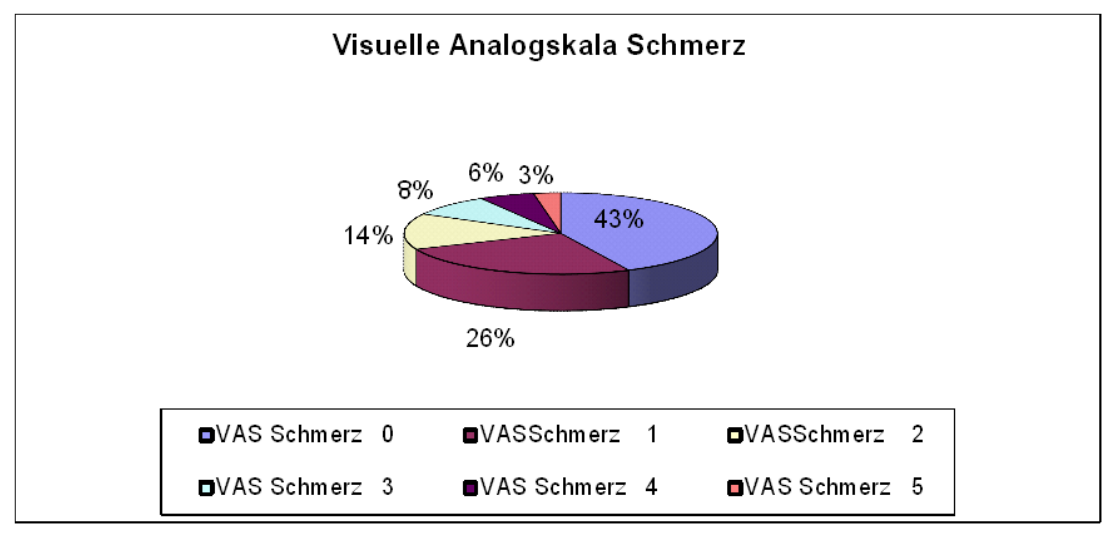

Abbildung 11: Prozentuale Verteilung VAS Schmerz

Auf der VAS Funktion ergab sich beim Gesamtkollektiv ein mittlerer Wert von 9,0 (+/- 1,0; 7-10). 13 Patienten gaben eine 10 und zwölf Patienten gaben eine 9 an. Sechs Patienten gaben eine 8 und vier Patienten eine 7 an.

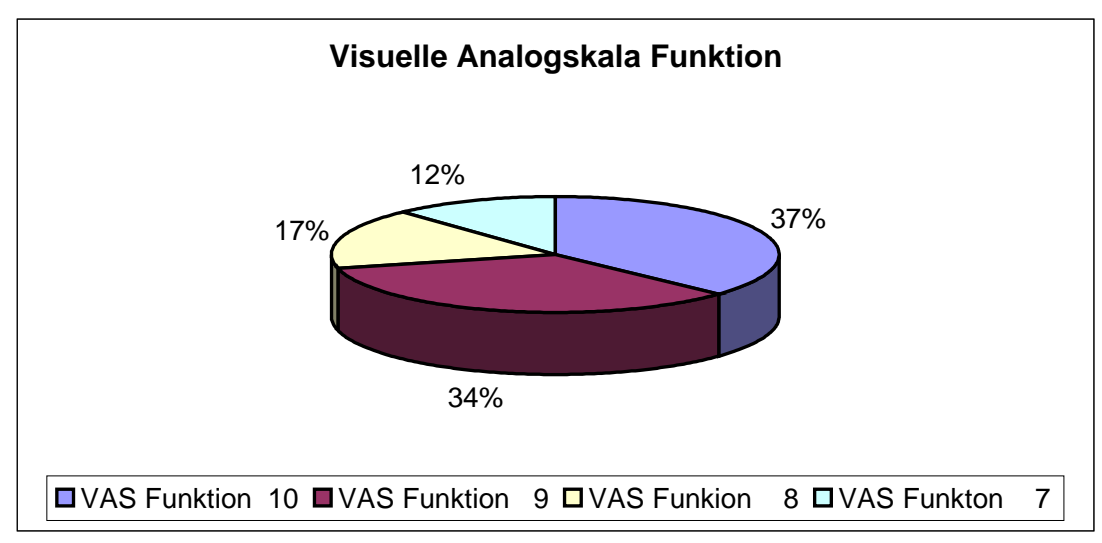

Abbildung 12: Prozentuale Verteilung VAS Funktion 


\subsubsection{IKDC-Evaluationsblatt}

\subsubsection{Subjektive Beurteilung}

Bei Transplantatfixation mit Milagro ${ }^{\circledR}$-Interferenzschrauben beurteilten 17 Patienten (49\%) die Funktionalität ihres Kniegelenkes mit A als „normal”. 18 Patienten (51\%) beantworteten die Frage „Wie funktioniert Ihr Knie?" mit „fast normal" und wurden als B eingestuft.

\subsubsection{Rate optimaler Operationsergebnisse}

Von den 35 zur Nachuntersuchung erschienenen Patienten gaben 18 Patienten $(51,4 \%)$ an, keine Beschwerden bei belastenden Rotationen oder Kontaktsportarten zu haben. Sie wurden als "normal" (A) eingestuft. Elf Patienten (31,4\%) gaben an, keine Beschwerden bei schwerer körperlicher Arbeit oder Tennis zu haben. Ihre Einstufung erfolgte unter B als „fast normal”. Sechs Patienten (17,1\%) gaben an, keine Beschwerden bei leichter körperlicher Arbeit, Joggen oder Schwimmen zu haben. Sie wurden als „abnormal" (C) eingestuft. Kein Patient aus dem Gesamtkollektiv wurde mit D gruppiert.

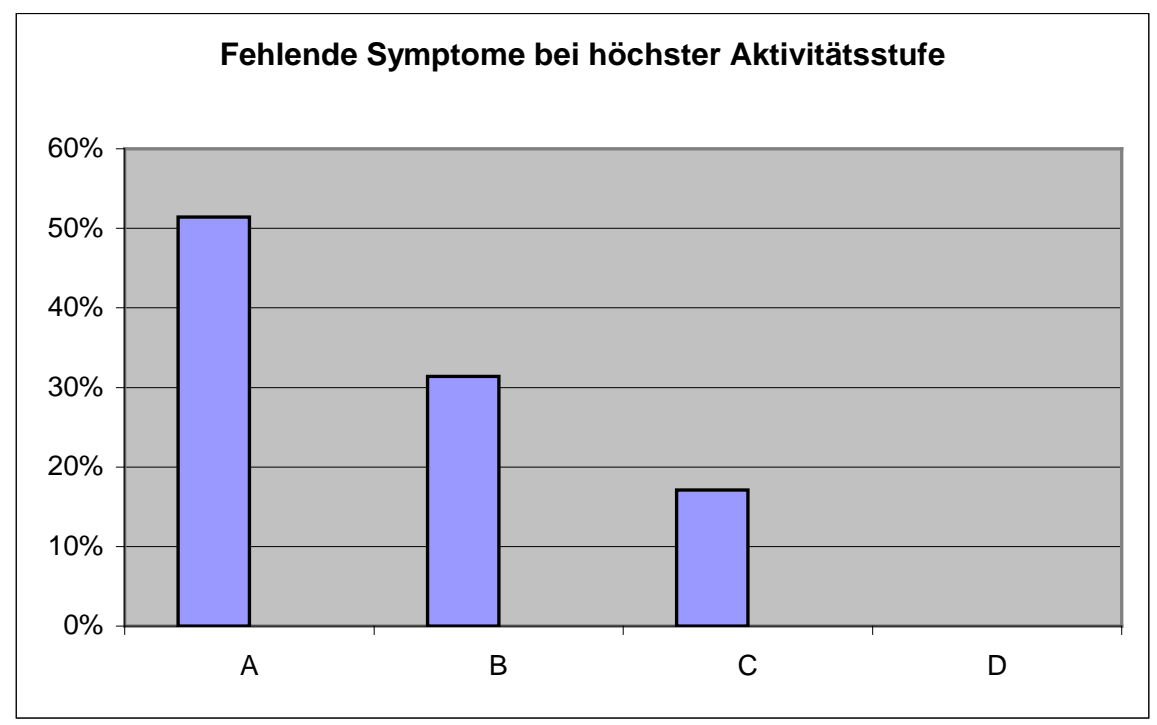

Abbildung 13: IKDC-Score - Fehlende Symptome bei höchster Aktivitätsstufe

\subsubsection{Objektive Beurteilung}

Im Gesamtkollektiv hatten 22 Patienten keine vermehrte anteriore Translation im Lachman-Test und wurden mit „normal" (A) eingestuft, fünf Patienten wurden bei einfach positivem Lachman-Test mit „fast normal" (B) eingestuft. 8 Patienten wurden bei zweifach positivem Lachman-Test mit "abnormal" (C) bewertet. Bei keinem Patienten, bei dem die Transplantatfixation mit Milagro ${ }^{\circledR}$ Interferenzschrauben erfolgte fand sich ein weicher Anschlag mit dreifach positivem Lachman-Test. 


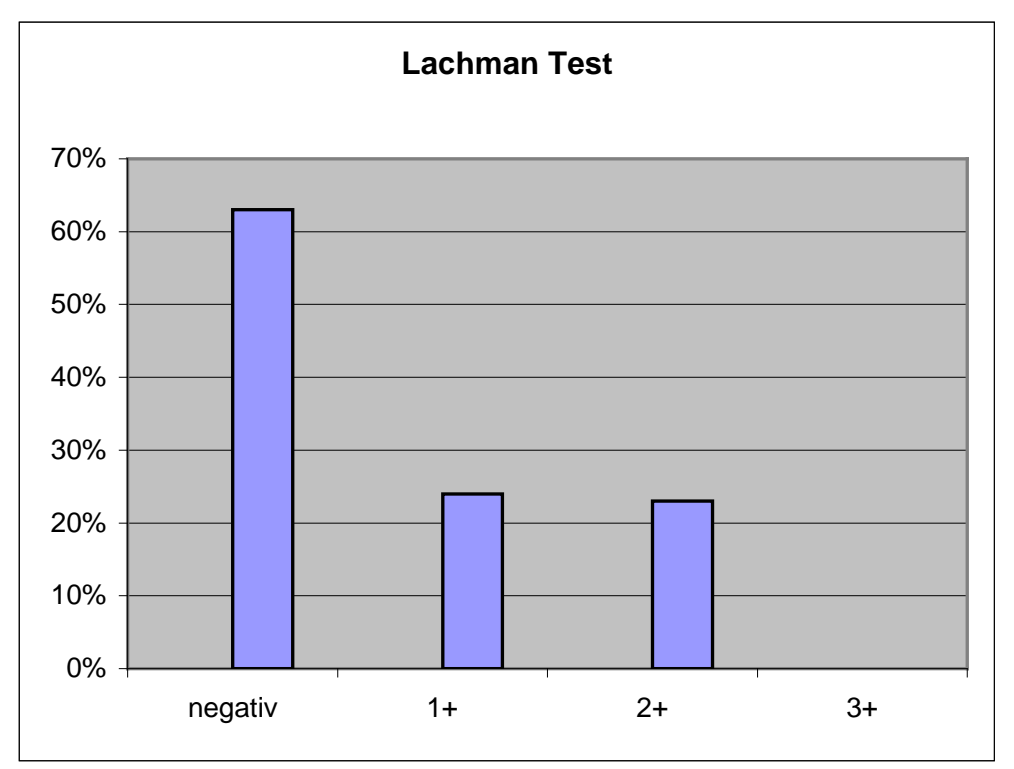

Abbildung 14: IKDC-Score: Lachman-Test in $25^{\circ}$ Flexion

\section{3·3.5.4 Symptome an der Transplantatentnahmestelle}

Im Gesamtkollektiv gaben 24 Patienten (68,6\%) keine Symptome an der Transplantatentnahmestelle an und wurden mit "normal” (A) gewertet. Zehn Patienten (28,6\%) gaben geringe Hypästhesien an der Transplantatentnahmestelle an und wurden mit "fast normal" (B) eingestuft. Eine Patientin (2,9\%) wurde bei Hypästhesien an der Transplantatentnahmestelle von größer als $20 \mathrm{~cm}^{2}$ mit $C$ bewertet.

\subsubsection{Gesamtbeurteilung}

Vom Gesamtkollektiv wurden elf Patienten (31\%) in der Gesamtbeurteilung mit A als „"normales" Kniegelenk eingestuft, 13 Patienten (37\%) wurden mit B als "fast normal" eingestuft, zehn Patienten (29\%) wurden mit C als „abnormal" eingestuft und ein Patient (3\%) wurde mit D als "stark abnormal” eingestuft.

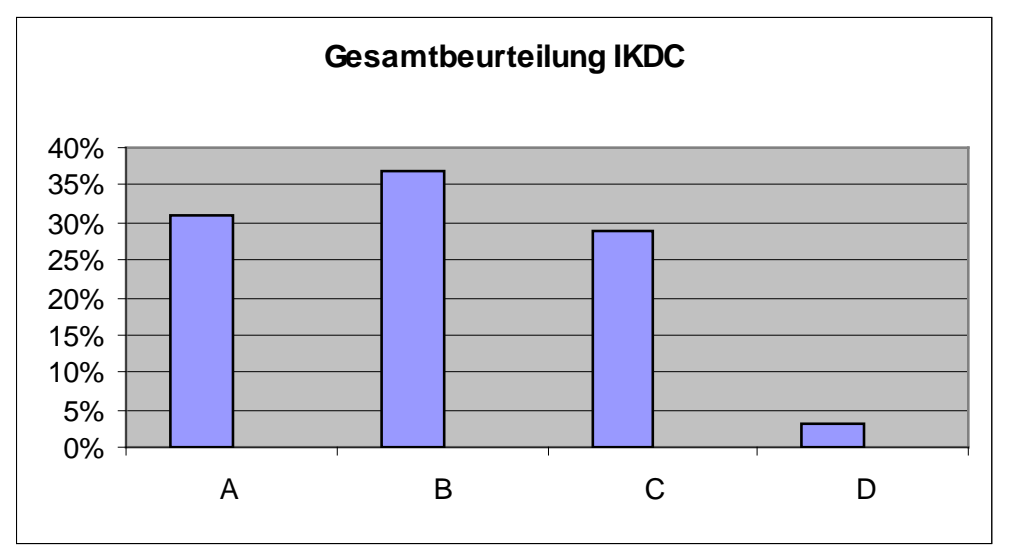

Abbildung 15: IKDC-Score - Gesamtbeurteilung 
3.4 Vergleich des klinischen Ergebnisses bei VKB-Rekonstruktion mit der

Semitendinosussehne alleine versus Semitendinosus- und

\author{
Gracilissehne als Transplantat
}

\title{
3.4.1. Kniegelenkfunktionsscores
}

Von den 35 Studienteilnehmern wurde bei 15 Patienten als VKB-Ersatztransplantat die Semitendinosussehne alleine und bei 20 Patienten die Semitendinosus- und Gracilissehne verwendet. Das durchschnittliche Alter der Patienten in der Gruppe "Semitendinosus" betrug 23,7 Jahre, in der Gruppe "Semitendinosus und Gracilis" waren die Patienten durchschnittlich 26,6 Jahre alt. Der mittlere Body-Mass-Index (BMI) der Gruppe "Semitendinosus" lag bei 25,5 und in der Gruppe "Semitendinosus und Gracilis" bei 24,3. Hinsichtlich der Geschlechtsverteilung $(p=0,50)$, des Alters $(p=0,21)$ und des BMI $(p=0,44)$ fanden sich keine signifikanten Unterschiede zwischen den Untersuchungsgruppen. Die Zeitdauer bis zur Nachuntersuchung ergab keine signifikanten Unterschiede zwischen den Untersuchungsgruppen $(p=0,57)$.

Bei den 15 Patienten, deren Kreuzbandersatztransplantat aus der Semitendinosussehne alleine besteht, fällt im Tegner Score der mittlere Aktivitätslevel von 7,0 (+/- 2,0; 3-9) präoperativ auf einen Wert von 6,6 (+/- 2,2; 3-9) postoperativ. In der Gruppe "Semitendinosus und Gracilis" fällt der mittlere Aktivitätslevel von 6,95 (+/- 1,5;4-9) auf 5,85 (+/- 1,9; 3 - 9). Beim Vergleich des präopertiven Tegner Scores gab es zwischen der Gruppe "Semitendinosus" und der Gruppe "Semitendinosus und Gracilis" keine signifikanten Unterschiede $(p=0,88)$. Bei den postoperativen Ergebnissen konnten ebenfalls keine signifikanten Unterschiede zwischen den Untersuchungsgruppen festgestellt werden $(p=0,29)$. Im Lysholm Score ergab sich in der Gruppe „Semitendinosus" durchschnittlich ein Wert von 94,5 (+/- 5,1; 80-100) und in der Gruppe „Semitendinosus und Gracilis" von 92,6 (+/- 5,8; 81-100) Punkten. Zwischen den Untersuchungsgruppen gab es im Lysholm Score keine signifikanten Unterschiede $(p=0,26)$.

In der Gruppe "Semitendinosus" lag der mittlere Marshall Score bei 47,7 (+/- 2,1; 43-50) und in der Gruppe „Semitendinosus und Gracilis" bei 45,8 (+/- 2,5; 39-50) Punkten. Für die „Gruppe Semitendinosus" konnte dabei ein signifikant besseres Ergebnis im Marshall Score $(p=0,037)$ nachgewiesen werden.

In der Gruppe "Semitendinosus" fand sich auf der VAS Schmerz ein mittlerer Wert von 1,3 (+/- 1,4;0-4) und in der Gruppe „Semitendinosus und Gracilis" von 1,2 (+/- 1,4; 0-5). Die subjektiv empfundene Funktionalität des operierten Kniegelenks lag in der Gruppe „Semitendinosus" bei 9,1 (+/-1,0; 7-10) und in der Gruppe „Semitendinosus und Gracilis" bei 8,9 (+/-1,1; 8-10). Sowohl für die VAS Schmerz $(p=0,65)$ 
als auch für die VAS Funktion $(p=0,70)$ ergaben sich keine signifikanten Unterschiede $z$ wischen der Gruppe "Semitendinosus" und der Gruppe "Semitendinosus und Gracilis".

\subsubsection{Instrumentelle Stabilitätsprüfung mit dem KT 1000 Arthrometer}

Die mittlere Seitendifferenz lag bei Transplantatfixation mit der Milagro ${ }^{\circledR}$-Schraube bei einer Zugkraft von 67 Newton bei 1,61 mm, bei einer Zugkraft von 89 Newton bei 1,94 $\mathrm{mm}$ und beim MMD bei 2,19 $\mathrm{mm}$. In Tabelle 3 sind die mittleren Seitendifferenzen in $\mathrm{mm}$ zwischen dem operierten und nicht operierten Knie für das Gesamtkollektiv, die Gruppe „Semitendinosus" und die Gruppe „Semitendinosus und Gracilis" dargestellt. Mit zunehmender Zugkraft nimmt die anteriore Translation des operierten Kniegelenks im Vergleich zur Gegenseite zu.

\begin{tabular}{|c|cc|cc|cc|}
\hline \multicolumn{1}{|c|}{ Ventrale Translation } & \multicolumn{2}{|c|}{67 Newton } & \multicolumn{2}{|c|}{89 Newton } & \multicolumn{2}{c|}{ MMD } \\
\hline Gruppe & & & & & & \\
\hline "ST" 12 Monate postperativ & 1,61 & $(+/-1,1 ; 0-4)$ & 1,94 & $(+/-1,1 ; 0-5)$ & 2,19 & $(+/-1,3 ; 0-5)$ \\
\hline "STG" 12 Monate postoperativ & 1,50 & $(+/-1,0 ; 0-3,5)$ & 1,93 & $(+/-1,3 ; 0-4)$ & 2,40 & $(+/-1,6 ; 0-5)$ \\
\hline
\end{tabular}

Tabelle 3: Seitendifferenz in mm mit dem KT 1000 Arthrometer (+/- Standardabweichung; Range)
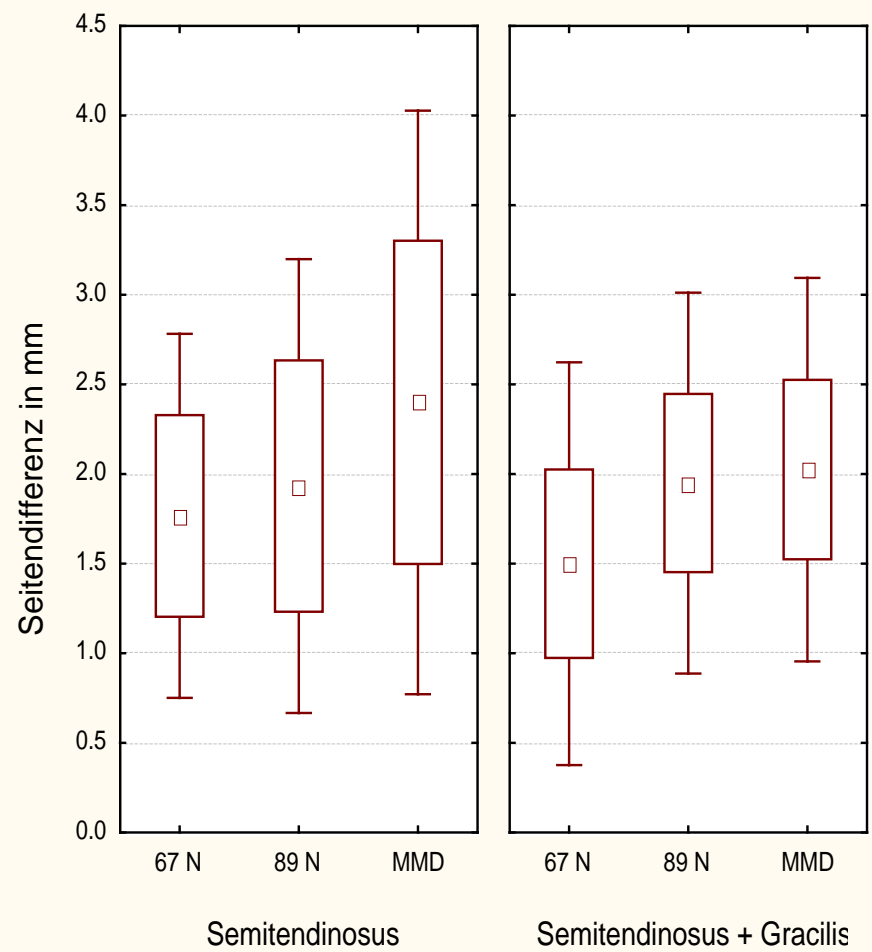

$\square$ Mittelwert

Mittelwert+0.95 Konf. Interv

I Mittelwert+Stdabw.

Abbildung 16: Seitendifferenz $\Delta$ in mm im KT 1000 Arthrometer 
Zwischen der Gruppe "Semitendinosus" und der Gruppe "Semitendinosus und Gracilis" kam es dabei nicht zu signifikanten Unterschieden. Bei einer Zugkraft von $67 \mathrm{~N}(p=0,43), 89 N(p=0,85)$ und beim MMD $(p=0,65)$ lag der $p$-Wert über dem Signifikanzniveau von 0,05 (Abbildung 16).

\subsection{Komplikationen}

Eine Patientin musste postoperativ wegen einer tiefen Beinvenenthrombose behandelt werden. In der Nachuntersuchungsgruppe kam es bei keinem Patienten zu postoperativen Infekten.

Bei vier von 35 Patienten $(11,4 \%)$ war bis zum Zeitpunkt der Nachuntersuchung eine Rearthroskopie nötig.

Bei einem Patienten fand sich arthroskopisch ein nicht verheilter Innenmeniskusriss nach Naht, bei einer Patientin resistierende Schmerzen bei einem Knorpelschaden III.-IV. Grades nach ICRS und bei zwei Patienten ein Zyklops-Tumor.

\subsection{Milagro ${ }^{\circledR}$-Interferenzschrauben im konventionellen Röntgen}

Bei allen Patienten wurde postoperativ eine Röntgenaufnahme des Kniegelenks in 2 Ebenen angefertigt. Dabei konnte die Lage der Bohrkanäle für das Transplantat kontrolliert werden. Da die Milagro ${ }^{\circledR}$-Schrauben röntgendicht sind, konnte die Lage der Schrauben im konventionellen Röntgen evaluiert werden (Abbildung 17). Bei allen an der Studie teilnehmenden Patienten konnten auf diese Weise Schraubenfehlplatzierungen oder Dislokationen ausgeschlossen werden.
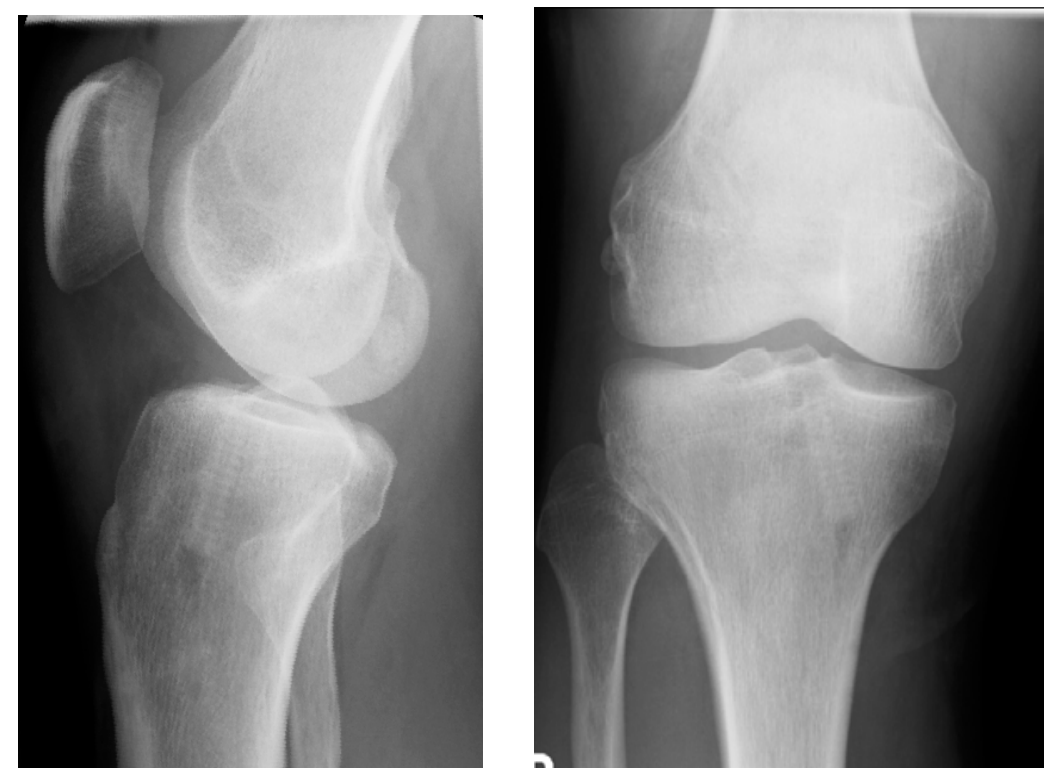

Abbildung 17 aus Frosch et al. (2009, S.75): Milagro ${ }^{\circledR}$-Interferenzschraube im konventionellen Röntgenbild (Mit freundlicher Genehmigung des Springer Verlages). 


\subsection{Kernspintomographische Ergebnisse}

\subsubsection{Messgenauigkeit in der 3D-DESS Sequenz}

Bei acht direkt postoperativ durchgeführten MRT-Untersuchungen des operierten Kniegelenkes wurde die Messgenauigkeit in der 3D-DESS Sequenz für die tibialen Milagro ${ }^{\circledR}$-Schrauben ermittelt. Die mittlere prozentuale und absolute Abweichung der Messung von den tatsächlichen Schraubenmaßen zeigt Tabelle 4.

Die mittlere absolute Abweichung in $\mathrm{mm}$ betrug für die tibiale Schraubenlänge $0,75 \mathrm{~mm}$ und für den tibialen Schraubendurchmesser 0,29 mm.

\begin{tabular}{|c|c|c|c|}
\hline \multicolumn{2}{|c|}{ Abweichung Schraubenlänge } & \multicolumn{2}{c|}{ Abweichung Schraubendurchmesser } \\
\hline prozentual & absolut & prozentual & absolut \\
\hline $2,57 \%$ & $0,75 \mathrm{~mm}$ & $3,45 \%$ & $0,29 \mathrm{~mm}$ \\
$(+/-1,9 ; 0,43-6,6)$ & $(+/-0,5 ; 0,1-1)$ & $(+/-2,3 ; 0-7,5)$ & $(+/-0 ; 0-0,6)$ \\
\hline
\end{tabular}

Tabelle 4: Abweichung der Messung im MRT: Arithmetisches Mittel (+/- Standardabweichung; Range)

\subsubsection{Milagro ${ }^{\circledR}$-Interferenzschraube in der Kernspintomographie}

Morphologisch stellten sich die Schrauben im MRT als negativer Kontrast zum Knochen und Weichteilgewebe eindeutig dar und waren leicht zu vermessen (Abbildung 8). Nur direkt postoperativ und nach drei Monaten fand sich ein deutliches Knochenmarksödem im Bereich der Bohrkanäle, welches nach sechs Monaten bereits vollständig verschwunden war. Flüssigkeitssäume um die Schrauben konnten zu keinem Zeitpunkt festgestellt werden.

Bei einer Patientin fand sich am tibialen Transplantatansatz eine zystische Struktur jedoch ohne klinische Beschwerden und ohne Hinweise auf Fremdkörperreaktion. Die Schraube selbst erreichte die Zyste nicht.

Das Transplantat konnte ebenfalls bei allen Patienten identifiziert werden, es zeigte sich bei allen Patienten eine regelrechte Transplantatlage, bei einem Patienten war die dorsale femorale Kanalwand ausgebrochen, klinisch zeigte sich bei diesem Patienten eine stabile Transplantatheilung ohne Beschwerden. Das Transplantat konnte jeweils auf einer Länge von mindestens 1,5 cm durch die Schrauben erfasst werden.

Bei allen Patienten kam es im Verlauf zum Einwachsen von Knochengewebe in die sich abbauenden Schrauben. 


\subsubsection{Resorption der Milagro ${ }^{\circledR}$-Interferenzschrauben}

Im arithmetischen Mittel lag bei den tibialen Milagro ${ }^{\circledR}$-Interferenzschrauben der Volumenverlust nach sechs Monaten bei 8,1 \% (+/- 7,9; 4-26,7 \%) und nach zwölf Monaten bei 82,57 \% (+/- 17,2; 46,6-100\%). Während bei den tibialen Interferenzschrauben der durchschnittliche Volumenverlust nach sechs Monaten bei $8,1 \%(+/-7,9 ; 4-26,7 \%)$ lag, ergab sich femoral zum selben Zeitpunkt ein durchschnittlicher Volumenverlust von 31,26\% (+/- 21,6;3,4-74,2\%). Zwölf Monate postoperativ ergab sich femoral ein durchschnittlicher Volumenverlust von $92,02 \%(+/-6,0 ; 78,7-100 \%)$.

Der prozentuale Volumenverlust der tibialen versus femoralen Interferenzschraube ist in Abbildung 18 dargestellt.

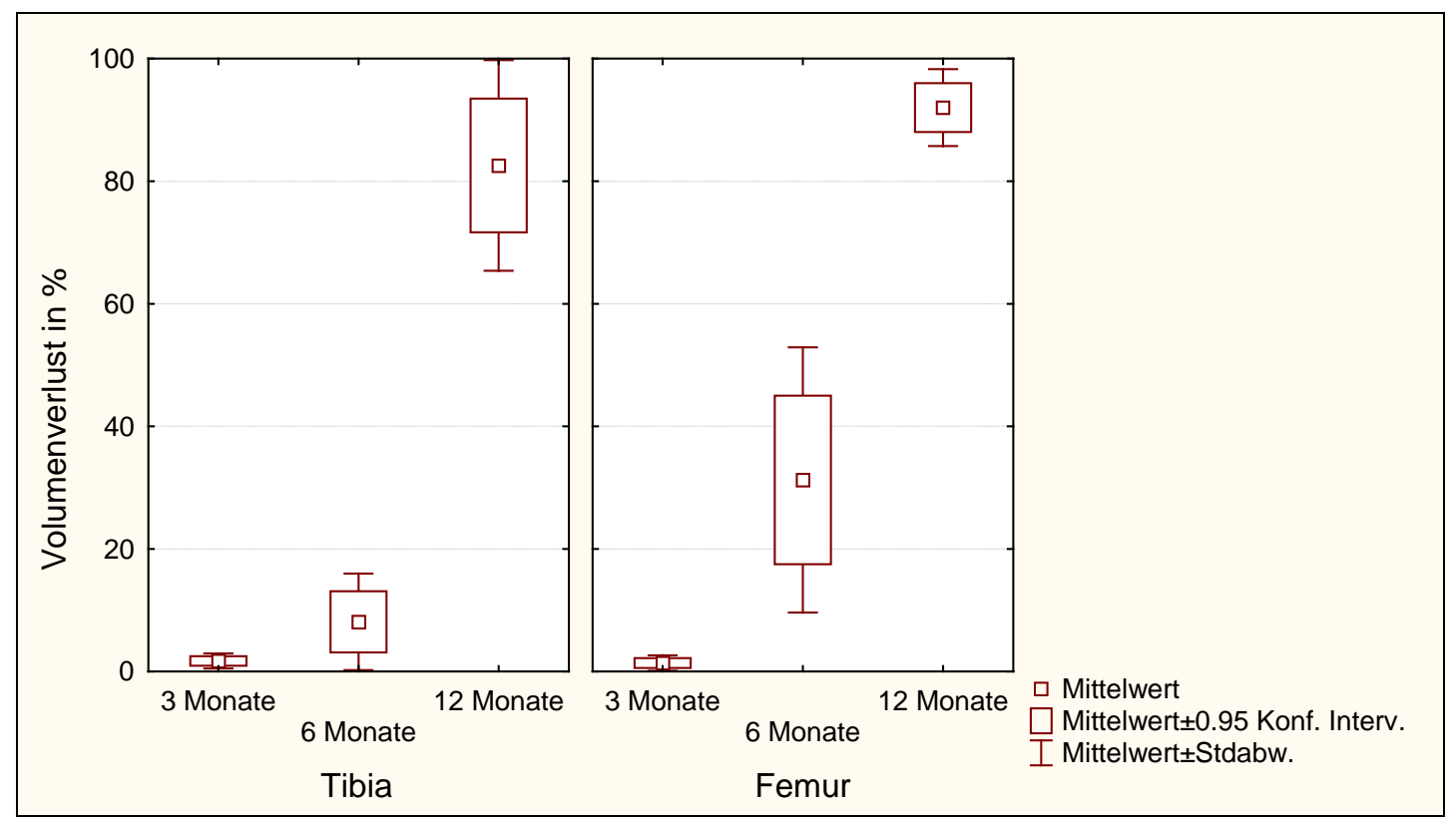

Abbildung 18: Tibialer und femoraler Volumenverlust der Milagro ${ }^{\circledR}$-Schraube

Die Abbildung zeigt, dass femoral die Schraube schneller bioabsorbiert wird als tibial.

Drei $(p=0,43)$ und zwölf Monate $(p=0,59)$ postoperativ ergaben sich keine signifikanten Unterschiede zwischen femoralem und tibialem Volumenverlust der Schrauben. Nach sechs Monaten war die Volumenabnahme femoral signifikant höher als bei den tibialen Interferenzschrauben $(p=0,035)$. Bei keinem Patienten der Studie konnte drei Monate nach der Operation ein relevanter Abbau der tibialen Interferenzschraube festgestellt werden. 


\subsubsection{Tibiale Milagro ${ }^{\circledR}$-Interferenzschraube}

In Abbildung 19 a ist eine tibial gelegene Milagro ${ }^{\circledR}-$ Interferenzschraube mit einer primären Länge $I_{t}$ von $23 \mathrm{~mm}$ und einem primären Durchmesser $\mathrm{d}_{\mathrm{g}}$ von $8 \mathrm{~mm}$ drei Monate nach Ersatz des vorderen

Kreuzbandes dargestellt.

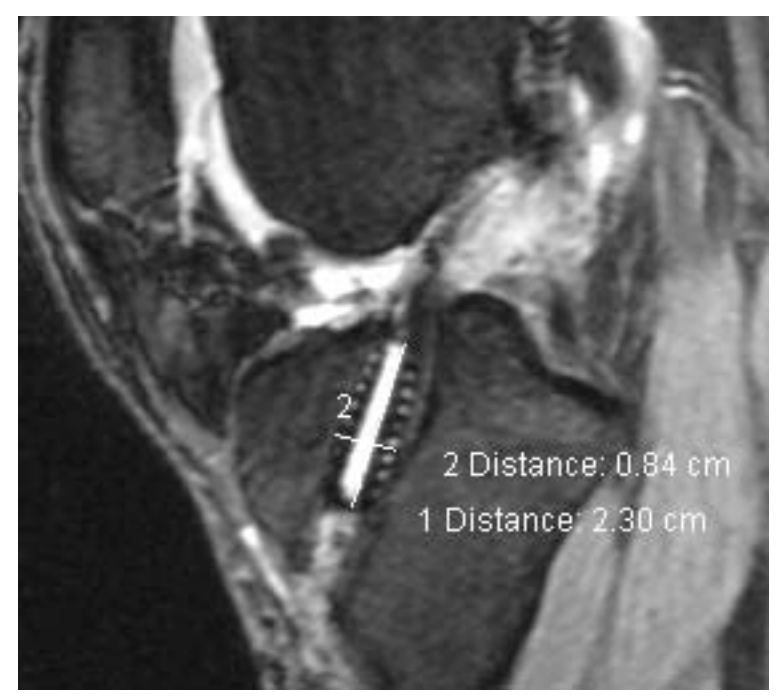

Abbildung 19a: Milagro ${ }^{\circledR}$-Interferenzschraube tibial drei Monate nach VKB-Ersatz.

Im Bereich der Bohrkanäle können keine Hinweise auf Ödembildungen oder Osteolysen festgestellt werden. Ebenfalls zeigt sich drei Monate postoperativ kein Flüssigkeitssaum um die Schrauben. Eine messbare Resorption der Interferenzschraube hat noch nicht stattgefunden.

Eine Milagro ${ }^{\circledR}$-Interferenzschraube $(9 \mathrm{~mm} \times 23 \mathrm{~mm}$ ) ist sechs (Abbildung $19 \mathrm{~b}$ ) und zwölf Monate (Abbildung 19 c) nach Ersatz des vorderen Kreuzbandes dargestellt.
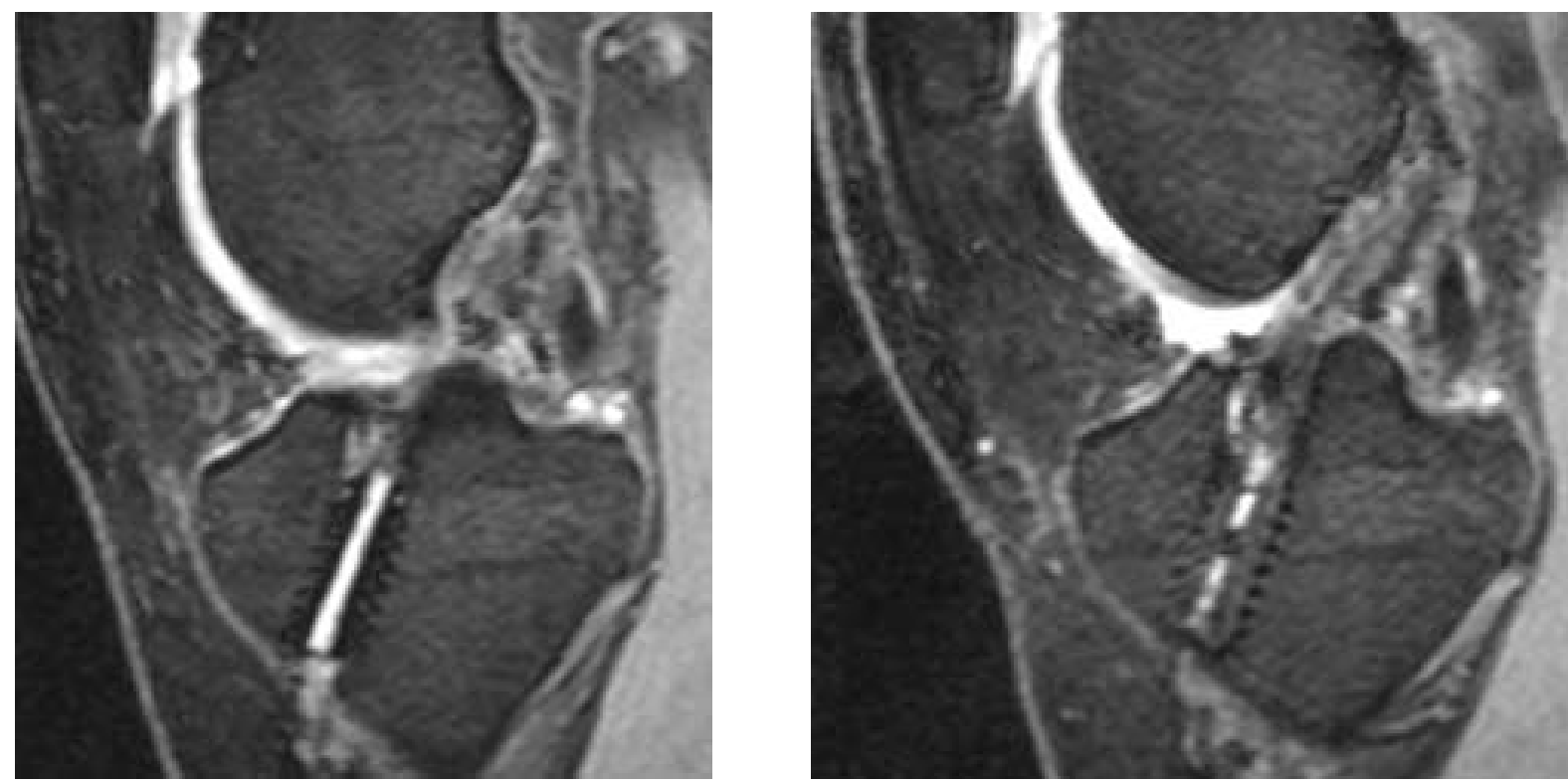

Abbildung 19 b und c aus Frosch et al. (2009, S.76): MRT Knie einer 23 jährigen weiblichen Patientin sechs und zwölf Monate nach VKB-Ersatz (mit freundlicher Genehmigung des Springer Verlages).

Kernspintomographisch zeigt sich, dass die tibiale Interferenzschraube nach sechs Monaten nahezu vollständig erhalten ist und nach zwölf Monaten nur noch Schraubenspuren erkennbar sind. Des Weiteren ist einwachsendes Knochengewebe in die sich abbauende Schraube sichtbar. 


\subsubsection{Femorale Milagro ${ }^{\circledR}$-Interferenzschraube}

Abbildung 20 a zeigt eine femorale Interferenzschraube $(8 \times 23 \mathrm{~mm})$ in der 3D-DESS Sequenz drei Monate nach VKB-Ersatz.

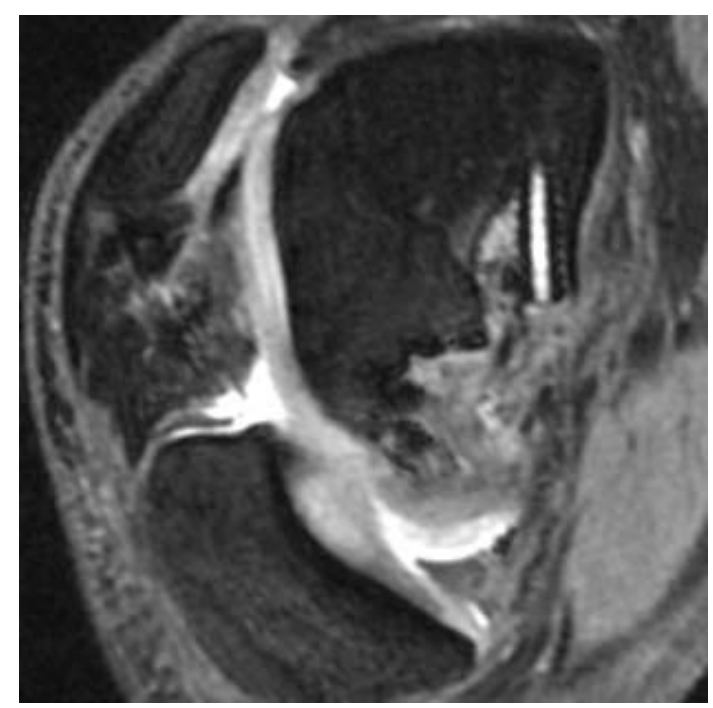

Abbildung 20 a: Milagro ${ }^{\circledR}$-Interferenzschraube femoral drei Monate nach VKB-Ersatzplastik.

Die Milagro ${ }^{\circledR}$-Interferenzschraube ist vollständig erhalten. Eine beginnende Resorption der Schraube ist nicht ersichtlich. Im Bereich der Bohrkanäle zeigen sich keine Hinweise auf Fremdkörperreaktionen.

Die Abbildung $20 \mathrm{~b}$ und c zeigt die gleiche Interferenzschraube sechs und zwölf Monate nach VKB-

Ersatz.
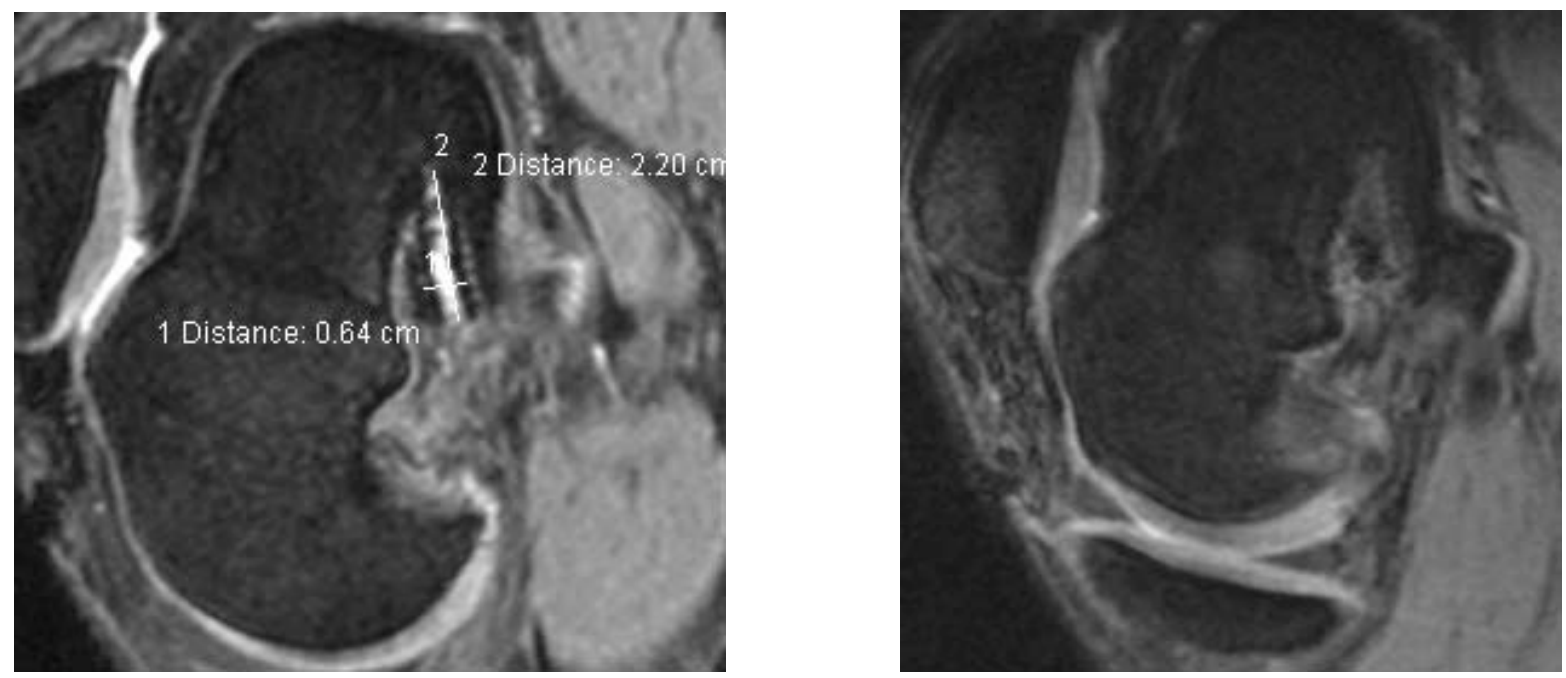

Abbildung 20 b/c: Milagro ${ }^{\circledR}$-Interferenzschraube femoral sechs (b) und zwölf (c) Monate nach VKB-Ersatz. Nach sechs Monaten ist bereits deutlich eine beginnende Resorption zu erkennen.

Nach zwölf Monaten ist die femorale Milagro ${ }^{\circledR}$-Interferenzschraube nahezu vollständig resorbiert. 


\subsubsection{Tunnelenlargement bei Fixation mit Milagro ${ }^{\circledR}$-Interferenzschrauben}

Das durchschnittliche tibiale Tunnelenlargement war $43,5 \%(+/-26,1 ;-2,65-83,27 \%)$ nach drei Monaten, $62,7 \%(+/-30,0 ; 30,2-136,4 \%)$ nach sechs Monaten und -9,5\% (+/- 58.1; -71,6-125,0\%) nach zwölf Monaten. Das durchschnittliche femorale Tunnelenlargement war nach drei Monaten $71,2 \%$ $(+/-56.9 ; 2,2-180,6 \%)$, nach sechs Monaten 62,9\% (+/- 41.5; 11, 1-140,3\%) und nach zwölf Monaten $47,4 \%(+/-43 \cdot 7 ;-36,0-125 \%)$.

Nach zwölf Monaten kam es zu einer Abnahme des durchschnittlichen tibialen Tunnelenlargements im Vergleich zu den Werten drei und sechs Monate postoperativ. Das durchschnittliche tibiale Tunnelenlargement war dabei nach zwölf Monaten signifikant geringer als das tibiale Tunnelenlargement nach drei und sechs Monaten $(p<0,05)$.

Während das durchschnittliche tibiale Tunnelenlargement zwölf Monate postoperativ bei - 9,5\% lag, betrug das durchschnittliche femorale Tunnelenlargement zum selben Zeitpunkt 47,4\%. Nach zwölf Monaten war das durchschnittliche tibiale Tunnelenlargement signifikant geringer im Vergleich zum femoralen Tunnelenlargement $(p<0,05)$.

Bei gesonderter Betrachtung des jeweils gelenknahen Drittels des Bohrkanalabschnittes lag das durchschnittliche tibiale Tunnelenlargement nach drei Monaten bei 63,2\% (+l- 68,9;-21,2-242,2\%) nach sechs Monaten bei $87,7 \%$ (+/- 56,0; 21,9-215, $1 \%$ ) und nach zwölf Monaten bei 85,2 \% (+/-72.6; - 33, 9-233,1\%). Für den gelenknahen Abschnitt des femoralen Bohrkanals betrug das durchschnittliche Tunnelenlargement nach drei Monaten $91,6 \%(+/-55,2 ; 62,5-215,0)$, nach sechs Monaten 92,75 (+/- 36,7; 50,1-135,1\%) und nach zwölf Monaten 78,7\% (+/-50.9; 10,6-164,1\%).

Während die Betrachtung des tibialenTunnelenlargements bezogen auf alle drei Bohrkanalabschnitte zu einer signifikanten Abnahme nach zwölf Monaten führte, kam es bei gesonderter Betrachtung des gelenknahen Bohrkanalabschnittes zu keiner signifikanten Abnahme des tibialen Tunnelenlargements $(p>0,05)$. Ebenfalls kam es bei gesonderter Betrachtung des gelenknahen femoralen Bohrkanalabschnittes zu keiner signifikanten Abnahme des Tunnelenlargements im postoperativen Verlauf $(p>0,05)$.

Eine graphische Übersicht des tibialen und femoralen Tunnelenlargements im zeitlichen Verlauf zeigt Abbilgung 21. 


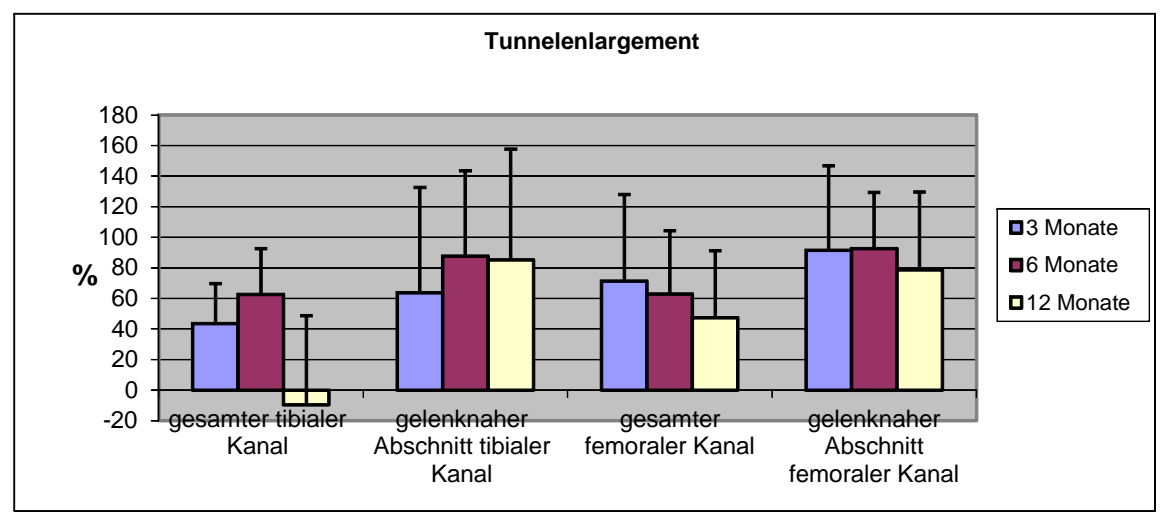

Abbildung 21: Tibiales und femorales Tunnelenlargement nach drei, sechs und zwölf Monaten 


\subsubsection{Tunnelenlargement des tibialen Bohrkanals}

Die Abbildungen 22 a-c zeigen den Verlauf des tibialen Tunnelenlargements bei einem 32 jährigen Patienten drei, sechs und zwölf Monate postoperativ.

Die Abbildungen zeigen, dass im gelenknahen Abschnitt des Bohrkanals das Tunnelenlargement während des Untersuchungszeitraumes am größten war und sich im postoperativen Verlauf nicht reduzierte (Abbildung 22 a-c).
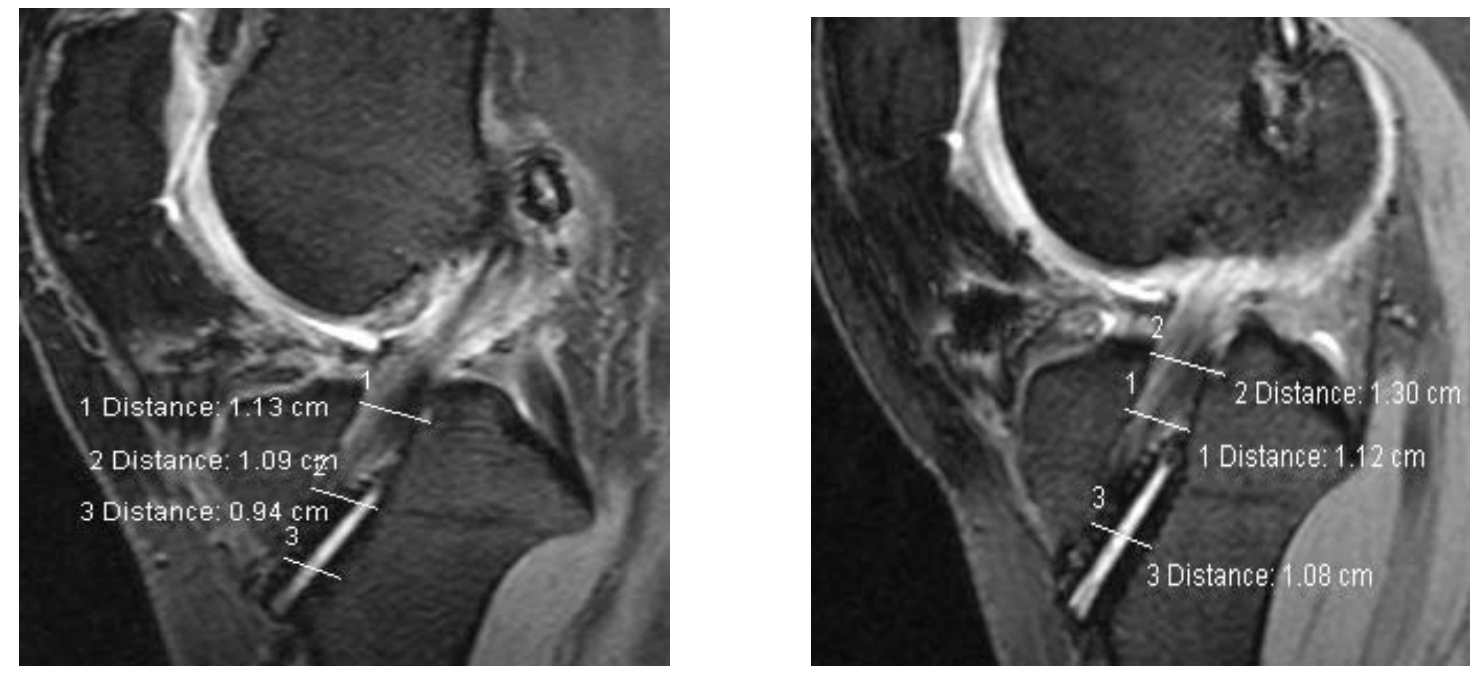

Abbildung 22 a/b: Tibiales Tunnelenlargement drei (a) und sechs (b) Monate nach VKB-Ersatz. Dargestellt ist der maximale Bohrkanaldurchmesser (Tunnelenlargement) für den gelenknahen, den mittleren und den gelenkfernen Bohrkanalabschnitt. Nach sechs Monaten ist die Milagro ${ }^{\circledR}$-Interferenzschraube nahezu vollständig erhalten. Der tibiale Bohrkanal wurde auf gmm aufgebohrt. Der maximale Durchmesser des Bohrkanals liegt im gelenknahen Bohrkanalabschnitt und beträgt nach drei Monaten $1.13 \mathrm{~cm}$ und nach sechs Monaten $1.30 \mathrm{~cm}$. Die prozentuale Zunahme des Bohrkanals im gelenknahen Abschnitt beträgt demnach nach drei Monaten 1,13 $\mathrm{cm} / 0,9 \mathrm{~cm} \times 100 \%=25,6 \%$ und nach sechs Monaten $44,4 \%$.

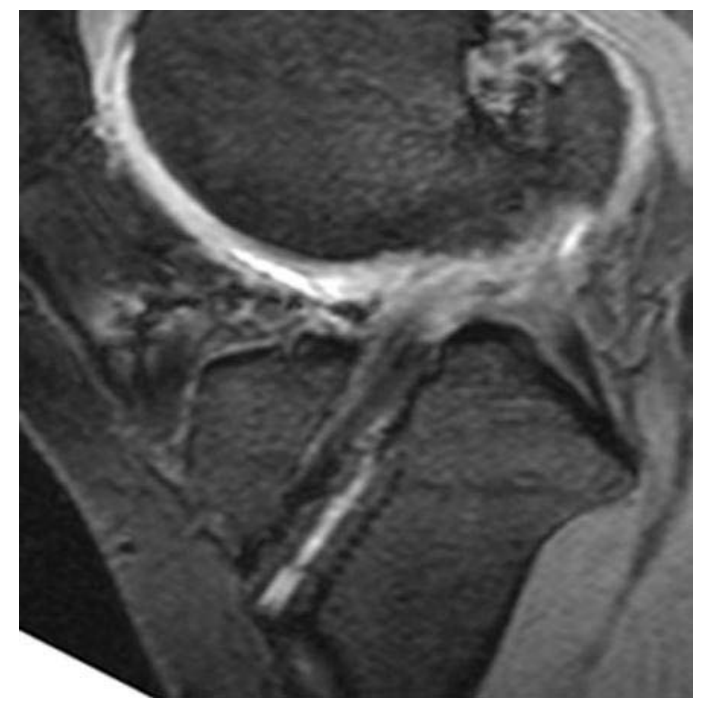

Abbildung 22c:

Tibiales Tunnelenlargement zwölf Monate nach VKB-

Ersatz. Es sind nur noch Reste der Milagro ${ }^{\circledR}$ -

Interferenzschraube zu erkennen. Durch die sich resorbierende Milagro ${ }^{\circledR}$-Interferenzschraube und einsprossendes Knochengewebe nahm das Tunnelenlargement im mittleren und gelenkfernen Bohrkanalabschnitt nach zwölf Monaten ab. Infolgedessen reduzierte sich das tibiale Tunnelenlargement des gesamten Bohrkanals. Das Tunnelenlargement des gelenknahen Bohrkanalabschnittes reduzierte sich nicht. 


\subsubsection{Tunnelenlargement des femoralen Bohrkanals}

Die Abbildungen 23 a-c zeigen den femoralen Bohrkanal eines Patienten in der Längsachse des

Bohrkanals.
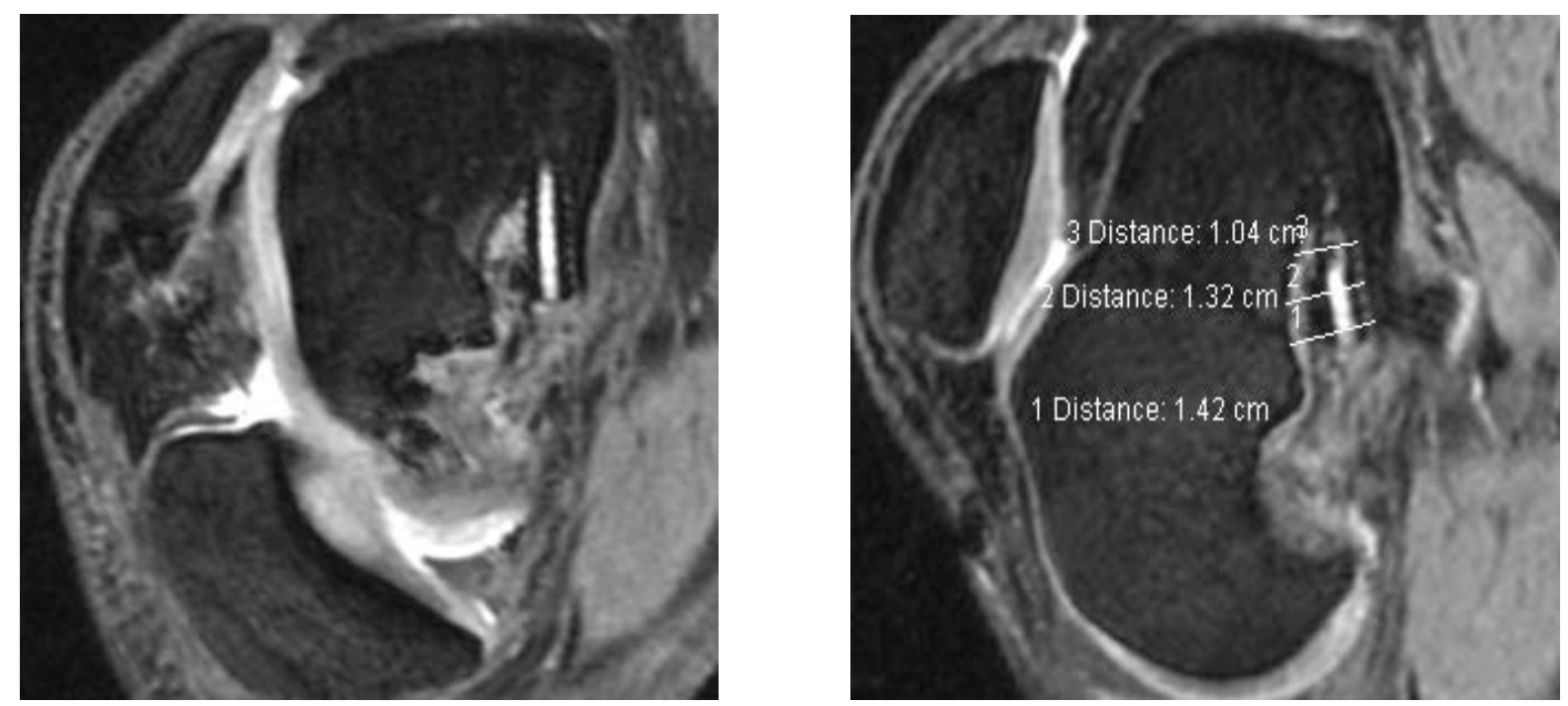

Abbildung 23 a und 23 b aus Frosch et al. (2009, S.77): Femorales Tunnelenlargement drei (a) und sechs (b) Monate nach VKB-Ersatz (mit freundlicher Genehmigung des Springer Verlages).

Der femorale Bohrkanal wurde auf 8mm aufgebohrt. Ein Tunnelenlargement findet sich bereits drei Monate postoperativ und ist im gelenknahen Abschnitt am größten. Nach sechs Monaten zeigt sich eine beginnende Resorption der Milagro ${ }^{\circledR}$-Interferenzschraube.

Abbildung 23 c zeigt das femorale Tunnelenlargement bei diesem Patienten nach zwölf Monaten.

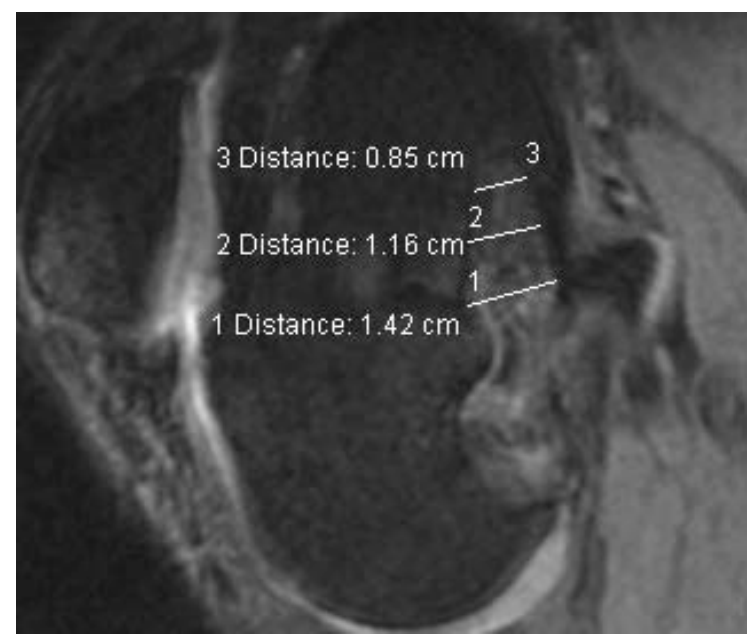

Abbildung 23 c aus Frosch et al. (2009, S.77): Femorales Tunnelenlargement zwölf Monate nach VKB-Ersatz (mit freundlicher Genehmigung des Springer Verlages).

Nach zwölf Monaten ist eine Abnahme des femoralen Tunnelenlargements im mittleren und gelenkfernen Bohrkanalabschnitt im Vergleich zu den Abbildungen 25 a/b erkennbar. Im gelenknahen Bohrkanalabschnitt ist das Tunnelenlargement im postoperativen Verlauf unverändert. Hier kommt es zu keiner Reduktion des maximalen Bohrkanaldurchmessers. Die Milagro ${ }^{\circledR}$-Interferenzschraube ist nach zwölf Monaten vollständig resorbiert. Durch Einwachsen von Knochengewebe in die sich resorbierende Schraube reduziert sich das Tunnelenlargement im gelenkfernen und mittleren Bohrkanalabschnitt. 


\section{8 Ödeme, Knochenzysten und Osteolysen}

Bei einer Patientin fand sich eine Zyste am tibialen Transplantatansatz ohne nachweisbare Fremdkörperreaktion um die Schraube. Der tibiale Bohrkanal war auf 0,85 cm aufgebohrt und vergrößerte sich durch die Osteolyse bereits nach drei Monaten auf $1,48 \mathrm{~cm}$.

Während des weiteren Verlaufs kam es zu keiner Zunahme der Osteolyse. Die tibiale Interferenzschraube war 0,3 cm distal des tibialen Gelenkplateaus platziert. Bei keinem weiteren Untersuchungsteilnehmer konnte während des Untersuchungszeitraums eine Osteolyse oder eine Zyste am tibialen oder femoralen Bohrkanal gefunden werden.

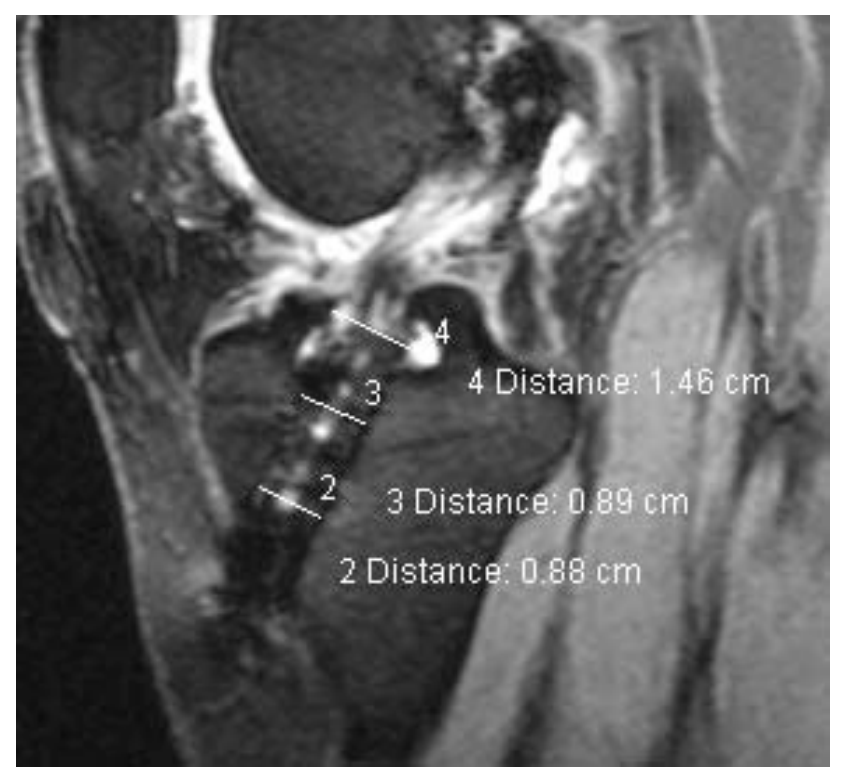

Abbildung 24: Zyste am gelenknahen Abschnitt des tibialen Bohrkanals zwölf Monate nach Ersatz des VKB 


\section{Diskussion}

\subsection{Resorption der Milagro ${ }^{\circledR}$-Interferenzschraube}

Für die Transplantatfixation beim vorderen Kreuzbandersatz kommen häufig Interferenzschrauben zur Anwendung. Herkömmliche bioabsorbierbare Interferenzschrauben bestehen aus resorbierbaren Polymeren. Das Abbauverhalten der verwendeten bioresorbierbaren Materialien ist dabei höchst unterschiedlich (Weiler et. al 2000).

Neben der Zusammensetzung der bioresorbierbaren Materialien scheint die Transplantatwahl das Abbauverhalten der resorbierbaren Polymere zu beeinflussen. Bei der Kreuzbandplastik unter Verwendung der Patellarsehne wird das mittlere Drittel der Patellarsehne mitsamt Knochenzylindern (Bone-Tendon-Bone) aus Patella und Tuberositas tibiae entnommen.

Fink et al. konnten in einer CT-Studie nachweisen, dass Interferenzschrauben aus PGA (polyglycolid acid) bei einer VKB-Ersatzplastik aus einem BTB-Transplantat nach zwölf Monaten vollständig resorbiert werden (Fink et al. 2000). Lajtai et al. zeigten in einer MRT-Studie, dass Interferenzschrauben aus PGA/PLA bei gleicher Transplantatwahl nach sechs Monaten vollständig abgebaut werden (Lajtai et al. 1998). Die mittlere Volumenabnahme von Poly- L lactid acid (PLLA) Interferenzschrauben ohne Glycolid betrug nach zwei Jahren 60\% bei VKB-Ersatz mit einem BTB-Transplantat (Drogset et al. 2006).

Bei einer Kreuzbandplastik aus Hamstringsehnen scheint die Resorption der Interferenzschrauben langsamer als bei einer Ersatzplastik mittels BTB-Transplantat zu sein. Denn bei PLLA Interferenzschrauben, die zur Fixation von Hamstringsehnen benutzt wurden, fand sich auch nach vier Jahren noch keine Resorption (Radford et al. 2005). Ebenfalls konnten Ma et al. in einer MRT-Studie bei PLLA-Schrauben zwei bis vier Jahre nach VKB-Plastik mittels Hamstringsehnen bei keiner Interferenzschraube eine vollständige Resorption nachweisen (Ma et al 2004).

Bekannt ist jedoch auch, dass PLLA im Vergleich zu anderen bioabsorbierbaren Materialien, zum Beispiel Poly (lactid - Co -) Glycolid, langsam über viele Jahre absorbiert wird (Athanasiou et al. 1998). In den direkt postoperativ durchgeführten kernspintomographischen Kontrollen zeigt sich in vorliegender Studie durch den Vergleich von tatsächlichem Schraubendurchmesser und gemessenem Schraubendurchmesser eine Messgenauigkeit von 98\%, sodass gerade in den ersten sechs Monaten von einer hohen Validität der Ergebnisse ausgegangen werden kann. Nach zwölf Monaten, als nur noch Reste der Schrauben zu finden waren, gestalteten sich die Messungen deutlich schwieriger mit sicherlich reduzierter Messgenauigkeit.

Die Bioabsorption der tibialen Milagro ${ }^{\circledR}$-Interferenzschrauben betrug in vorliegender Studie nach sechs Monaten 8,1 \% und nach zwölf Monaten schon 82,57\%. Femoral lag nach sechs Monaten der 
durchschnittliche Volumenverlust bei $31,26 \%$, nach zwölf Monaten ergab sich femoral ein durchschnittlicher Volumenverlust von 92,02\%.

Zu beachten ist allerdings, dass histologische Untersuchungen von den kernspintomographischen Ergebnissen abweichen können. So fanden Morgan et al. heraus, dass das Molekulargewicht einer Schraube, die kernspintomographisch noch deutlich abgegrenzt werden kann, in histologischen Untersuchungen bereits um 75\% abgenommen hat. Die tatsächliche Resorption von bioresorbierbaren Materialien kann den kernspintomographisch ermittelnden Ergebnissen vorausgehen (Morgan et al. 2002). Dies sollte bei der Interpretation der Ergebnisse berücksichtigt werden.

Die Milagro ${ }^{\circledR}$-Schraube besteht neben ß-TCP aus Poly(lactid- Co-) Glycolid. Auch unsere Studienergebnisse bestätigen, dass die Volumenabnahme der Milagro ${ }^{\circledR}$-Schraube schneller voranschreitet als bei bioresorbierbaren Materialien ohne Glycolid. In einer CT evaluierten Studie von Tecklenburg et al. war eine Interferenzschraube, die nur aus PLLA und ß-TCP bestand im Gegensatz zu vorliegenden Ergebnissen nach zwei Jahren nur teilweise abgebaut (Tecklenburg et. al 2006). Polyglycoide scheinen jedoch einen höheren Anteil an Osteolysen zu induzieren als Polymere ohne Glycoide (Kwak et al. 2008). Tecklenburg et. al konnten zwei Jahre nach VKB-Ersatz bei Interferenzschrauben, die aus 75\% PLLA und 25\% $\beta$-TCP bestehen und nicht mit Polyglycoiden angereichert waren, keine Osteolysen finden (Tecklenburg et al. 2006). Drogset et al. konnten jedoch bei Verwendung von PLLA-Schrauben ohne Polyglycoide nach zwei Jahren bei 15,8\% der Patienten im femoralen Bohrkanal Osteolysen nachweisen (Drogset et al.2006).

In vorliegender Studie fand sich bei einer Patientin (5\%) extraartikulär am tibialen Bohrkanal eine Zyste. Bei keinen weiteren Patienten fanden sich während des Untersuchungszeitraumes Osteolysen bei Verwendung von Milagro ${ }^{\circledR}$-Schraube, die mit Glycoiden angereichert sind.

Tibiale extraartikuläre Zysten stellen ein seltenes Phänomen nach VKB-Ersatz dar und wurden sowohl bei Verwendung von PLLA-Schrauben (Martinek und Friederich 1999, Busfield und Anderson 2007), als auch bei Verwendung von PLLA-Schrauben mit osteokonduktiven Substanzen (Dujardin et al. 2008) beobachtet.

Die Osteolyserate der Milagro ${ }^{\circledR}$-Schraube, die aus ß-TCP und Polyglycoide besteht, ist mit $5 \%$ gering. Bei herkömmlichen Biointerferenzschrauben, die meist aus resorbierbaren Polymeren bestehen, konnte bisher ein Ersatz der Schrauben durch Knochengewebe nicht (Tecklenburg et al. 2006, Bach et al. 2002, Barber et al. 2006) oder nur vereinzelt und im Langzeitverlauf beobachtet werden (Laitai et al. 2001).

Durch Anreicherung der Milagro ${ }^{\circledR}$-Schraube mit 30\% ß-TCP soll das Einwachsen von Knochengewebe induziert werden. Bekannt ist dass ß-TCP osteoconductiv wirkt und als Knochenersatzmaterial vollständig durch Knochengewebe ersetzt wird (Bodde et al. 2007). 
In einer CT-Studie von Barber et al. wurden Schrauben, die aus ß-TCP und PLLA bestehen, nach Fixation eines BTB-Transplantates untersucht. Barber et al konnten dabei nachweisen, dass diese Schrauben osteoconduktiv sind und teilweise oder vollständig durch Knochengewebe ersetzt werden (Barber und Dockery 2008).

In vorliegender Studie wurde die Evaluation des Abbauverhaltens kernspintomographisch durchgeführt. Die Osteointegration in vorliegender Studie wurde nicht durch histologische Verfahren bestätigt. Jedoch ist bekannt, dass eine hohe Korrelation zwischen histologischem und kernspintomographischem Befund zu erwarten ist (Hunt et al. 2006).

Auch haben vorklinische Tierstudien gezeigt, dass eine kernspintomographisch angenommene Osteointegration histologisch bestätigt werden konnte (Balch et al. 1999). Die vorliegenden Studienergebnisse scheinen daher zu bestätigen, dass Interferenzschrauben, die ß-TCP enthalten, in vivo osteokonduktiv sind.

Des Weiteren zeichnen sich die Milagro ${ }^{\circledR}$-Schrauben durch ein, der Einheilung des Transplantates angepasstes Resorptionsverhalten aus, da sie in den ersten sechs Monaten nach VKB-Plastik ihre Form weitgehend stabil halten. Nach sechs Monaten werden die Schrauben dann zunehmend resorbiert, so dass nach zwölf Monaten nur noch Schraubenreste nachweisbar sind.

In Tiermodellen konnte gezeigt werden, dass bei Verwendung von Hamstringsehnen und Fixation mit Poly-(D-, L-lactid) Schrauben innerhalb der ersten zwölf Wochen eine direkte Verbindung der Sehnen an der knöchernen Tunnelwand erfolgt (Hunt et al. 2006).

Bis zur vollen Entwicklung der direkten Sehnen-Knochen-Verbindung dauert es bis zu 24 Wochen (Hunt et al. 2006).Gerade innerhalb der ersten drei Monate, wenn für eine sichere Einheilung des Transplantates in den Knochen eine stabile Verankerung gebraucht wird (Logan et al. 2003), bleibt die Milagro ${ }^{\circledR}$-Schraube stabil und resorbiert sich kaum. Auch nach sechs Monaten konnte in vorliegender Studie tibial nur eine geringe Resorption der Milagro ${ }^{\circledR}$-Schrauben nachgewiesen werden.

Erst zwischen sechs und zwölf Monaten, wenn das Transplantat in der Regel eingeheilt ist, kommt es zu einem schnellen Abbau der Schrauben, so dass nach zwölf Monaten durchschnittlich mehr als 9o\% des Schraubenvolumens resorbiert sind. Im Vergleich bleiben die tibialen Milagro ${ }^{\circledR}$-Schrauben nach sechs Monaten signifikant länger erhalten als die femoralen. Auch dies ist wünschenswert, da bei pull-out Tests das Transplantatversagen fast ausschließlich tibial statt fand (Cain et al. 2005).

\subsection{Tunnelenlargement bei Fixation mit der Milagro ${ }^{\circledR}$-Interferenzschraube}

Die genaue Ursache des Tunnelenlargements ist unklar (Höher et al. 1998). Die Ursachen können mechanisch, biologisch oder multifaktoriel sein (Hantes et al. 2004, Wilson et al. 2004). Verschiedene Studien konnten jedoch zeigen, dass das Ausmaß des Tunnelenlargements keine Auswirkungen auf das klinische Ergebnis hat (Clathworthy et al. 1999, Buelow et al. 2002). Nichts desto 
trotz kann ein ausgeprägtes Tunnelenlargement zu erheblichen Problemen beim chirurgischen Revisionseingriff führen (Getelman und Friedman 1999). Deshalb ist es wichtig, mögliche Faktoren, die beim chirurgischen Primäreingriff zu einem Tunnelenlargement führen, zu vermeiden.

Zu beachten ist, dass die Messung des Tunnelenlargements in der Literatur in unterschiedlicher Art und Weise erfolgt. Buelow et al. bestimmten das tibiale Tunnelenlargement im konventionell radiologischen Röntgenbild $1 \mathrm{~cm}$ distal des Ansatzes des VKB. Analog erfolgte die Messung für das femorale Tunnelenlargement $1 \mathrm{~cm}$ proximal des Ansatzes des VKB (Buelow et al. 2002). Ma et al. berechneten das Tunnelenlargement an drei Messpunkten für jeden Bohrkanal, wobei die Messpunkte jeweils $5 \mathrm{~mm}$ voneinander entfernt lagen (Ma et al. 2004).

In vorliegender Studie wurde das Tunnelenlargement nicht nur für den gelenknah gelegenen, sondern auch für den mittleren und gelenkfernen Bohrkanalabschnitt gemessen. Um vorliegende Studienergebnisse mit denen der Literatur besser vergleichen zu können, wurden die Ergebnisse des gelenknahen Tunnelenlargements gesondert ausgewertet.

Bei Betrachtung der Ergebnisse zeigt sich, dass das Tunnelenlargement des gesamten Bohrkanals während des Nachuntersuchungszeitraumes abnimmt und deutlich niedriger ist, als das Tunnelenlargement des gelenknahen Bohrkanalabschnitts. Besonders im gelenkfernen tibialen Bohrkanalabschnitt kam es zwölf Monate nach VKB-Ersatz zur deutlichen Reduktion des Tunnelenlargements, da die sich resorbierende Milagro ${ }^{\circledR}$-Schraube teilweise durch Knochengewebe ersetzt wurde.

Die vorliegende Studie zeigt, dass durch die Verwendung von Milagro ${ }^{\circledR}$-Schrauben das Tunnelenlargement in den ersten sechs Monaten nach VKB-Ersatz nicht vermieden werden kann. Im weiteren Verlauf kommt es jedoch durch induzierte Ossifikation zu einer signifikanten Reduktion des Tunnelenlargements des gesamten Bohrkanals. Trotz ß-TCP bedingter induzierter Osteointegration kann das Tunnelenlargement für die gelenknahen Bohrkanalabschnitte nicht vermieden werden. Jedoch kann, durch den kernspintomographisch angenommenen Ersatz der Schrauben durch Knochengewebe, das Gesamtvolumen des Bohrkanals reduziert werden. Nach zwölf Monaten war der durchschnittliche tibiale Bohrkanaldurchmesser durch einsprossendes Knochengewebe signifikant geringer im Vergleich zu den Werten nach drei und sechs Monaten. Die Ossifikation des Bohrkanals hat eine Reduktion des Bohrkanalvolumens zur Folge.

In vorliegender Studie betrug das Tunnelenlargement des gelenknahen tibialen Bohrkanalabschnittes nach zwölf Monaten 85,2 \%. Femoral betrug die prozentuale Zunahme für den gelenknahen Bohrkanalabschnitt nach zwölf Monaten 78,7\%. Damit ergeben sich für die gelenknahen Bohrkanalabschnitte ähnliche Werte wie sie in der Literatur zu finden sind. Nach sechs Monaten betrug bei Clathworthy et al. das femorale Tunnelenlargement 100, $4 \%$ und das tibiale Tunnelenlargement $73,9 \%$ (Clathworthy et al. 1999). 
Buelow et al. berechneten bereits zwei Tage postoperativ ein Tunnelenlargement von tibial $73 \%$ und femoral 78\%. Buelow et al. sind daher der Meinung, dass durch das Eindrehen der Interferenzschrauben nicht nur das Transplantat an die Bohrkanalwand fixiert wird, sondern das Eindrehen selbst ein Tunnelenlargement verursacht. Nach sechs Monaten berechneten Buelow et al. ein tibiales Tunnelenlargement von 94\% und ein femorales Tunnelenlargement von 119\%. Im weiteren Verlauf änderte sich das Tunnelenlargement kaum noch (Buelow et al. 2002).

Auch bei den hier vorliegenden Studienergebnissen ist die prozentuale Zunahme des gelenknahen Bohrkanaldurchmessers in den ersten drei Monaten am größten.

Ein weiterer Faktor, der erklärt, weshalb mit zunehmender Untersuchungsdauer das gelenknahe Tunnelenlargement weniger stark zunimmt, ist die Einheilung des Transplantates in die Bohrkanalwand. Denn bis zur vollen Entwicklung einer direkten Transplantat-Knochen-Verbindung vergehen bis zu zwölf Wochen (Yoshiya et al. 2000). Nachdem sich eine direkte Verbindung zwischen Transplantat und Bohrkanalwand nach drei Monaten ausgebildet hat, werden Mikrobewegungen des Transplantates im Bohrkanal vermieden. Dies kann eine weitere Zunahme des Tunnelenlargements verhindern (Wilson et al. 2004).

Die Mobilität des Transplantates im Bohrkanal wird in vielen Studien, als der dominierende Faktor für das Tunnelenlargement gesehen. L' Insalata et al. gehen davon aus, dass gelenkferne Fixationstechniken das Eindringen von Synovialflüssigkeit in den Bohrkanal ermöglicht, was eine vermehrte Mobilität des Transplantates in der Sagittalebene zur Folge hat. Um diese, von L'Insalata et al. auch als „winshield wiper effect" genannten, Bewegungen und damit ein Tunnelenlargement zu vermeiden, wird eine gelenknahe Transplantatfixation empfohlen (L'Insalata et al. 1998, S.235). Ebenso kann es durch den „bungee effect" bei gelenkfernen Fixationstechniken zur Längenänderung des Transplantates und infolgedessen zum verzögerten Einheilen des Transplantates mit resultierendem Tunnelenlargement kommen (Höher et al. 1998, S.237; Uchio et al. 2002).

Klinische Ergebnisse bei Transplantatfixation mit Milagro ${ }^{\circledR}$ Interferenzschrauben

Die vorliegende Studie konnte zeigen, dass bei Transplantatfixation mit Milagro ${ }^{\circledR}$-Interferenzschrauben gute klinische Ergebnisse erreicht werden. Im Marshall Score konnte für die Gruppe "Semitendinosus" ein signifikant besseres Ergebnis als für die Gruppe „Semitendinosus und Gracilis" nachgewiesen werden. Für den Tegner Score, den Lysholm Score, den IKDC-Score und die VAS Schmerz und Funktion gab es keine signifikanten Unterschiede zwischen den Untersuchungsgruppen. 
Gobbi et al. konnten bereits zeigen, dass es keine signifikanten Unterschiede im klinischen Outcome gibt, wenn die Rekonstruktion des VKB ohne die Gracilissehne erfolgt. Im Unterschied zur vorliegenden Studie erfolgte hierbei die femorale Transplantatfixation mit einem EndoButton.

Die Mm. Semitendinosus und Gracilis sind sowohl Innenrotatoren der Tibia als auch Flexoren im Kniegelenk. Gobbi et al. fanden in isokinetischen Krafttests heraus, dass bei Entnahme der Gracilis- und Semitendinosussehne das maximale Drehmoment bei Innenrotation der Tibia signifikant niedriger ist, als bei alleiniger Entnahme der Semitendinosussehne (Gobbi et al. 2005).

Tashiro et al. wiesen zudem nach, dass eine zusätzliche Entnahme der Gracilissehne eine Kraftminderung im Oberschenkelbeugebereich zur Folge hat. Bekannt ist des Weiteren, dass ein Jahr nach Entnahme beider Sehnen zur VKB-Rekonstruktion ein Defizit der Hamstringmuskulatur besteht (Burks et al. 2005).

Wie in vorliegender Studie fanden sich auch bei Tashiro et al. keine signifikanten Unterschiede im klinischen Ergebnis bei Vergleich einer Gruppe "Semitendinosus" und einer Gruppe „Semitendinosus und Gracilis". Im Unterschied zur vorliegenden Studie erfolgte auch in der Studie von Tashiro et al. die Transplantatfixation mittels EndoButton (Tashiro et al. 2003). Tashiro et al. sowie Gobbi et al. empfehlen daher, falls möglich auf die Gracilissehne bei einer VKB-Rekonstruktion zu verzichten. Die vorliegenden Studienergebnisse zeigen ebenfalls, dass ein suffizienter VKB-Ersatz aus alleiniger Semitendinosussehne und mittels Transplantatfixation durch Milagro ${ }^{\circledR}$-Interferenzschrauben erreicht wird.

Allerdings ist auch bekannt, dass sich die Hamstringsehnen nach Entnahme regenerieren können und es zu keiner wesentlichen Atrophie kommt (Papandrea et al. 2000, Eriksson et al. 1999).

Darüber hinaus zeigen unsere Studienergebnisse, dass die ventrale Translation des Kniegelenkes im KT 1000 Arthrometer bei einer Zugkraft von $67 \mathrm{~N}$ und beim Manual maximal Drawer (MMD) in der Gruppe "Semitendinosus" tendenziell größer als in der Gruppe „Semitendinosus und Gracilis" ist. Signifikante Unterschiede zwischen den Untersuchungsgruppen ergaben sich bei der Messung mit dem KT 1000 Arthrometer jedoch nicht. Unklar ist, ob eine VKB-Ersatzplastik aus alleiniger Semitendinosussehne eine vermehrte ventrale Translation des Kniegelenks zur Folge haben kann. Studienergebnisse, die dies beantworten finden sich derzeit nicht.

Um eine zusätzliche Schwächung der Innenrotatoren der Tibia sowie der Beuger im Kniegelenk zu vermeiden, sollte daher bei VKB-Rekonstruktion auf die Gracilissehne als Transplantat wenn immer möglich verzichtet werden.

Weitere Studien sollten durchgeführt werden, die untersuchen, inwiefern eine VKB-Plastik aus alleiniger Semitendinosussehne eine vermehrte ventrale Translation im Kniegelenk zur Folge hat. 


\subsection{Schlussfolgerung}

Vorteile der Milagro ${ }^{\circledR}$-Schrauben sind die Darstellbarkeit im konventionellen Röntgen, ein adaptiertes Resorptionsverhalten mit hoher Stabilität in den ersten sechs Monaten und nahezu vollständiger Resorption nach zwölf Monaten mit dem Einwachsen von Knochengewebe in die Schrauben. Die klinischen Ergebnisse bei Transplantatfixation mit Milagro ${ }^{\circledR}$-Interferenzschrauben sind gut und mit anderen Studienergebnissen vergleichbar. Die Osteolyserate war trotz der schnellen Resorption der Milagro ${ }^{\circledR}$-Interferenzschrauben mit $5 \%$ gering.

Die Zunahme des Tunnelenlargements war in den ersten drei Monaten nach der Operation am größten. Durch Einwachsen von Knochengewebe in die sich resorbierende Milagro ${ }^{\circledR}$-Schraube kam es nach zwölf Monaten zur signifikanten Reduktion des Bohrkanalvolumens. Dies kann vor allem bei einem möglichen Revisionseingriff von Bedeutung sein.

Bei gesonderter Betrachtung des gelenknahen Bohrkanalabschnittes konnte das Tunnelenlargement in vorliegender Studie im Vergleich zu Vorstudien nicht reduziert werden.

In der vorliegenden Arbeit fand sich kein signifikanter Unterschied zwischen der Verwendung der Semitendinosussehne alleine und einer Kombination aus Semitendinosus- und Gracilissehne als Kreuzbandersatztransplantat.

Das klinische Ergebnis in den visuellen Analogskalen zu Schmerz und Funktion, in Tegner Score, Lysholm Score sowie bei der Stabilitätsprüfung mit dem KT 1000 Arthrometer ist für beide Untersuchungsgruppen vergleichbar. Lediglich für den Marshall Score zeigte sich bei Verwendung von alleiniger Semitendinosussehne ein signifikant besseres Ergebnis. 


\section{Zusammenfassung}

Die Transplantatfixation mit bioresorbierbaren Interferenzschrauben gehört zu den Standardverfahren beim vorderen Kreuzbandersatz. Ziel vorliegender Studie ist es, das klinische Ergebnis, das Abbauverhalten und die Biokompatibilität der neuartigen Interferenzschraube Milagro ${ }^{\circledR}$ (Mitek, Norderstedt, Deutschland) in vivo zu untersuchen. Die Milagro ${ }^{\circledR}$-Interferenzschraube enthält osteokonduktive Substanzen, die die knöcherne Regeneration des Bohrkanals unterstützen sollen.

In unserer Klinik erfolgte von $06 / 2005$ bis $10 / 2006$ bei 35 Patienten die Transplantatfixation mittels Milagro ${ }^{\circledR}$-Schrauben in arthroskopischer Technik. Bei 15 Patienten wurde als Transplantat die Semitendinosussehne alleine und bei 20 Patienten die Semitendinosus- in Kombination mit der Gracilissehne verwendet. Aus diesen 35 Patienten wurden zufällig zwölf Patienten ausgewählt und nach drei, sechs und zwölf Monaten eine MRT-Untersuchung durchgeführt. Dabei wurde der Volumenverlust der Schrauben und das Tunnelenlargement ermittelt, nach Osteolysen gesucht und evaluiert, ob es im Verlauf zur knöchernen Regeneration des Bohrkanals kam. Bei allen 35 Patienten wurde nach zwölf Monaten der Tegner-, der Lysholm-, der Marshall-, der IKDC-Score, die visuelle Analogskala Schmerz und Funktion und die ventrale Translation mit dem KT-1000 Arthrometer ermittelt. Es wurde zusätzlich untersucht, ob es Unterschiede im klinischen Ergebnis bei VKB-Rekonstruktion mit der Semitendinosussehne (ST) alleine versus Semitendinosus- und Gracilissehne (STG) als Transplantat gibt.

Beim Gesamtkollektiv lag der mittlere Lysholm Score nach einem Jahr bei 93,4 (+/- 5,4) Punkten, der mittlere Marshall Score bei 46,6 (+/-2,5) Punkten. Die Stabilitätsprüfung mit dem KT-1000 Arthrometer ergab eine durchschnittliche Seitendifferenz von $1,61 \mathrm{~mm}(+/-1,1)$ bei einem Kraftaufwand von $67 \mathrm{~N}$, $1,94 \mathrm{~mm}(+/-1,1)$ bei einem Kraftaufwand von $89 \mathrm{~N}$ und $2,19 \mathrm{~mm}(+/-1,3)$ bei der maximalen manuellen Auslenkung.

Bei Betrachtung des Lysholm Scores, Tegner Scores, der visuellen Analogskala Schmerz und Funktion sowie der Stabilitätsprüfung mit dem KT-1000 Arthrometer fanden sich keine signifikanten Unterschiede bei Patienten mit alleiniger Semitendinosussehne als Transplantat und Patienten mit einer Kombination aus Semitendinosus- und Gracilissehne.

Die Patienten mit alleiniger Semitendinosussehne erreichten im Marshall Score eine durchschnittliche Punktzahl von $47,7(+/-2,1)$. Patienten mit einer Kombination aus Semitendinosus- und Gracilissehne erreichten im Marshall Score eine durchschnittliche Punktzahl von 45,8 (+/- 2,5). Hinsichtlich des Marshall Scores wurde für Patienten, deren Transplantat aus alleiniger Semitendinosussehne bestand, ein signifikant besseres Ergebnis erreicht. 
Bei den tibialen Schrauben war der durchschnittliche Volumenverlust nach sechs und zwölf Monaten 8,1 $(+/-7,9) \%$ und 82,5 (+/-17,2) \%. Bei den femoralen Schrauben kam es nach drei, sechs und zwölf Monaten zu einem Volumenverlust von 2,5 (+/- 2,1) \%, 31,3 (+/- 21,6) \% und 92,0 (+/- 6,0) \%. Der Anteil an Osteolysen war dabei gering und lag bei $5 \%$. Für die gelenknahen Bohrkanalabschnitte nahm nach zwölf Monaten der Bohrkanaldurchmesser um 85,2 (+/- 72,6) \% zu, femoral betrug die Zunahme 78,7 (+/- 50.9) \%.

Die vorliegende Studie zeigt, dass die sich resorbierende Milagro ${ }^{\circledR}$-Schraube teilweise durch Knochengewebe ersetzt wird. Durch die kernspintomographisch nachgewiesene knöcherne Regeneration der sich resorbierenden Milagro ${ }^{\circledR}$-Schraube kam es zur Reduktion des Bohrkanalvolumens. Die Milagro ${ }^{\circledR}$-Schrauben zeichnen sich durch ein der Einheilung des Kreuzbandes angepasstes Resorptionsverhalten aus, da sie in den ersten sechs Monaten nach VKB-Ersatz kaum resorbiert werden, jedoch anschließend eine schnelle Volumenabnahme erfolgt. Ein weiterer Vorteil der Milagro ${ }^{\circledR}$-Schrauben ist, dass diese im konventionellen Röntgenbild sichtbar ist. 


\section{Literaturverzeichnis}

Amis AA, Dawkins GP (1991): Functional anatomy of the anterior cruciate ligament. Fibre bundle actions related to ligament replacements and injuries. J Bone Joint Surg Br 73, 260-267

Athanasiou KA, Agrawal CM, Barber FA, Burkhart SS (1998): Orthopaedic applications for PLA-PGA biodegradable polymers. Arthroscopy 14, 726-737

Bach FD, Carlier RY, Elis JB, Mompoint DM, Feydy A, Judet O et al. (2002): Anterior cruciate ligament reconstruction with bioabsorbable polyglycolic acid interference screws: MR imaging follow-up.

Radiology 225, 541-550

Balch OK, Collier MA, DeBault LE, Johnson LL (1999): Bioabsorbable suture anchor (co-polymer 85/15 $\mathrm{D}, \mathrm{L}$ lactide/glycolide) implanted in bone: correlation of physical/mechanical properties, magnetic resonance imaging, and histological response. Arthroscopy 15, 691-708

Barber FA, Dockery WD (2006): Long-term absorption of poly-L-lactic Acid interference screws. Arthroscopy 22, 820-826

Barber FA, Dockery WD (2008): Long-term absorption of beta-tricalcium phosphate poly-L-lactic acid interference screws. Arthroscopy 24, 441-447

Barber FA, Elrod BF, McGuire DA, Paulos LE (1995): Preliminary results of an absorbable interference screw. Arthroscopy 11, 537-548

Battaglia TC, Miller MD (2005): Management of bony deficiency in revision anterior cruciate ligament reconstruction using allograft bone dowels: surgical technique. Arthroscopy $\underline{21}, 767$

Bodde EWH, Wolke JGC, Kowalski RSZ, Jansen JA (2007): Bone regeneration of porous beta-tricalcium phosphate (Conduit TCP) and of biphasic calcium phosphate ceramic (Biosel) in trabecular defects in sheep. J Biomed Mater Res A $\underline{82}, 711-722$

Böstman O, Hirvensalo E, Mäkinen J, Rokkanen P (1990): Foreign-body reactions to fracture fixation implants of biodegradable synthetic polymers. J Bone Joint Surg Br 72, 592-596

Brown CHJ, Carson EW (1999): Revision anterior cruciate ligament surgery. Clin Sports Med 18, 109-171

Buelow JU, Siebold R, Ellermann A (2002): A prospective evaluation of tunnel enlargement in anterior cruciate ligament reconstruction with hamstrings: extracortical versus anatomical fixation. Knee Surg Sports Traumatol Arthrosc 10, 80-85

Burks RT, Crim J, Fink BP, Boylan DN, Greis PE (2005): The effects of semitendinosus and gracilis harvest in anterior cruciate ligament reconstruction. Arthroscopy 211, 1177-85

Busfield BT, Anderson LJ (2007): Sterile pretibial abscess after anterior cruciate reconstruction from bioabsorbable interference screws: a report of 2 cases. Arthroscopy 23, 911.e1-4

Butler DL, Noyes FR, Grood ES (1980): Ligamentous restraints to anterior-posterior drawer in the human knee. A biomechanical study. J Bone Joint Surg Am 62, 259-270

Cain EL, Phillips BB, Charlebois SZ, Azar FM (2005): Effect of tibial tunnel dilation on pullout strength of semitendinosus-grcilis-graft in anterior cruciate ligament reconstruction. Orthopedics $\underline{28}, 779-783$ 
Cameron M, Fu FH, Paessler HH, Schneider M, Evans CH (1994): Synovial fluid cytokine concentrations as possible prognostic indicators in the ACL-deficient knee. Knee Surg Sports Traumatol Arthrosc 2 , 3844

Cameron M, Buchgraber A, Passler H, Vogt M, Thonar E, Fu F, Evans CH (1997): The natural history of the anterior cruciate ligament-deficient knee. Changes in synovial fluid cytokine and keratan sulfate concentrations. Am J Sports Med 25, 751-754

Clathworthy MG, Annear P, Buelow JU, Bartlett RJ (1999) Tunnel widening in anterior cruciate ligament reconstruction: a prospective evaluation of hamstrings and patella tendon grafts. Knee Surg, Sports Traumatol Arthrosc Z, 138-145

Daniel DM, Stone ML, Sachs R, Malcom L (1985): Instrumented measurement of anterior knee laxity in patients with acute anterior cruciate ligament disruption. Am J Sports Med 13, 401-407

Drogset JO, Grontvedt T, Myhr G (2006): Magnetic resonance imaging analysis of bioabsorbable interference screws used for fixation of bone-patellar tendon-bone autografts in endoscopic reconstruction of the anterior cruciate ligament. Am J Sports Med 34, 1164-1169

Dujardin J, Vandenneucker $\mathrm{H}$, Bellemans J (2008): Tibial cyst and intra-articular granuloma formation after anterior cruciate ligament reconstruction using polylactide carbonate osteoconductive interference screws. Arthroscopy 24, 238-242

Eriksson K, Larsson H, Wredmark T, Hamberg P (1999): Semitendinosus tendon regeneration after harvesting for $\mathrm{ACL}$ reconstruction. A prospective MRI study. Knee Surg Sports Traumatol ArthrosC Z, 220-5

Fink C, Hoser C, Benedetto KP (1993): Sports capacity after rupture of the anterior cruciate ligament-surgical versus non-surgical therapy. Aktuelle Traumatol 23, 371-375

Fink C, Benedetto KP, Hackl W, Hoser C, Freund MC, Rieger M (2000). Bioabsorbable polyglyconate interference screw fixation in anterior cruciate ligament reconstruction: a prospective computed tomography-controlled study. Arthroscopy 16, 491-498

Fischer O: Kinematik organischer Gelenke; Vieweg Verlag, Braunschweig 1907

Friedman RJ, Black J, Galante JO, Jacobs JJ, Skinner HB (1994): Current concepts in orthopaedic biomaterials and implant fixation. Instr Course Lect 43, 233-255

Frosch KH, Habermann F, Fuchs M, Michel A, Junge R, Schmidtmann U, Stürmer KM (2001): Is prolonged ambulatory physical therapy after anterior cruciate ligament-plasty indicated? Comparison of costs and benefits. Unfallchirurg 104, 513-518

Frosch KH, Sawallich T, Schütze G, Losch A, Walde T, Balcarek P, Konietschke F, Stürmer KM (2009): Magnetic resonance imaging analysis of the bioabsorbable Milagro ${ }^{\circledR}$ interference screw for graft fixation in anterior cruciate ligament reconstruction. Strat Traum Limb Recon 4, 73-79

Furman W, Marshall JL, Girgis FG (1976): The anterior cruciate ligament. A functional analysis based on postmortem studies. J Bone Joint Surg Am 58, 179-185

Gabriel MT, Wong EK, Woo SL, Yagi M, Debski RE (2004): Distribution of in situ forces in the anterior cruciate ligament in response to rotatory loads. J Orthop Res $\underline{22}, 85-89$ 
Galway HR, Maclntosh DL (1980): The lateral pivot shift: a symptom and sign of anterior cruciate ligament insufficiency. Clin Orthop Relat Res 147, 45-50

George MS, Dunn WR, Spindler KP (2006): Current concepts review: revision anterior cruciate ligament reconstruction. Am J Sports Med 34, 2026-2037

Getelman MH, Friedman MJ(1999): Revision anterior cruciate ligamentreconstruction surgery. J Am Acad Orthop Surg Z,189-198

Girgis FG, Marshall JL, Monajem A (1975): The cruciate ligaments of the knee joint. Anatomical, functional and experimental analysis. Clin Orthop Relat Res 106, 216-231

Gobbi A, Domzalski M, Pascual J, Zanazzo M (2005): Hamstring anterior cruciate ligament reconstruction: is it necessary to sacrifice the gracilis. Arthroscopy 21, 275-280

Gruber J, Wolter D, Lierse W (1986): Anterior cruciate ligament reflex (LCA reflex). Unfallchirurg $\underline{89}$ $551-554$

Halata Z, Haus J (1989): The ultrastructure of sensory nerve endings in human anterior cruciate ligament. Anat Embryol 179, 415-421

Hantes ME, Mastrokalos DS, Yu J, Paessler HH (2004): The effect of early motion on tibial tunnel widening after anterior cruciate ligament replacement using hamstring tendon grafts. Arthroscopy $\underline{20}$, $572-580$

Hefti F, Müller W (1993): Current state of evaluation of knee ligament lesions. The new IKDC knee evaluation form. Orthopade 22, 351-362

Höher J, Moller HD, Fu FH (1998): Bone tunnel enlargement after anterior cruciate ligament reconstruction: fact or fiction. Knee Surg Sports Traumatol Arthrosc $\underline{6}$, 231-240

Hunt P, Rehm O, Weiler A (2006): Soft tissue graft interference fit fixation: observations on graft insertion site healing and tunnel remodeling 2 years after $A C L$ reconstruction in sheep. Knee Surg Sports Traumatol Arthrosc 14, 1245-1251

Johnson DL, Swenson TM, Irrgang JJ, Fu FH, Harner C D (1996): Revision anterior cruciate ligament surgery: experience from Pittsburgh. Clin Orthop Relat Res 325, 100-109

Krudwig WK (2000): Situation der Arthroskopie in Deutschland. Arthroskopie 13, 191-193

Kubein-Meesenburg D, Nägerl H, Cotta H, Fanghänel J (1993): Biomechanical principles in diarthroses and synarthroses. I: Basic concepts in diarthroses. Z Orthop Ihre Grenzgeb 131, 97-104

Kurzweil PR, Frogameni AD, Jackson DW (1995): Tibial interference screw removal following anterior cruciate ligament reconstruction. Arthroscopy 11, 289-291

Kwak JH, Sim JA, Kim SH, Lee KC, Lee BK (2008): Delayed intra-articular inflammatory reaction due to poly-L-lactide bioabsorbable interference screw used in anterior cruciate ligament reconstruction.

Arthroscopy 24, 243-246 
Lajtai G, Balon R, Humer K, Aitzetmüller G, Unger F, Orthner E (1998): Resorbable interference screws. Histologic study 4.5 years postoperative. Unfallchirurg 101, 866-869

Lajtai G, Schmiedhuber G, Unger F, Aitzetmüller G, Klein M, Noszian I, Orthner E (2001): Bone tunnel remodeling at the site of biodegradable interference screws used for anterior cruciate ligament reconstruction: 5-year follow-up. Arthroscopy 17, 597-602

L'Insalata JC, Klatt B, Fu FH, Harner CD (1998): Tunnel expansion following anterior cruciate ligament reconstruction: a comparison of hamstring and patellar tendon autografts. Knee Surg Sports Traumatol Arthrosc 5, 234-238

Linko, E.; Harilainen, A.; Malmivaara, A.; Seitsalo, S. (2005): Surgical versus conservative interventions for anterior cruciate ligament ruptures in adults. In: Cochrane database of systematic reviews, H. 2, S. CDo01356. Online verfügbar unter doi:10.1002/14651858.CDoo1356.pub3.

Logan M, Williams A, Myers P (2003): Is bone tunnel osseointegration in hamstring tendon autograft anterior cruciate ligament reconstruction important? Arthroscopy 19, E1-3

Lysholm J, Gillquist J (1982): Evaluation of knee ligament surgery results with special emphasis on use of a scoring scale. Am J Sports Med 10, 150-154

Ma CB, Francis K, Towers J, Irrgang J, Fu FH, Harner CH (2004): Hamstring anterior cruciate ligament reconstruction: a comparison of bioabsorbable interference screw and endobutton-post fixation. Arthroscopy 20, $122-128$

Macarini L, Murrone M, Marini S, Mocci A, Ettorre GC (2004): MRI in ACL reconstructive surgery with PDLLA bioabsorbable interference screws: evaluation of degradation and osteointegration processes of bioabsorbable screws. Radiol Med 107, 47-57

Majewski M, Schulte KR, Harner CD (2002): Tibial tunnel enlargement after arthroscopic anterior cruciate ligament reconstruction. Sportverletz Sportschaden 16, 22-25

Markolf KL, Bargar WL, Shoemaker SC, Amstutz HC (1981): The role of joint load in knee stability. J Bone Joint Surg Am $\underline{6}, 570-585$

Marshall JL, Fetto JF, Botero PM (1977): Knee ligament injuries: a standardized evaluation method. Clin Orthop Relat Res 123, 115-129

Martinek V, Friederich NF (1999): Tibial and pretibial cyst formation after anterior cruciate ligament reconstruction with bioabsorbable interference screw fixation. Arthroscopy 15, 317-20

Miller RK, Goodfellow JW, Murray DW , O'Connor JJ (1998): In vitro measurement of patellofemoral force after three types of knee replacement. J Bone Joint Surg Br $\underline{80}$, 900-906

Montjovent MO, Mathieu L, Schmoekel H, Mark S, Bourban PE, Zambelli PY et al. (2007): Repair of critical size defects in the rat cranium using ceramic-reinforced PLA scaffolds obtained by supercritical gas foaming. J Biomed Mater Res A 83, 41-51

Morgan CD, Gehrmann RM, Jayo MJ, Johnson CS (2002): Histologic findings with a bioabsorbable anterior cruciate ligament interference screw explant after 2.5 years in vivo. Arthroscopy 18 , E47 
Nägerl H, Kubein-Meesenburg D, Cotta H, Fanghänel J (1993): [Biomechanical principles of diarthroses and synarthroses. III: Mechanical aspects of the tibiofemoral joint and role of the cruciate ligaments]. Z Orthop Ihre Grenzgeb 131, 385-396

Nägerl H, Frosch KH, Wachowski MM, Dumont C, Abicht C, Adam P, Kubein-Meesenburg D (2008): A novel total knee replacement by rolling articulating surfaces. In vivo functional measurements and tests. Acta Bioeng Biomech 10, 55-60

Pandit H, Ward T, Hollinghurst D, Beard DJ, Gill HS, Thomas NP, Murray DW (2005): Influence of surface geometry and the cam-post mechanism on the kinematics of total knee replacement. J Bone Joint Surg 8 z, 940-945

Papandrea P, Vulpiani MC, Ferretti A, Conteduca F (2000): Regeneration of the semitendinosus tendon harvested for anterior cruciate ligament reconstruction. Evaluation using ultrasonography. Am J Sports Med 28, 556-61

Pinskerova V, Johal P, Nakagawa S, Sosna A, Williams A, Gedroyc W, Freeman MA (2004): Does the femur roll-back with flexion? J Bone Joint Surg $\mathrm{Br} \underline{86}$, 925-31

Radford MJ, Noakes J, Read J, Wood DG (2005) : The natural history of a bioabsorbable interference screw used for anterior cruciate ligament reconstruction with a 4-strand hamstring technique.

Arthroscopy 21, 707-710

Schmalzried TP, Akizuki KH, Fedenko AN, Mirra J (1997): The role of access of joint fluid to bone in periarticular osteolysis. A report of four cases. J Bone Joint Surg Am 79, 447-452

Schultz RA, Miller DC, Kerr CS, Micheli L (1984): Mechanoreceptors in human cruciate ligaments. A histological study. J Bone Joint Surg Am $\underline{66}$, 1072-1076

Shelbourne KD, Nitz P (1990): Accelerated rehabilitation after anterior cruciate ligament reconstruction. Am J Sports Med 18, 292-299

Strobel MJ, Stedtfeld HW: Diagnostik des verletzten Kniegelenkes, 1. Auflage; Marseille Verlag, München 1988

Tashiro T, Kurosawa H, Kawakami A, Hikita A, Fukui N (2003): Influence of Medial Hamstring Tendon Harvest on Knee Flexor Strength after Anterior Cruciate Ligament Reconstruction: A Detailed Evaluation with Comparison of Single- and Double-Tendon Harvest. Am J Sports Med 31, 522-529

Tecklenburg K, Burkart P, Hoser C, Rieger M, Fink C (2006): Prospective evaluation of patellar tendon graft fixation in anterior cruciate ligament reconstruction comparing composite bioabsorbable and allograft interference screws. Arthroscopy 22, 993-999

Tegner Y, Lysholm J (1985): Rating systems in the evaluation of knee ligament injuries. Clin Orthop Relat Res $198,43-49$

Uchio Y, Ochi M, Sumen Y, Adachi N, Kawasaki K, Iwasa J, Katsube K (2002): Mechanical properties of newly developed loop ligament for connection between the EndoButton and hamstring tendons: comparison with Ethibond sutures and Endobutton tape. J Biomed Mater Res $\underline{6}, 173-181$ 
Warden WH, Friedman R, Teresi LM, Jackson DW (1999): Magnetic resonance imaging of bioabsorbale polylactic acid interference screws during the first 2 years after anterior cruciate ligament reconstruction. Arthroscopy 15, 474-480

Weber W, Weber E: Mechanik der menschlichen Gehwerkzeuge; Springer Verlag, Berlin 1836

Weiler A, Hoffmann RF, Stahelin AC, Helling HJ, Sudkamp NP (2000): Biodegradable implants in sports medicine: the biological base. Arthroscopy 16, $305-321$

Wilson TC, Kantaras A, Atay A, Johnson DL (2004): Tunnel enlargement after anterior cruciate ligament surgery. Am J Sports Med 32, 543-549

Wolf RS, Lemak LJ (2002): Revision anterior cruciate ligament reconstruction surgery. J South Orthop Assoc 11, 25-32

Yoshiya S, Nagano M, Kurosaka M et al. (2000): Graft healing in the bone tunnel in anterior cruciate ligament reconstruction. Clin Orthop 376, 278-286

Zantop T, Weimann A, Schmidtko R, Herbort M, Raschke MJ, Petersen W (2006): Graft laceration and pullout strength of soft-tissue anterior cruciate ligament reconstruction: in vitro study comparing titanium, poly-d,l-lactide, and poly-d,l-lactide-tricalcium phosphate screws. Arthroscopy 22, 1204-1210

Zantop T, Herbort M, Raschke MJ, Fu FH, Petersen W (2007): The role of the anteromedial and posterolateral bundles of the anterior cruciate ligament in anterior tibial translation and internal rotation. Am J Sports Med 35, 223-227

Zimny ML, Schutte M, Dabezies E (1986): Mechanoreceptors in the human anterior cruciate ligament. Anat Rec 214, 204-209

Zuppinger H: Die aktive Flexion im unbelasteten Kniegelenk; Bergmann Verlag, Wiesbaden 1904 


\section{Veröffentlichungen}

Veröffentlichte Vorträge zur Dissertation:

Frosch KH, Sawallich T, Schütze G, Losch A, Floerkemeier T, Balcarek P, Walde T, Stürmer KM: Kernspintomographisches Abbau und Integrationsverhalten der neuartigen Interferenzschraube Milagro® ${ }^{\circledR}$ für den vorderen Kreuzbandersatz.

Deutscher Kongress für Orthopädie und Unfallchirurgie, Berlin 2007.

Teile dieser Arbeit wurden veröffentlicht in:

Frosch KH, Sawallich T, Schütze G, Losch A, Walde T, Balcarek P, Konietschke F, Stürmer KM (2009): Magnetic resonance imaging analysis of the bioabsorbable Milagro ${ }^{\circledR}$ interference screw for graft fixation in anterior cruciate ligament reconstruction. Strat Traum Limb Recon 4. $73-79$. 


\section{Abkürzungsverzeichnis}

\begin{tabular}{|c|c|}
\hline abs. & absolut \\
\hline Akn & Aktivitätsniveau \\
\hline AM & anterior-mediales \\
\hline AP & anterior-posterior \\
\hline BMI & Body-Mass-Index \\
\hline BRD & Bundesrepublik Deutschland \\
\hline BTB & Bone Tendon Bone \\
\hline bzw. & beziehungsweise \\
\hline ca. & circa \\
\hline $\mathrm{cm}$ & Zentimeter \\
\hline CT & Computertomographie \\
\hline DESS & double echo steady state \\
\hline$d_{g}$ & Schraubendurchmesser gemessen \\
\hline$d_{t}$ & Schraubendurchmesser tatsächlich \\
\hline et al. & et alii \\
\hline ICRS & International Cartilage Repair Society \\
\hline IKDC & International Knee Documentation Comitee \\
\hline internat. & international \\
\hline $\mathrm{kg}$ & Kilogramm \\
\hline km & Kilometer \\
\hline Konf.Interv. & Konfidenzintervall \\
\hline$I_{g}$ & Schraubenlänge gemessen \\
\hline $\mathrm{I}_{\mathrm{t}}$ & Schraubenlänge tatsächlich \\
\hline LCA & Ligament Cruciatum Anterius \\
\hline $\max$. & maximal \\
\hline mind. & mindestens \\
\hline Mm. & Musculi \\
\hline $\mathrm{mm}$ & Millimeter \\
\hline MMD & maximal manual drawer \\
\hline MRT & Magnet Resonanz Tomographie \\
\hline $\mathrm{N}$ & Newton \\
\hline neg. & negativ \\
\hline PDLLA & Poly-D lactic acid \\
\hline
\end{tabular}


PL

PGA

PLA

PLLA

PNF

pos.

SE

ST

Stdabw.

STG

TCP

teilw.

TFG

TSE

USA

$\mathrm{V}_{1}$

$\mathrm{V}_{2}$

VAS

VKB

vollst.

z.B.

posterior-laterales

Polyglycolid acid

Polylactid acid

Poly-L lactic acid

Propriozeptive Neuromuskuläre Fazilitation

positiv

spin echo

Semitendinosussehne

Standardabweichung

Semitendinosus- und Gracilissehne

Tri Calcium Phospat

teilweise

Tibiofemorales Gelenk

turbo spin echo

United States of America

Schraubenvolumen präoperativ

Schraubenvolumen postoperativ

Visuelle Analog Skala

vorderes Kreuzband

vollständig

zum Beispiel 
9. Abbildungs- und Tabellenverzeichnis

$\begin{array}{llll}\text { Abbildung 1: } & \text { S. } 11 & \text { Tabelle 1: } & \text { S.21 } \\ \text { Abbildung 2: } & \text { S. } 12 & \text { Tabelle 2: } & \text { S.21 } \\ \text { Abbildung 3 a/b: } & \text { S. } 12 & \text { Tabelle 3: } & \text { S.28 } \\ \text { Abbildung 4 a/b: } & \text { S.13 } & \text { Tabelle 4: } & \text { S. 30 } \\ \text { Abbildung 5: } & \text { S.13 } & \\ \text { Abbildung 6: } & \text { S.14 } & \\ \text { Abbildung 7: } & \text { S.17 } & \\ \text { Abbildung 8: } & \text { S.19 } & \\ \text { Abbildung 9: } & \text { S.22 } & \\ \text { Abbildung 10: } & \text { S.23 } & \\ \text { Abbildung 11: } & \text { S. } 24 & \\ \text { Abbildung 12: } & \text { S.24 } & \\ \text { Abbildung 13: } & \text { S. } 25 & \\ \text { Abbildung 14: } & \text { S.26 } & \\ \text { Abbildung 15: } & \text { S.26 } & \\ \text { Abbildung 16: } & \text { S.28 } & \\ \text { Abbildung 17: } & \text { S.29 } & \\ \text { Abbildung 18: } & \text { S.31 } & \\ \text { Abbildung 19a/b/c: } & \text { S.32 } & \\ \text { Abbildung 20 a/b/c: } & \text { S.33 } & \\ \text { Abbildung 21: } & \text { S.35 } & \\ \text { Abbildung 22 a/b/c: } & \text { S.36 } & \\ \text { Abbildung 23 a/b/c: } & \text { S.37 } & \\ \text { Abbildung 24 } & \text { S.38 } & \\ \end{array}$


10. Anhang: Scores

\section{$\underline{\text { Tegner Score }}$}

\section{Wettkampfsport (Fußball, national-internationale Elite)} Wettkampfsport

\begin{tabular}{|c|c|c|c|c|}
\hline \multirow{2}{*}{\multicolumn{3}{|c|}{ 它 }} & \multirow{2}{*}{\multicolumn{2}{|c|}{\begin{tabular}{l|l} 
Ist & prä OP
\end{tabular}}} \\
\hline & & & & \\
\hline \multicolumn{2}{|c|}{ Wettkampfsport (Fußball, national-internationale Elite) } & 10 & & \\
\hline \multicolumn{5}{|c|}{ Wettkampfsport } \\
\hline & Fußball, niedrigere Klassen & 9 & & \\
\hline & Eishockey & & & \\
\hline & Wrestling & & & \\
\hline & Gymnastik & & & \\
\hline \multicolumn{5}{|c|}{ Wettkampfsport } \\
\hline & Bandy & 8 & & \\
\hline & Squash / Badminton & & & \\
\hline & Leichtathletik (Sprünge) & & & \\
\hline & Skiabfahrt & & & \\
\hline \multicolumn{5}{|c|}{ Wettkampfsport } \\
\hline & Tennis & 7 & & \\
\hline & Leichtathletik (Lauf) & & & \\
\hline & Motocross / Speedway & & & \\
\hline & Handball & & & \\
\hline & Basketball & & & \\
\hline \multicolumn{5}{|l|}{ Breitensport } \\
\hline & Fußball & 7 & & \\
\hline & Badminton / Eishockey & & & \\
\hline & Leichtathletik (Sprünge) & & & \\
\hline & Marathonlauf / Orientierungslauf & & & \\
\hline \multirow{6}{*}{ Breitensport } & & 6 & & \\
\hline & Tennis / Badminton & & & \\
\hline & Handball & & & \\
\hline & Basketball & & & \\
\hline & Skifahren & & & \\
\hline & Jogging ( mind. $5 \times$ pro Woche) & & & \\
\hline \multirow{4}{*}{$\begin{array}{l}\text { Arbeit } \\
\text { Wettkampfspo }\end{array}$} & Schwerarbeit (Bauarbeiter) & 5 & & \\
\hline & & & & \\
\hline & Radfahren & 5 & & \\
\hline & Skilanglauf & & & \\
\hline Breitensport & Jogging auf unebenem Boden & 5 & & \\
\hline & (mind. $2 \times$ pro Woche) & & & \\
\hline \multirow[t]{2}{*}{ Arbeit } & mittelschwere Arbeit & 4 & & \\
\hline & (LKW-Fahrer) & & & \\
\hline \multirow[t]{3}{*}{ Breitensport } & Radfahren & 4 & & \\
\hline & Skilanglauf & & & \\
\hline & Jogging auf ebenem Boden & & & \\
\hline \multirow{4}{*}{$\begin{array}{l}\text { Arbeit } \\
\text { Wettkampf- un } \\
\text { Breitensport }\end{array}$} & leichte körperliche Arbeit & 3 & & \\
\hline & & & & \\
\hline & Schwimmen & & & \\
\hline & Laufen im Wald möglich & & & \\
\hline Arbeit & leichte Arbeit & 2 & & \\
\hline \multicolumn{5}{|c|}{ Gehen auf unebenem Grund möglich, jedoch nicht im Wald } \\
\hline Arbeit & Sitzende Tätigkeit & 1 & & \\
\hline \multicolumn{5}{|c|}{ Gehen auf unebenem Grund noch möglich } \\
\hline Berentet aufgr & n Knieproblemen & 0 & & \\
\hline
\end{tabular}




\section{Nachuntersuchung VKB-Plastik: Lysholm und Marshall Score}

\begin{tabular}{|c|c|c|c|c|}
\hline Kriterien & Lysholm & Ist & Marshall & \\
\hline Hinken & kein & 5 & Run in place & $\mathrm{Ja}=1$ \\
\hline \multirow[t]{2}{*}{5} & leicht oder periodisch & 3 & \multirow{6}{*}{$\begin{array}{l}\text { Einbeinsprung } \\
\text { mit Einschränkung } \\
\text { unmöglich } \\
\text { Entengang } \\
\text { mit Einschränkung }\end{array}$} & Nein $=0$ \\
\hline & deutlich & 0 & & 2 \\
\hline & & & & 1 \\
\hline Hilfsmittel & ohne & 5 & & 0 \\
\hline \multirow[t]{2}{*}{5} & Stock oder Gehhilfe & 3 & & 2 \\
\hline & Belastung unmöglich & 0 & & 1 \\
\hline \multicolumn{3}{|c|}{ Treppensteigen } & \multirow{5}{*}{ Schwierigkeit } & \\
\hline \multirow[t]{4}{*}{10} & keine Probleme & 10 & & \multirow{4}{*}{$\begin{aligned} J a & =0 \\
\text { Nein } & =1\end{aligned}$} \\
\hline & leichte Probleme & 6 & & \\
\hline & Stufe für Stufe & 2 & & \\
\hline & unmöglich & o & & \\
\hline \multirow{4}{*}{$\begin{array}{l}\text { Hocke } \\
5\end{array}$} & keine Probleme & 5 & \multirow[t]{4}{*}{ halbe Hocke } & $\mathrm{Ja}=1$ \\
\hline & leichte Probleme & 4 & & Nein $=0$ \\
\hline & nicht über $90^{\circ}$ & 2 & & $\mathrm{Ja}=1$ \\
\hline & unmöglich & 0 & & Nein $=0$ \\
\hline
\end{tabular}

\section{Gehen/ Laufen/ Springen}

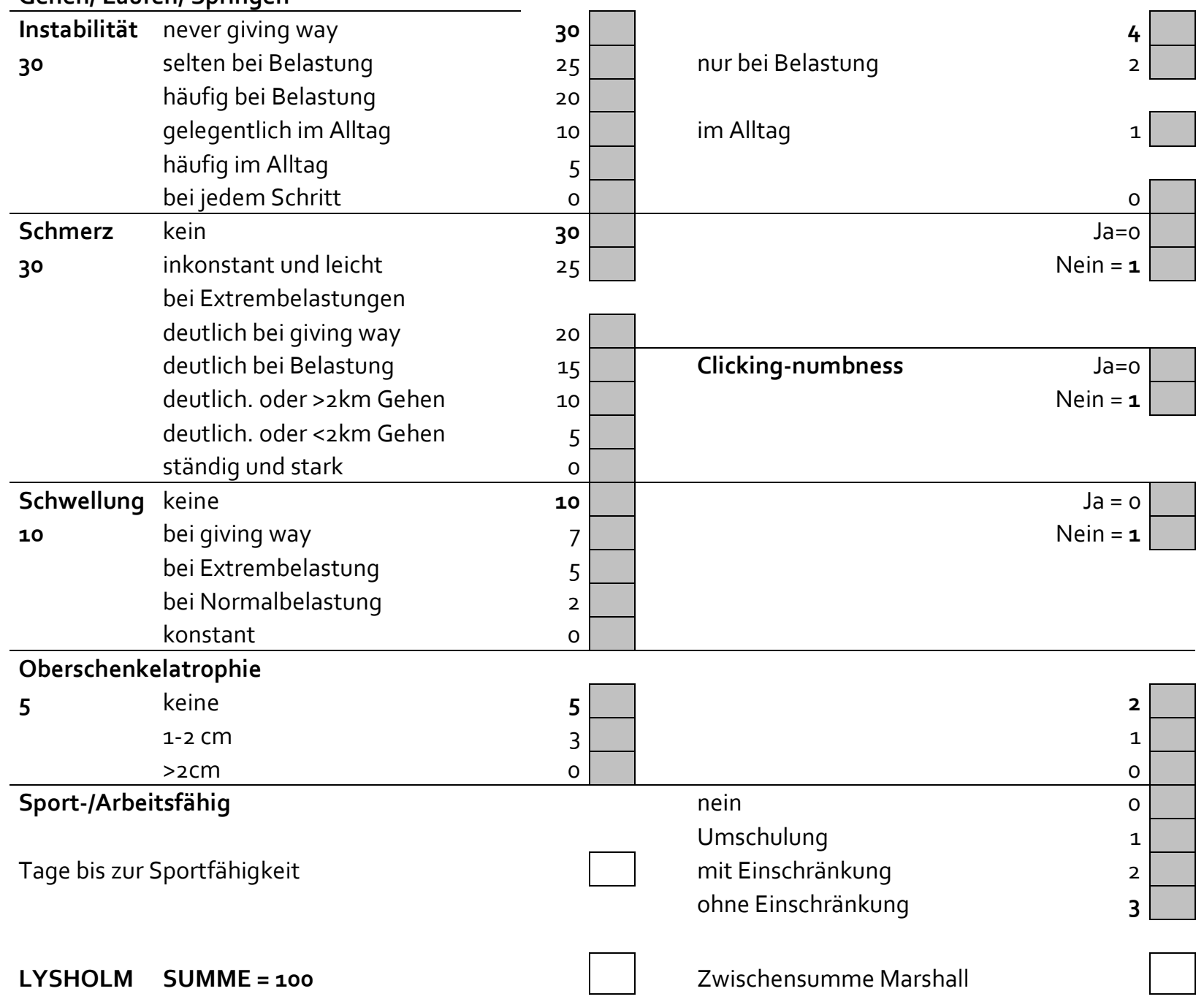




\section{Untersuchung des Kniegelenkes: Marshall Score}

Druckschmerz

Gelenkerguss

Weichteilschwellung

\section{Crepitation}

\begin{tabular}{|c|c|c|c|c|}
\hline & & & & \\
\hline $\mathrm{Ja}=\mathrm{o}$ & & & & \\
\hline \multicolumn{5}{|l|}{ Nein $=1$} \\
\hline $\mathrm{Ja}=0$ & 0 & gering & mäßig & stark \\
\hline \multicolumn{5}{|l|}{ Nein $=1$} \\
\hline $\mathrm{Ja}=0$ & 0 & gering & mäßig & stark \\
\hline \multicolumn{5}{|l|}{ Nein $=1$} \\
\hline \multicolumn{5}{|l|}{$J a=0$} \\
\hline Nein $=1$ & & & & \\
\hline
\end{tabular}

\section{Muskelkraft}

\section{normal}

abgeschwächte Flexion oder Extension abgeschwächte Flexion und Extension sehr schwach

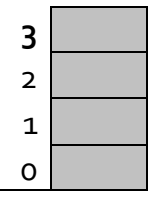

Bewegungsausmaß

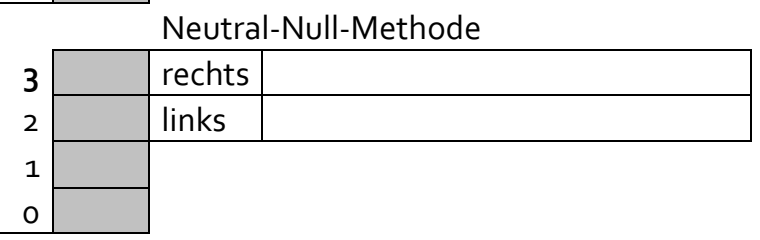

\section{Stabilität}

normal

limitierte Flexion oder Extension limitierte Flexion und Extension $<90^{\circ}$

LCL normal

leicht instabil in Flexion moderat instabil in Flexion instabil in Flexion und Extension grob instabil

\section{MCL normal}

leicht instabil in Flexion moderat instabil in Flexion instabil in Flexion und Extension grob instabil

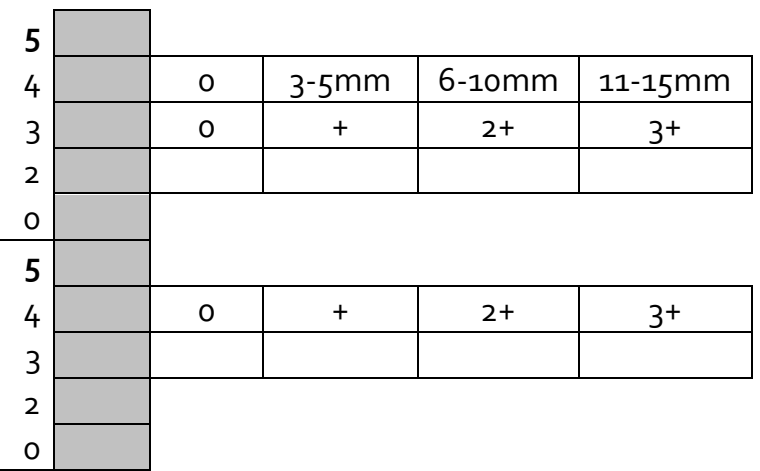

$\mathrm{ACL}$

\section{normal}

leicht instabil (Anschlag)

moderat instabil (Anschlag) instabil in Neutralposition instabil in Neutralposition u Rotation

PCL normal

leicht instabil (Anschlag) moderat instabil (Anschlag) instabil in Neutralposition instabil in Neutralposition und Rotation

\section{MARSHALL SUMME $=50$}

Steinmann I

Steinmann II

Appley

Payr

Zohlen

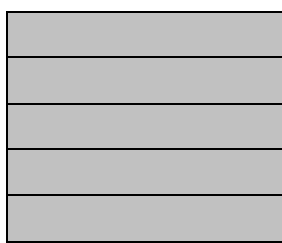

VAS SCHMERZ (0-10) :

$0=$ kein Schmerz
Lachman-Test

\begin{tabular}{|c|c|c|c|c|}
\hline 5 & 0 & + & $2+$ & $3^{+}$ \\
\hline 4 & & & & \\
\hline 3 & \multicolumn{4}{|c|}{ Vordere Schublade } \\
\hline 2 & 0 & + & $2+$ & $3^{+}$ \\
\hline 0 & & & & \\
\hline 5 & \multirow{2}{*}{\multicolumn{4}{|c|}{ Pivot Shift }} \\
\hline 4 & & & & \\
\hline 3 & 0 & + & $2+$ & $3^{+}$ \\
\hline \multicolumn{5}{|c|}{2} \\
\hline 0 & & & & \\
\hline
\end{tabular}

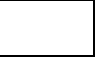

sonstiges:

VAS FUNKTION (10-0):

$10=$ gesund 


\section{IKDC-Score}

Aktivitätsniveau:

I = Aktivitäten mit belastender Rotation, Kontaktsportarten

II = Schwere körperliche Arbeit, Tennis, Skifahren

III = Leichtere körperliche Arbeit, Joggen, Springen

IV = Alltagsaktivitäten, sitzende Tätigkeit

Bewertung: A: normal B: fast normal C: abnormal D: stark abnormal

\section{Subjektive Beurteilungen durch Patient}

Wie funktioniert ihr Knie?

Wie beeinflusst Ihr Knie Ihre Aktivität auf einer Skala von o bis 3 ?
A:
B:
C:
D:
normal fast normal abnormal stark abnormal

1

2

3

2. Symptome (Fehlen von signifikanten Symptomen bei höchster vom Pat ausgeübten Aktivitätsniveau (Akn))

\begin{tabular}{|c|c|c|c|}
\hline Bis zu welchem Akn haben Sie keine Schmerzen? & I & II & III \\
\hline Bis zu welchem Akn haben Sie keine Schwellung? & I & II & III \\
\hline Bis zu welchem Akn haben sie kein teilw. Schnappen? & I & II & III \\
\hline Bis zu welchem Akn habe Sie kein vollständ. Schnappe & & II & III \\
\hline
\end{tabular}

\section{Bewegungsumfang: Flexion/Extension}

\section{Betroffene Seite : Gegenseite:}

Streckausfall (von anatomischer Nullstellung)

Flexionsausfall

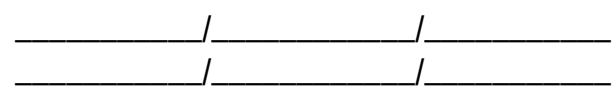

$\begin{array}{llll}<3^{\circ} & 3-5^{\circ} & 6-10^{\circ} & >10^{\circ} \\ 0-5^{\circ} & 6-15^{\circ} & 16-25^{\circ} & >25^{\circ}\end{array}$

\section{Untersuchung Bandapparat}

\section{Betroffene Seite:}

Lachman in $25^{\circ}$ Flexion

totale a.p. Translation in $70^{\circ}$ Flexion

Hinterer Durchhang in $70^{\circ}$ Flexion

med. Gelenköffnung (Valgusrotation)

lat. Gelenköffnung (Varusrotation)

Pivot Shift

Reversed Pivot Shift neg.

$\begin{array}{llll}\text { 0-2mm } & 3-5 \mathrm{~mm} & 6-10 \mathrm{~mm} & >10 \mathrm{~mm} \\ \text { 0-2mm } & 3-5 \mathrm{~mm} & 6-10 \mathrm{~mm} & >10 \mathrm{~mm} \\ \text { 0-2mm } & 3-5 \mathrm{~mm} & 6-10 \mathrm{~mm} & >10 \mathrm{~mm} \\ \text { 0-2mm } & 3-5 \mathrm{~mm} & 6-10 \mathrm{~mm} & >10 \mathrm{~mm} \\ \text { 0-2mm } & 3-5 \mathrm{~mm} & 6-10 \mathrm{~mm} & >10 \mathrm{~mm} \\ \text { neg. } & +{ }^{\prime} & ++ & +++ \\ \text { neg. } & \text { gering } & \text { markant. } & \text { pos. }\end{array}$




\section{Kompartimentale Befunde}

\section{Betroffene Seite}

Patellofemorale Krepitation

$\begin{array}{llrl}\text { kein } & \text { mäßig } & \text { schmerzhaft } & \text { stark } \\ \text { kein } & \text { mäßig } & \text { schmerzhaft } & \text { stark } \\ \text { kein } & \text { mäßig } & \text { schmerzhaft } & \text { stark }\end{array}$

\section{Gesunde Seite}

Patellofemorale Krepitation

kein mäßig schmerzhaft stark

Krepitation mediales Kompartiment

kein mäßig schmerzhaft stark

Krepitation laterales Kompartiment

kein mäßig schmerzhaft stark

\section{Symptome bei Transplantatentnahmestelle}

Druckschmerz, Irritation, Gefühlsstörung

kein gering mäßig stark

\section{Röngenbefunde (im Rahmen der Nachuntersuchung nicht erfolgt)}

\section{Betroffene Seite}

Patellofemoraler Gelenkspalt

Medialer Gelenkspalt

$\begin{array}{llll}\text { normal } & >4 \mathrm{~mm} & 2-4 \mathrm{~mm} & <2 \mathrm{~mm} \\ \text { normal } & >4 \mathrm{~mm} & 2-4 \mathrm{~mm} & <2 \mathrm{~mm} \\ \text { normal } & >4 \mathrm{~mm} & 2-4 \mathrm{~mm} & <2 \mathrm{~mm}\end{array}$

Lateraler Gelenkspalt

normal

$4 \mathrm{~mm}$

\section{Gesunde Seite}

Patellofemoraler Gelenkspalt

$\begin{array}{llll}\text { normal } & >4 \mathrm{~mm} & 2-4 \mathrm{~mm} & <2 \mathrm{~mm} \\ \text { normal } & >4 \mathrm{~mm} & 2-4 \mathrm{~mm} & <2 \mathrm{~mm} \\ \text { normal } & >4 \mathrm{~mm} & 2-4 \mathrm{~mm} & <2 \mathrm{~mm}\end{array}$

Lateraler Gelenkspalt

normal

$>4 \mathrm{~mm}$

$<2 \mathrm{~mm}$

\section{Funktioneller Test}

Sprung gesunde

Seite $\mathrm{cm}$

Sprung betroffene

Seite $\mathrm{cm}$

$90-100 \% \quad 76-90 \% \quad 50-75 \% \quad<5$




\section{Danksagung}

Ich danke Herrn Professor Dr. K.M. Stürmer, dem Leiter der Abteilung für Unfallchirurgie, Plastische und Wiederherstellungschirurgie, in dessen Abteilung ich diese Arbeit erstellen durfte.

Mein besonderer Dank gilt Herrn Professor Dr. K.-H. Frosch für die freundliche Überlassung des Themas, die fachliche Betreuung sowie die kontinuierliche Unterstützung und Hilfsbereitschaft bei der Durchführung dieser Dissertationsarbeit.

Herrn G. Schütze danke ich für die Unterstützung bei der Durchführung und Auswertung der kernspintomographischen Messreihe.

Für die Hilfe bei der statistischen Auswertung möchte ich Herrn F. Konietschke danken. 


\section{Lebenslauf}

Am 8. Juni 1977 wurde ich, Tobias Sawallich, als Sohn von Sieglinde Sawallich und Elmar Sawallich in Albstadt-Ebingen geboren.

Von 1984 bis 1987 besuchte ich die Grundschule in Winterlingen und anschließend bis 1988 die Grundschule in Lomersheim. Von 1988 an besuchte ich das Theodor-Heuss-Gymnasium in Mühlacker, an dem ich 1997 die Allgemeine Hochschulreife erlangte.

Von 1997 bis 1998 absolvierte ich meinen Zivildienst im Krankenhaus Mühlacker. Im Oktober 1998 begann ich das Studium der Wirtschaftspädagogik an der Universität Hohenheim und legte im März 2001 die Vordiplom-Prüfung ab. Anschließend war ich in der EDV-Abteilung bei der Firma Behr GmbH $\&$ Co in Stuttgart tätig und unternahm eine Work and Travel Reise nach Neuseeland.

Im Februar 2002 begann ich das Studium der Humanmedizin an der Universität in Graz und wechselte im Oktober 2002 an die Georg-August-Universität nach Göttingen. Dort legte ich im Dezember 2008 die Ärztliche Prüfung ab. Seit dem Februar 2009 bin ich als Assistenzarzt in der Chirurgie in Herzberg am Harz tätig. 